NIST Special Publication 500-305

\title{
Guidance for Evaluating Contactless Fingerprint Acquisition Devices
}

\author{
John Libert \\ John Grantham \\ Bruce Bandini \\ Stephen Wood \\ Michael Garris \\ Kenneth Ko \\ Fred Byers \\ Craig Watson
}

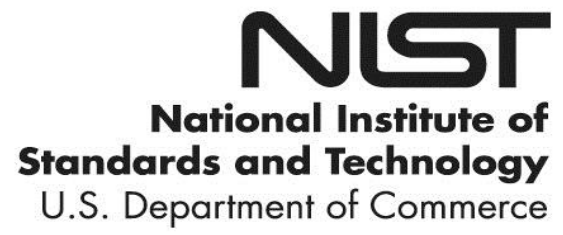


NIST Special Publication 500-305

\title{
Guidance for Evaluating Contactless Fingerprint Acquisition Devices
}

\author{
John Libert \\ Bruce Bandini \\ Stephen Wood \\ Michael Garris \\ Kenneth Ko \\ Fred Byers \\ Craig Watson \\ Information Access Division \\ Information Technology Laboratory \\ John Grantham \\ Systems Plus, Inc. \\ Rockville, $M D$
}

This publication is available free of charge from:

https://doi.org/10.6028/NIST.SP.500-305

July 2018

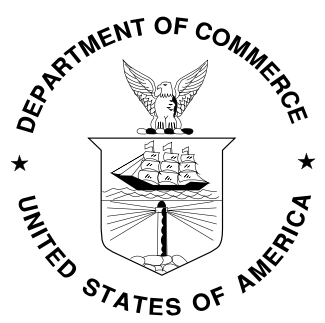

U.S. Department of Commerce Wilbur L. Ross, Jr., Secretary

National Institute of Standards and Technology Walter Copan, NIST Director and Under Secretary of Commerce for Standards and Technology 


\section{ACKNOWLEDGEMENTS}

We would like to acknowledge and thank the Federal Bureau of Investigation's (FBI) Criminal Justice Information Services (CJIS) Division as well as other key partners at the FBI who provided support to National Institute of Standards and Technology (NIST) in the development of this research in support of a Guidance for Evaluating Contactless Fingerprint Acquisition Devices.

\section{DisCLAIMER}

Certain commercial entities, equipment, or materials may be identified in this document to describe an experimental procedure or concept adequately. Such identification is not intended to imply recommendation or endorsement by the National Institute of Standards and Technology, nor is it intended to imply that the entities, materials, or equipment are necessarily the best available for the purpose.

The contactless devices used to collect data appearing in this report are under development. The analyses presented herein reflect the state of these devices at the time the data were collected and are not reflective of current or potential future performance. The analysis of the small samples of fingerprint images are intended only to serve as the medium by which to examine and demonstrate the behavior of candidate measurements and analysis procedures being developed by this NIST project.

The National Institute of Standards and Technology Human Subjects Protection Office reviewed the protocol for this project and determined it meets the criteria for exempt human subjects research as defined in Department of Commerce Regulations, 15 CFR 27, also known as the Common Rule for the Protection of Human Subjects (45 CFR 46, Subpart A).

National Institute of Standards and Technology Special Publication 500-305 Natl. Inst. Stand. Technol. Spec. Publ. 500-305, 76 pages (July 2018) 
VERSION HISTORY

\begin{tabular}{|l|l|}
\hline Date & Activity \\
\hline & \\
\hline & \\
\hline & \\
\hline
\end{tabular}




\begin{abstract}
This document details efforts undertaken by the National Institute of Standards and Technology (NIST) to develop measurements and a protocol for the evaluation of contactless (touchless) fingerprint acquisition devices. Contactless fingerprint capture differs fundamentally from legacy contact fingerprinting methods and poses novel problems for image quality evaluation and challenges relative to interoperability with contact fingerprints that populate large repositories maintained by law enforcement and Federal Government organizations. For contact acquisition, the fingerprint impression is a first-order transfer of the 3D friction ridge structure to the recording surface. The third dimension of the curved finger surface is effectively removed by pressure against the planar recording surface. The 3D topography of the ridges and furrows are transferred with low ambiguity to the recording surface as dark ridges (points of contact) and lighter furrows (lesser or no contact). Contactless images by comparison, in most cases, are third-order renderings of an original photographic representation, itself a 2D optical projection of the 3D structure of the finger. The appearance of this projection is subject to variability as low- or moderately-controlled lighting interacts with the 3D geometry of the finger, the friction ridge structure superimposed on the finger, and the geometry of the presentation of the finger to the contactless device. The photograph must then be subjected to various image processing methods to infer the ridge structure for rendering as a fingerprint similar in appearance to legacy contact captures. The rendering process is the source of numerous errors relative to contact captures. Despite problems with image quality, this early study finds contactless fingerprints of the devices examined to be useable in some applications, with qualifications, including one-to-many matching against small databases. Contactless users should expect larger error rates with machine matching and difficulty with any forensic applications such as latent matching, or support of courtroom testimony.
\end{abstract}

\title{
KEYWORDS
}

contactless fingerprint device; fingerprint matching; fingerprint sensors; image; registration; minutiae correspondence 


\section{EXECUTIVE SUMMARY}

With legacy contact methods, fingerprints are captured via a direct impression of friction ridge skin onto a recording medium. With early methods, ink-coated fingers were pressed or rolled onto standard cardstock. More recently, with advances in computer processing and digital storage technology, optical scanning of the inked card images has enabled the beginnings of automated fingerprint matching and the efficiencies of digital storage, retrieval, and electronic transmission of fingerprint records. Well into the "digital age", the scanning of inked impressions from paper largely gave way to direct digital capture of the friction ridge patterns via a variety of optical and electronic sensors, all involving contact of the finger to the recording device. Despite differences among the fingerprint impressions due to variations in pressure of the contact or variations in the digital representations among multiple samples of a friction ridge pattern, the constant is that with contact fingerprinting, the dark and light variation in the print is a function of the degree of contact with the topography of the friction ridge surface. The local topographic highs leave the darkest features and the topographic lows the lightest, in the most common "brightfield" case. The reverse is the case in the less common "darkfield" case. In either case, with contact devices, fingerprint impression is a first-order representation of the friction ridge topography.

Contactless fingerprinting offers many attractions. The fact that no contact is required allays concerns over hygiene for applications for which frequent cleaning of a device is inconvenient; Most contactless devices can be used with limited supervision or interaction with a trained technician; Applications installed on smartphones offer unprecedented opportunities for mobile capture for law enforcement, defense, and border security, as well as personal identity verification for a wide variety of applications. Despite these desirable features that currently drive the demand for contactless fingerprinting, the key element lacking with contactless capture is the direct relationship to the friction ridge structure such devices attempt to represent. The photographic record of the 3D friction ridge topography is at best a second-order representation subject to the vagaries of illumination reflecting from a complex surface. Any algorithm applied to the photograph to transform the photograph into a representation closer in appearance to a contact impression adds a third-order to the representation. The indirect capture of friction ridge detail poses interoperability challenges for contactless fingerprinting that the present paper attempts to characterize.

In Section 2, we summarize the operational characteristics of some contactless devices the NIST team has examined. All involve photographic capture of the finger image to some degree. In Section 3, we discuss some of the main quality attributes included in the current quality specification for Personal Identity Verification (PIV) and relevance to fingerprint acquisition from 3D surfaces and possible modifications for 3D application. In Section 4 we describe the approach taken toward evaluating image quality mainly in the context of interoperability with contact mates starting with registration of the contactless fingerprint images with contact mates. 
Section 5 describes the specific measurements applied to individual images as well as to overlapping regions of contactless fingerprints and their contact mates. Regarding interoperability between contactless and contact, we hoped to establish a baseline by which to understand strengths and weaknesses of the contactless technology relative to legacy fingerprint capture methods - to put observed differences between contactless and contact into perspective relative to contact device interoperability. Also, in Section 5, we provide results of some early single finger matching tests. We note that considerably more matcher testing is planned for the next phase of this effort, using larger data sets to better support multiple finger (subject) matching rather than the single finger tests.

In Section 6 we describe some image anomalies that might inhibit the interoperability of contactless with contact fingerprints. Polarity (contrast) reversal presents significant problems for forensic application of contactless fingerprints. The polarity reversals will adversely impact the ability of examiners to correlate features of contactless prints with contact exemplars. Regions of the finger photograph in poor focus, shadow, or high reflection present insufficient detail for rendering of the ridge structure causing, at least, loss of detail or, at worst, the introduction of false detail that generate false minutiae upon characterization of the rendered contactless print. Skin irregularities such as wrinkles or scars that are typically flattened with contact capture are so emphasized by lighting effects as to obscure ridge detail. Yet we find some success with automated matching, though with higher error than with contact-to-contact comparisons.

In Section 7, we present examples of test artifacts under development for contactless device testing. Devices include both laser-engraved etched fingerprint and 3D geometric patterns designed to test for various performance anomalies. Ultimately, these or other suitable artifacts might be used for testing devices and made available to manufacturers for calibration and testing of their fingerprint capture systems. Some of the artifacts pose rendering difficulties for some contactless devices for which a friction ridge pattern provides information for scaling the output image to achieve the 500 ppi sample rate. Artifacts that present smooth regions pose further rendering difficulties for algorithms that rely on ridge flow orientation by which to guide ridge reconstruction. Procedures by which to evaluate device responses to the artifacts remain under development. 


\section{TABLE OF CONTENTS}

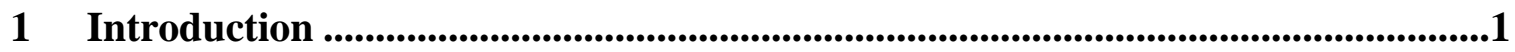

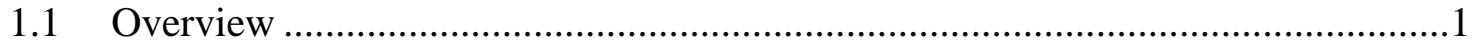

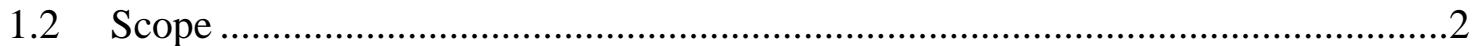

2 The Nature of Contactless Devices......................................................................3

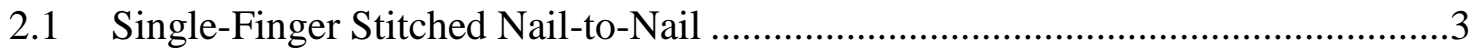

2.2 Hand-In-Motion Texture Image and Structure Light ...............................................

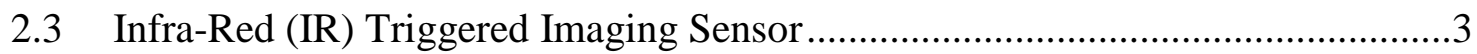

2.4 Manual or Focus Triggered Photographic Capture ……………..........................

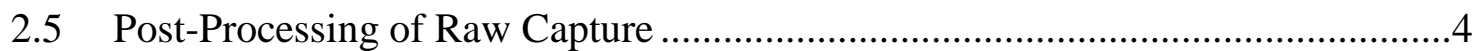

3 Consideration of PIV Basic Requirements.....................................................................5

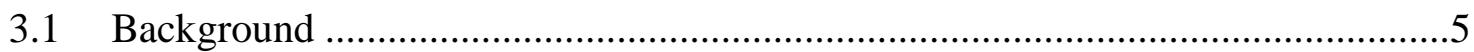

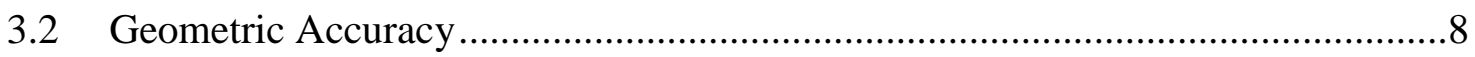

3.3 Spatial Frequency Response......................................................................

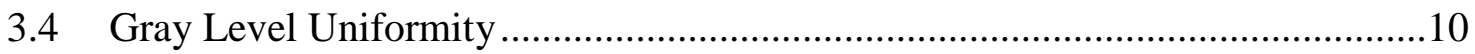

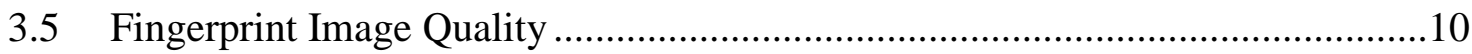

4 Image Quality and Interoperability Metrics............................................................11

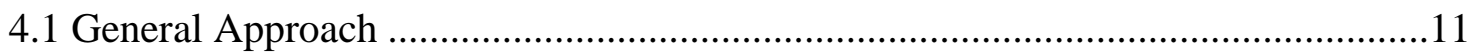

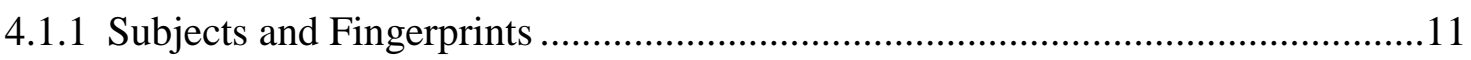

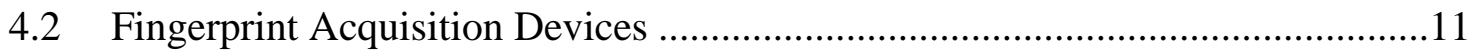

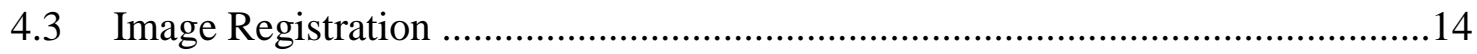

4.4 Control Point Selection and Transform Refinement ..............................................14

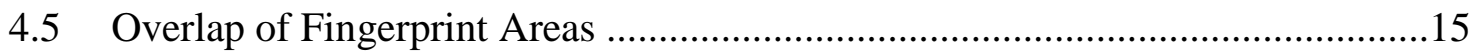

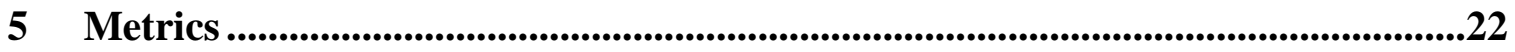

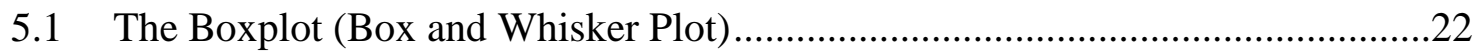

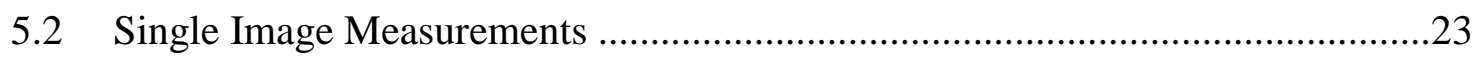

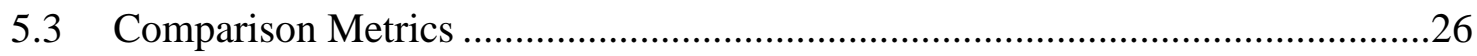

5.4 Discussion of Quality and Interoperability …………………........................

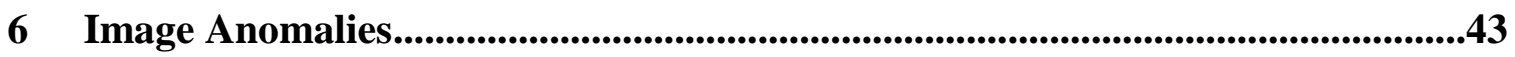

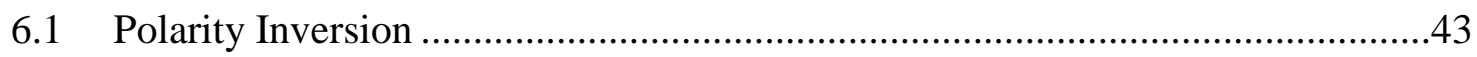

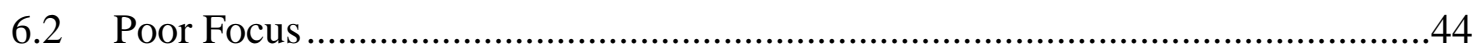

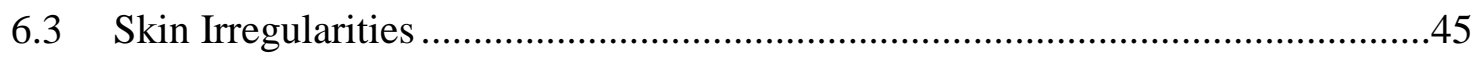

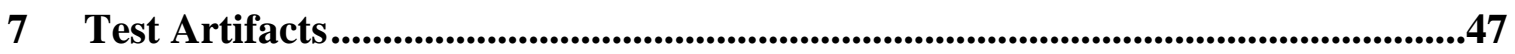




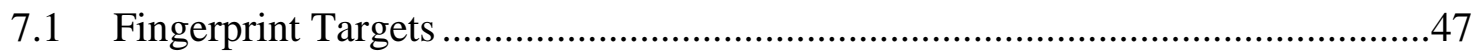

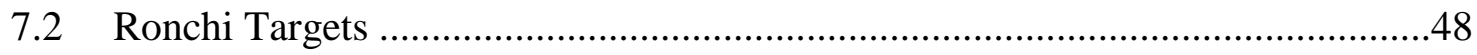

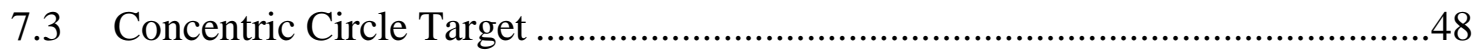

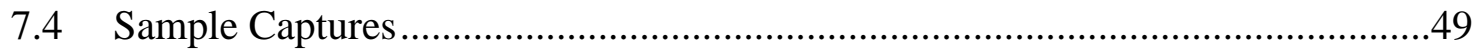

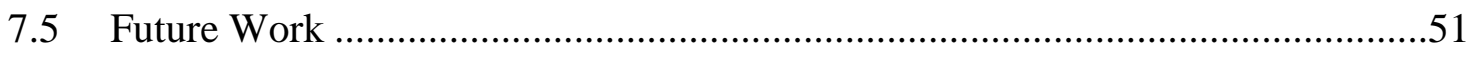

8 References...................................................................................................................52

9 Appendix A Minutiae Extraction and Correspondence .....................................54

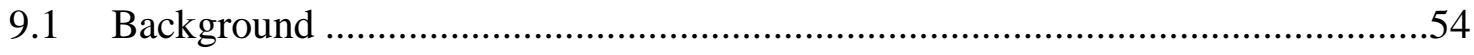

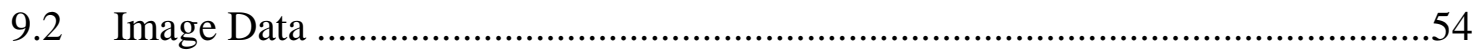

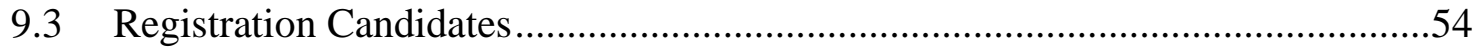

10 Appendix B Matcher Testing.........................................................................56

11 Appendix C - Fabrication of Test Artifacts ............................................................58

11.1 Material Specification and Artifact Dimensions ............................................58

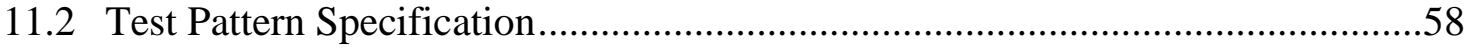

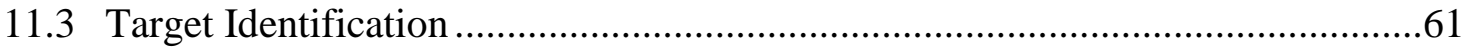

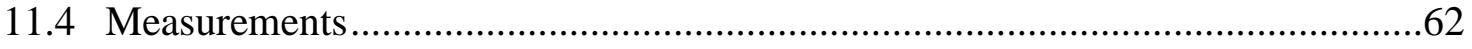

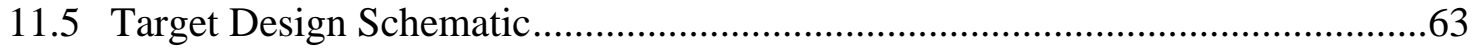




\section{LIST OF TABLES}

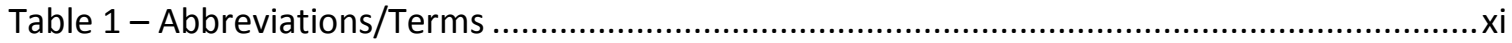

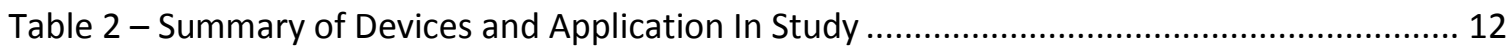

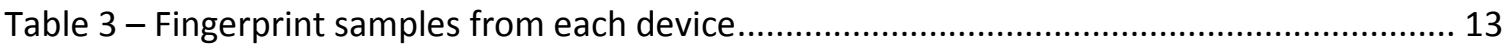

Table 4 - Scale variability across contact capture technologies .................................................. 27

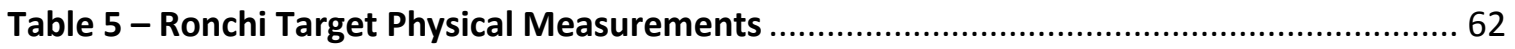

\section{LIST OF FIGURES}

Figure 1 - Image of a portion of one of the target artifacts. Most of the surface of this artifact is polished smooth, yet the rendering algorithm introduces false texture or "feature noise". . 8

Figure $2-1.0 \mathrm{cy} / \mathrm{mm}$ Ronchi Ruling Target (enlarged example of $15.5 \times 19 \mathrm{~mm}$ original) ........... 9

Figure 3 - Overlay of registered fingerprint image pair showing control points (marked with triangles) generating the affine transform that minimized displacement distance among candidate minutiae.

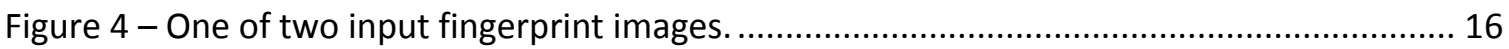

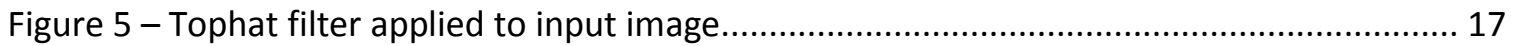

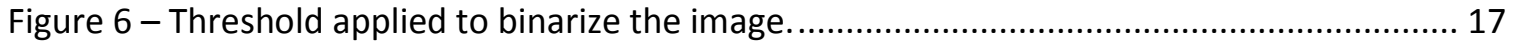

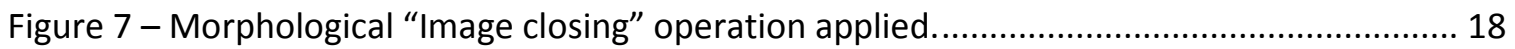

Figure 8 - Having applied the above procedure to the two registered fingerprint images, we sum the binary images yielding an image of values zero where no fingerprint is found (dark blue), one for non-overlapping finger region (turquoise), and the overlapping region summing to two (yellow).

Figure 9 - Locating coordinates designating the smallest rectangle enclosing the non-zero values in $\mathrm{B}_{\mathrm{C}}$, gives us cropping coordinates by which to extract the overlapping regions from the original registered image pair.

Figure 10 - Overlapping regions of a registered fingerprint pair............................................... 21

Figure 11 - Relationship between the boxplot of normally distributed data compared to the standard normal distribution for illustrative purposes. (Graphic from [WMC]).................... 22

Figure 12 - Image entropy for fingerprint images of each of the devices, both contactless and contact used in the study.

Figure 13 - BSNR values for fingerprint acquisitions from three contactless fingerprint devices and for six contact devices.

Figure 14 - NFIQ 2.0 scores for acquisitions of each of 3 contactless devices and each of the two acquisitions from the control FTIR contact device.

Figure 15 - We multiply scale factor by 500 ppi to get familiar sample rates for fingerprints. For both PIV and Appendix F, we expect images at 500 ppi. The PIV specification allows values between $510 \mathrm{ppi}$ and $490 \mathrm{ppi}$, and the more stringent Appendix $\mathrm{F}$ allows sample rates between 505 ppi and 495 ppi. (Dashed lines indicate exclusion of extreme outliers.) .......... 28

Figure 16 - Overlap between contact fingerprint and corresponding contactless acquisition or between the mated contact acquisitions. 29 
Figure 17-SIVV spectra of the two (synthetic) fingerprint impressions shown above. Peak location corresponds to spatial frequency of ridge pattern. Applying a small degree of low-pass filtering to img1 reduces power in the high frequencies observed in its processed mate, img2.

30

Figure 18 - Root Mean Squared Difference of the spectra of contactless to contact and various contact to contact comparisons. Lower values are better. (Dashed line indicates exclusion of extreme outliers.).

Figure 19 - SIVV correlation between spectra of corresponding contactless and contact exemplars; between two encounters from $\mathrm{C} 1$, and between different contact devices. ..... 32

Figure 20 - Synthetic fingerprint showing vector field of estimated ridge orientations.............. 33

Figure 21 - Distribution of correlation of ridge orientation maps estimated for overlapping regions of fingerprints under comparison, contactless devices TA, TB, TBrp, and TM against contact exemplars and for mated contact impressions.

Figure 22 - Distributions of CWSSIM comparisons for contactless to contact and contact to contact devices. The CWSSIM is sensitive to differences in both contrast and local structure of images, hence the highest values for $\mathrm{C} 1$, where both images are acquired using the same FTIR device.

35

Figure 23 - Numbers of corresponding minutiae for comparison of each sensor capture with contact control impressions as determined by the state-of-the-art fingerprint feature detector.

Figure 24 - Distributions of mean Euclidean distance between positions of corresponding minutiae on spatially registered fingerprint impressions captured by devices compared to control contact capture. Distance is computed for all corresponding minutiae after application of the optimized transform.

Figure 25 - Distributions of matcher similarity scores of device captures and contact control impressions. A distribution of 5096 non-mate scores is included for reference (see far right).

Figure 26 - Receiver Operating Characteristic (ROC) graph for the small matcher test showing the True Match Rate as a function of False Match Rate.

Figure 27 - As designated by the red ellipses, note the three ridge endings in the photograph (left) captured by a contactless device and the corresponding features in the livescan capture (center). In the grayscale rendering of the contactless capture (right), the ridge endings become bifurcations (furrow endings). The delta feature designated by green ellipses is formed by ridges in the photo and livescan images, but by a furrow, incorrectly toned dark in the contactless print.

Figure 28 - Grayscale inversion of the original photograph depicted in Figure 27 illustrating that the furrows have been misrepresented as ridges giving us bifurcations rather than ridge endings.

Figure 29 - Contactless photograph (left) shows a portion of a photographic capture of a finger. The contact capture of the friction ridge region (center) is crisply rendered to the point of showing level 3 features such as pores. The poor focus has inhibited rendering of the output grayscale fingerprint as seen in the rightmost image. .45

Figure 30 - The contact capture on the left exhibits skin irregularities that interrupt the ridge structure. These features are visible in the photographic capture (center) as grooves, with the 3D relief emphasized by the showing proximal to the illumination source and highlights on the distal sides of the grooves receiving direct lighting. The grayscale rendering on the right shows the grooves becoming major structural features that compete in significant way with 
the friction ridge structure. Polarity reversal is also evident as the illuminated flanks of the grooves are rendered dark and the shadows rendered bright......................................... 46

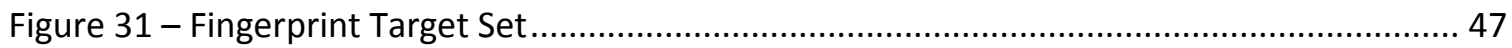

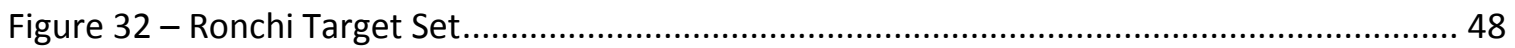

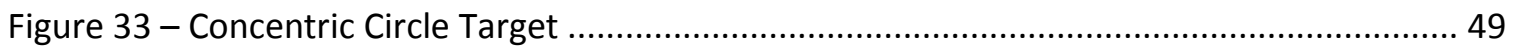

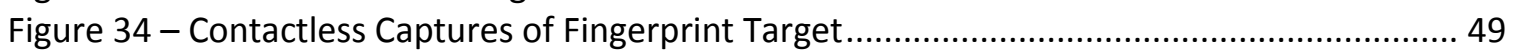

Figure 35 - Contactless Captures of Vertical Ronchi Target .................................................... 50

Figure 36 - Contactless Captures of Concentric Circle Target .................................................. 50

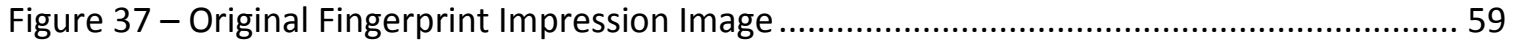

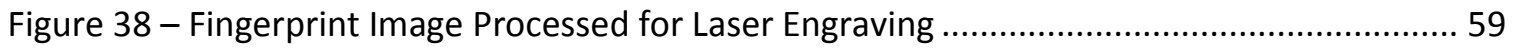

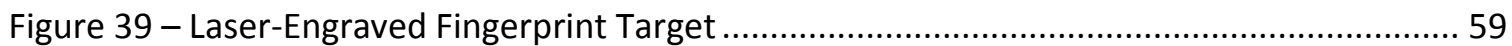

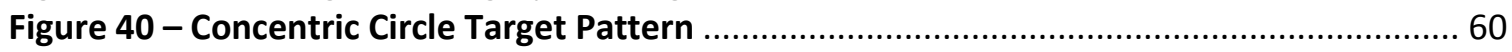

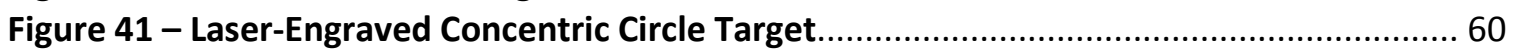

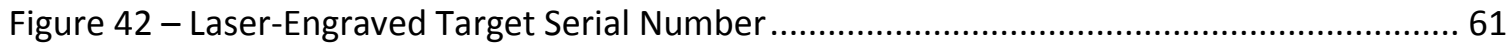

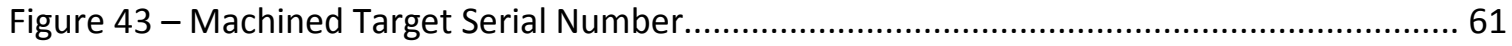

Figure 44 - Design schematic for fabrication of targets from 1" $(25.4 \mathrm{~cm})$ acrylic rods. Units shown

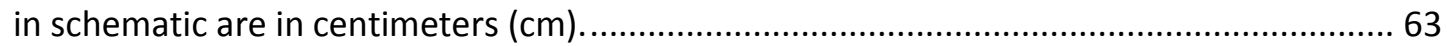




\section{TERMS AND DEFINITIONS}

Table 1 - Abbreviations/Terms

\begin{tabular}{|l|l|}
\hline Appendix F & FBI fingerprint image quality specification (see reference [EBTS]) \\
\hline bpp & Bits per pixel \\
\hline CJIS & Criminal Justice Information Services Division \\
\hline CODEC & Encoder and Decoder \\
\hline FBI & Federal Bureau of Investigation \\
\hline FTIR & Frustrated Total Internal Reflection \\
\hline IAI & International Association for Identification \\
\hline ITL & Information Technology Laboratory \\
\hline LED & Light Emitting Diode \\
\hline NBIS & NIST Biometric Image Software \\
\hline NIJ & National Institute of Justice \\
\hline NIST & National Institute of Standards and Technology \\
\hline PGM & Portable Graymap (image) Format \\
\hline PIV & Personal Identity Verification (see PIV specification in References) \\
\hline ppi & Pixels per inch (the customary unit of sampling for digital fingerprints) \\
\hline ppmm & Pixels per millimeter \\
\hline SIVV & Spectral Image Validation/Verification Metric \\
\hline
\end{tabular}




\section{Introduction}

\subsection{Overview}

Contactless acquisition of fingerprints presents a fundamental departure from legacy capture technologies. The friction ridge surface that comprises the fingerprint is a threedimensional topography superimposed upon the three-dimensional, curved surface of a finger. Yet rendered fingerprint impressions, themselves, are two-dimensional representations of the three-dimensional structure. Currently, there are no 3D representations of fingerprints as point-clouds ( $\mathrm{x}, \mathrm{y}, \mathrm{z}$ coordinates) that sufficiently resolve ridge topography on the finger surface and that demonstrate compatibility with legacy fingerprint databases.

For contact acquisition, the third dimension is effectively removed by the contact with the surface of the recording medium, be that paper, the platen of an FTIR optical device, or other flat surface used in capacitance and ultrasonic devices. Even though the finger surface is curved, it is flattened by deformation of the finger with pressure against the capture surface. The height of ridges is acknowledged only to the extent that topographic high points (i.e., ridges), are recorded and lows (i.e., furrows) are not. For a rolled acquisition, the three-dimensional structure of the finger is unwrapped to a plane by rolling the finger across the capture surface. For plain (flat) capture, the finger pad is flattened against the capture surface. Despite various forms and degrees of distortion possible with contact capture, the outstanding characteristic of legacy fingerprint acquisition is that ridges are unambiguously recorded by the contact process itself. The representation of the friction ridge surface might suffer certain defects due to insufficient pressure, too much pressure, over- or under-inking, sliding, etc., but where the ridge appears on the paper or in the digital image will be close to the truth.

In inked fingerprint capture, the print on the card is a representation of the friction ridge surface. When the card is scanned, the digital image is a representation of the inked impression - a second-order representation of the ridge structure of the finger surface. For FTIR devices, we have a digital image as a first-order representation of the friction ridge surface. In this regard, it is noted that for ink-on-card fingerprints, most defects that affect quality occur before the digitization process, i.e., at the point that the inked impression is recorded on the paper. Additional error could result from conversion of the inked impression to digital form via optical scanning, though for inked impressions, the quality is mainly determined before optical scanning is brought to bear.

For contactless acquisition, we have a series of representations with a departure from legacy capture - the loss of clear discrimination of topographic highs and lows. For those contactless devices examined by NIST, all start with an optical representation of an illuminated finger surface - a photographic image of some sort, itself a projection of the 3D structure onto a 2D surface. The interaction of light with the friction ridge surface lacks the unambiguous relationship between light and darks of the fingerprint with the topography of the friction ridge surface. With contactless capture, we have a secondorder representation created from the photographic source by application of unspecified image processing procedures to convert the photographic representation into a grayscale 
or binary image that more closely resembles that of a legacy capture. The accuracy of this second-order representation (model) of the friction ridge topography is affected by the defects of the photograph such as blurring due to motion or poor focus, and the vagaries of lighting.

\subsection{Scope}

The present document should be considered only a description of our exploratory study of the performance of contactless devices and their interoperability with contact fingerprint acquisition devices. Image data from the few contactless devices covered by this phase of our research enabled the testing of metrics and procedures that might prove useful in an eventual protocol for conformance testing of such devices. The devices included have continued to be refined, and new devices have been proposed since the beginning of this research project. Hence, contactless device performance relative to the various measurements summarized herein, provide only a coarse view of contactless performance relative to that of contact devices. 


\section{The Nature of Contactless Devices ${ }^{1}$}

Contactless devices, by definition, acquire fingerprints either individually or in multiples without the friction ridge skin contacting the device. Other parts of the hand, in some cases, may contact the device either to ensure proper positioning or stability during capture. Beyond the attribute of being contactless, different approaches have been taken toward capture of the fingerprint image ${ }^{2}$.

\subsection{Single-Finger Stitched Nail-to-Nail}

The contactless fingerprint development derived from a call for rapid capture nail-to-nail fingerprint acquisition [NIJ2]. Responding to this challenge, several companies developed schemes by which a finger would be illuminated from multiple directions and imaged simultaneously with multiple imaging sensors. The multiple images are then "stitched" together along common boundaries much as with the construction of a panoramic image from multiple frames shot with a modern digital camera. It is possible that some developers attempt to compensate for the curvature of the finger by projecting the images onto a cylinder before final "unwrapping" into the rolled equivalent that is the objective output of the process.

\subsection{Hand-In-Motion Texture Image and Structure Light}

One device requires, for proper acquisition, that multiple fingers, single finger, or thumbs are swept through the capture volume. In operational mode ${ }^{3}$, the motion is required for capture. The device uses multiple color illuminants. The texture image of the fingerprint is recorded by the green illuminant. The red illuminant projects a set of bands onto the target, the structured light pattern that is used to estimate the shape of the surface onto which the friction ridge texture is overlain. The $3 \mathrm{D}$ shape is projected onto a plane and the estimated shape parameters are used to "unwrap" the texture image to remap pixels on the sides of the print to their inferred correct position on the planar surface of the rendered fingerprint image.

\subsection{Infra-Red (IR) Triggered Imaging Sensor}

This IR-triggered device is designed to record images of single or multiple fingers, the presence of which in the capture volume is signaled by a near infra-red illuminant and sensor. The device makes no attempt to apply geometric correction to the image, so the image is essentially a frontal image of the fingers, complete with the normal spatial distortion along the margins. In some cases, it is possible that some attempt is made to "stretch" the ridge pattern toward achieving more uniform ridge frequency. Illumination is provided by LED flash.

\footnotetext{
${ }^{1}$ In this report, the term, "device", always includes hardware and the software used in acquisition and processing of a fingerprint image.

2 The contactless devices used to collect data appearing in this report are under development. The analyses presented herein reflect the state of these devices at the time the data were collected and are not reflective of current or potential future performance. The analysis of the small samples of fingerprint images are intended only to serve as the medium by which to examine and demonstrate the behavior of candidate measurements and analysis procedures being developed by this NIST project.

3 "Operational mode" implies that all device functions are active as they would be in normal usage of the device.
} 


\subsection{Manual or Focus Triggered Photographic Capture}

Implemented on mobile devices such as iOS or Android phones or tablets, some software applications are proposed for contactless fingerprint acquisition. These systems use the built-in camera of the smartphone to photograph single or multiple fingers using the built-in LED, either flash or continuous, for illumination of the fingers. Some monitor the sharpness of continuously sampled images, capturing each finger separately as the autofocus brings each of the individual fingers into focus. Others use a fixed focus triggering capture when continuous monitoring of image sharpness senses the point of optimal focus. Several devices rely upon the user to tap the screen to manually trigger the capture.

\subsection{Post-Processing of Raw Capture}

Virtually all the contactless capture methods are photographic at their core. Whatever else is done in producing the final output fingerprint, the initial capture is a digital photograph or video capture. This grayscale ${ }^{4}$ image of the finger is not immediately comparable to a conventional fingerprint that is typically digitized from inked impressions on paper cards or captured directly by contact with an FTIR device. Some algorithm or suite of algorithms is applied to convert the photographic representation of the friction ridge structure into the more familiar grayscale representation of dark-toned ridges and lighter-toned furrows (or valleys). Such representations may be binary, i.e. black and white, but both PIV [NILL] and Appendix-F [EBTS] Image Quality Specifications (IQS) require some distribution of gray levels.

This transformation of photographic representations into the familiar grayscale ridge/furrow fingerprint representation is a significant challenge for contactless fingerprint acquisition, and potentially a major source of error.

${ }^{4}$ While some devices capture the finger image in color, it is converted to grayscale prior to subsequent rendering into the fingerprint image. 


\section{Consideration of PIV Basic Requirements}

\subsection{Background}

Early in our efforts at NIST, we decided based on some preliminary examination of contactless devices that the most reasonable performance target for contactless would be for PIV application and that applicability for forensic application should be reserved for later. The PIV specification [NILL] applies mainly to single finger personal identity verification in one-to-one comparisons. Most contactless devices capture four fingers simultaneously which could be appropriate for identity verification, but applications most useful to the United States Government would involve at least limited capacity for oneto-many identification, e.g. against a watch list database. Appendix F [EBTS] is the more stringent of the two quality specifications, and certification under that standard would imply that images captured by certified devices should be capable of one-to-many machine matching for identification against large databases and forensic level comparison involving latent fingerprint matching with images of a quality suitable for submittal as courtroom evidence.

While not immediately applicable to examination of contactless (also referred to as touchless) fingerprint acquisition devices, it is useful to consider some of the main attributes covered in the PIV specifications to determine, where possible, image quality analogs to the existing standard.

\subsubsection{Capture Size}

PIV IQS [NILL] calls for a single-finger capture dimension of at least $12.8 \mathrm{~mm}$ wide $\mathrm{x}$ $16.5 \mathrm{~mm}$ high. This metric may not be relevant to contactless devices. A number of contactless devices capture four-fingers (or possibly two - index and middle) and then segment into individual fingerprints surrounded by variable areas of white space. Several devices are equipped to capture thumbs or single fingers via selection of appropriate operational modes as well. Some capture fingers sequentially and then arrange them into a four-finger slap ${ }^{5}$ array for storage in addition to individual prints.

\subsubsection{Sequence Control}

A potential issue for some contactless devices may be that of sequence control. To the extent that the four-finger plain impression ("slap") is retained and includes sufficient area of fingers to identify the hand to which it belongs, sequence checking may be possible. In this regard, it should be advised that the slaps be retained as well as the segmented fingerprints, or that sequence checking be required at some point. We will discuss later other reasons for retaining the original captured photograph as well as the rendered fingerprint images.

\footnotetext{
${ }^{5}$ Fingerprint impressions formed by simultaneous capture of the four fingers on either hand (thumbs excluded) are generally referred to as "slaps". These are "plain" impressions in contrast to "rolled."
} 


\subsubsection{Native Resolution}

True optical resolution of the device must be at least $500 \mathrm{ppi}^{6}$. If the capture resolution is greater than $500 \mathrm{ppi}$, it must be downsampled to 500 ppi using appropriate low-pass filtering prior to resampling. ${ }^{7}$ In no case is it acceptable to capture a fingerprint at a sample rate less than 500 ppi and then up-sample to 500 ppi.

Control of sample rate may be difficult for contactless devices that lack constrained or well-defined capture volumes, such as some devices that use built-in cameras of iOS or Android mobile devices. Such devices typically aim to fill a spatial template with fingers attempting to control capture distance. Such "template-filling" fails to compensate for differences in hand/finger size and imprecision in finger placement at the instant of capture. Some vendors may have responded to the scaling requirements by attempting to assess scale of captured images drawing camera focus data from the device camera controller or fixing the focus at a predetermined distance and automatically capturing the image at the point of maximum sharpness as the device (or hand) is moved nearer and farther apart. The image captured at a known scale may then be resampled to the desired 500 ppi. Applications designed to operate on multiple devices, would have to be adjusted to consider the particular camera attributes. Hence, evaluation for certification would have to be performed on a device-by-device basis as is already the case in such certification. In the case of smartphones, however, this presents an additional challenge given the rapid turn-over of smartphone devices. (In our exploratory effort, we have already encountered the problem of securing devices replaced on the market by new models.)

\subsubsection{PIV Aim-Point Resolution Scale}

The PIV IQS acknowledges that while primary resolution may be 500 ppi, actual resolution may vary as a result of perturbations of imaging, e.g. pixel spacing irregularities, scanner misalignment, etc. The PIV IQS allows variation between 490 ppi and 510 ppi, but never less than 490 ppi. In the case of contactless imaging, the problem may be more pronounced due to imaging of out-of-plane sides of fingers at minimum. As will be seen below, yaw and pitch of the finger within the capture volume can also create undesirable variation in sample rate for a single finger image or that across the multifinger capture.

For devices that simply record a plain impression of the distal phalanges, the texture on the sides of the finger will tend to exhibit compressed ridge spacing and loss of detail as a result of being viewed obliquely. Assuming the native sample rate is 500 ppi, the sample rate of this compressed ridge region should remain within the defined limits.

For devices that attempt to "unwrap" the curvature of the finger, a dilemma is encountered. If the native scan rate is 500 ppi, the sides of the fingerprint, upon being unwrapped, have actually been under-sampled. In order for the sides to be sampled at 500

\footnotetext{
${ }^{6}$ Resolution values for friction ridge imagery are specified in pixels per inch (ppi) throughout this document. This is based on widely used specification guidelines for such imagery and is accepted as common nomenclature within the industry. SI units for these will be presented only once. $500 \mathrm{ppi}$ is approximately $197 \mathrm{ppcm}$.

${ }^{7}$ NIST guidance on downsampling 1000 ppi to 500 ppi may be appropriate NIST SP500-289, SP500-306
} 
ppi upon being unwrapped, the original sample rate would have had to approach $700 \mathrm{ppi}^{8}$. This will be a characteristic for which some variance with PIV IQS will need justification. The unwrapping is an attempt at generating the desired "nail-to-nail" representation. Unwrapping raises a series of important questions. Is it working sufficiently well to justify the effort? - or to give credit to the attempt? Is useful information provided in the region at the lower resolution?

\subsubsection{Gray Level Output}

PIV IQS specifies that all test target and fingerprint scans be output as $8 \mathrm{bpp}, 256$ gray level monochrome images. It further specifies that operational fingerprint polarity is expected to be dark gray ridges with light gray furrows.

This latter requirement may become among the most difficult and controversial of the requirements. Most contactless devices encountered thus far exhibit some degree of polarity reversal or ambiguity over the fingerprint surface. Whereas contact devices leave little doubt over the locations of highest local relief, as these are points of contact, relief in contactless acquisition is defined by modeling due to lighting. Topographic highs can be either light or dark. Bright areas can be flanks of ridges, tops of ridges, or bottoms of furrows depending upon locally distributed lighting. Were this effect consistent over the entire friction ridge surface, the ambiguity might be easily resolved via post processing. However, this effect changes over the friction ridge surface, often observed to convert a ridge bifurcation into a ridge ending, with small displacement of position if the flanks of ridges are illuminated rather than the ridge crest. Modern matchers are robust to small displacements in minutiae position. We suspect that many may be robust to reversals of ridge type and angle, thus explaining their ability to deal at all with contactless fingerprints. Examples and discussion of this challenge are presented in Section 6.

In most cases, contactless systems collect a raw image and then create at least one or several intermediate images prior to a final output fingerprint representation. In addition to segmentation, additional processing is applied to convert the photographic image into a representation more closely resembling a livescan or inked cardscan capture. The transition from photograph to this ridge/valley grayscale fingerprint is of particular interest in developing the metrology for this new capture modality, as the accuracy of ridge placement and minutiae location may depend on how well the system under evaluation handles the polarity ambiguity problem.

\subsubsection{Noise}

The PIV IQS includes tests of sensor noise, examining pixel value variation observed in otherwise homogeneous dark and light test targets. Block-wise standard deviation of pixel values is calculated over the target area comparing standard deviation values to the threshold value, 3.5 .

For contactless devices such a measure is difficult to impossible to perform with sensors in operational mode. Recall that we are not so interested in testing the camera integrated

${ }^{8}$ We use a triangular approximation here, so to get $500 \mathrm{ppi}$ on the hypotenuse of the triangle (side of finger) we need $500 \sqrt{2}=707$ ppi on the base (plain impression). 
into the contactless system. Rather we are interested in the final rendered output of the device that will be compared to legacy contact exemplars. Accordingly, testing noise of the sensor would bear little relevance to the processed output fingerprint.

However, we have two methods by which to address "noise." The blind signal to noise ratio (BSNR) metric provides a measure of edge strength and the presence of high frequency "speckle" in the measured image. A second look at "noise" takes the form of artificial texture rendered from the smooth (polished) regions of several of the target artifacts. (see Section 7) What we see in some images of several targets is polished regions of the artifacts filled with dots or line segments, which we refer to as "feature noise", introduced by the rendering algorithm as exhibited in Figure 1.

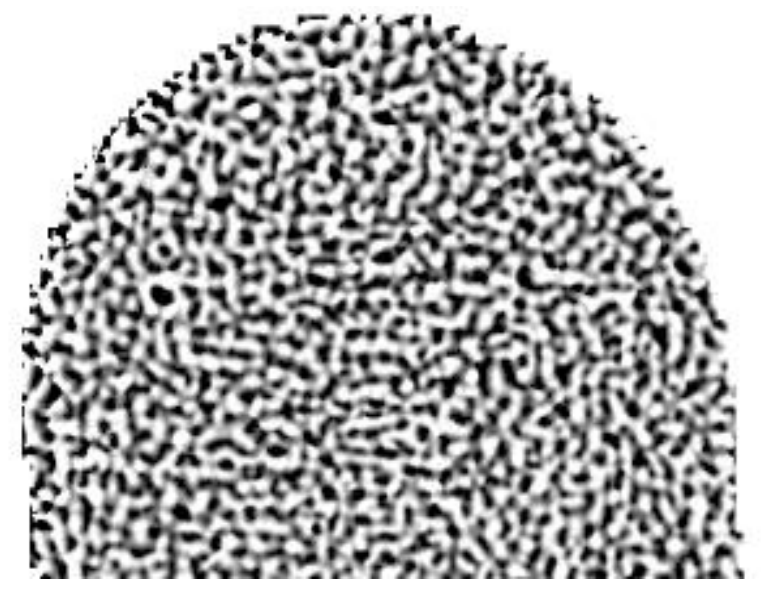

Figure 1 - Image of a portion of one of the target artifacts. Most of the surface of this artifact is polished smooth, yet the rendering algorithm introduces false texture or "feature noise".

\subsection{Geometric Accuracy}

\subsubsection{Ronchi Target Bars}

The PIV IQS specifies several measurements that employ a parallel bar target consisting of bars patterned at one cycle/mm (see Figure 2). The targets are imaged with bars oriented both vertically or horizontally and measurements range from examination of bar spacing to modulation transfer function. Originally used for testing card scanners for the Appendix-F IQS [EBTS], the flat targets now are used for livescan devices. (A "cycle", with respect to the Ronchi target, consists of the width of a black bar plus that of its adjacent white bar.) 


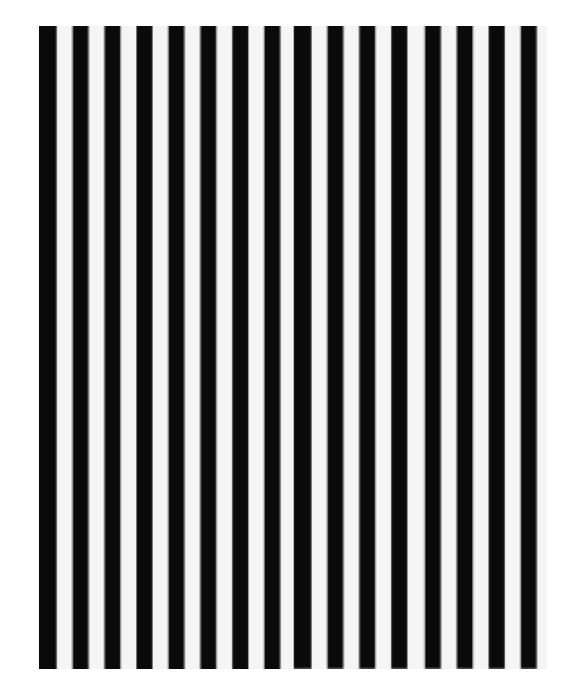

Figure 2-1.0 cy/mm Ronchi Ruling Target (enlarged example of $15.5 \times 19 \mathrm{~mm}$ original)

Regardless of PIV testing on other devices, the flat targets are not appropriate for testing many contactless devices, particularly if capturing images of a target requires disabling key elements of the fingerprint capture mechanism. Most consumer grade cameras these days are more than adequate to capture the Ronchi target at sufficient fidelity to satisfy the PIV IQS. However, the contactless device is more than simply a camera. It is a system with an intended mode of operation. A true test, accordingly, should examine performance as the device is intended to be employed and not as individual components might perform.

Given that most contactless devices capture a photograph, it could be argued that they might be able to capture images of any of the flat targets specified in the PIV IQS. The cameras of such devices may yield accurate renderings of these flat targets sufficient to meet the PIV-IQS standards. However, such accuracy may not persist with capture from a 3D surface subjected to the vagaries of the distribution of illumination. With contactless capture, we have a 3D micro-surface, the textured friction ridge pattern, wrapped onto a curved macro-surface, the finger. Were the contactless capture to end at the photograph for human interpretation, the 3D problem might be lessened. Trained examiners should be able to interpret local illumination variation. However, the post processing that converts the photographic representation to a simulated livescan fingerprint introduces additional sources of error that are not present in conventional contact-based acquisition.

While several of the PIV specifications for the imaging of Ronchi targets are meaningless for contactless sensing, general assessment of bar spacing of both vertically and horizontally constructed bars would be appropriate, given that a 3D Ronchi target is employed. Notions of optical distortions, such as "pincushion" or "barrel", become moot considering that such sensor distortion would be overwhelmed by mere out-of-plane pitch and yaw of the finger during capture. These positioning effects might be assessed using various 3D target artifacts. Such artifacts and applications are described in Appendix CFabrication of Test Artifacts and Section 7. 


\subsection{Spatial Frequency Response}

Contactless images are unlikely to have modulation transfer function and contrast transfer function measurements as specified by the PIV IQS using flat targets, and it is not clear that such measures would be useful without adaptation to targets appropriate for 3D imaging. However, frequency analysis could be applied as part of structural comparison with contact acquisitions of fingers or artificial targets both globally and locally. Variations in frequency response over the image area can reveal inconsistencies in sample rate, contrast, and sharpness.

\subsection{Gray Level Uniformity}

Given there is no suitable 3D equivalent to the targets described in the PIV IQS, detailed evaluation of gray level uniformity on any level becomes difficult to assess for contactless fingerprint acquisitions. Even were one to construct a 3D curved smooth surface artifact to be imaged, one would expect considerable variation in gray level due to irregularities in illuminance of the surface. Indeed, almost any lighting configuration of such a surface, other than that within an illumination sphere, would be expected to display regions of specular reflection as well as those darkened by reflection of incident light away from the sensor or directionality of the light source.

\subsection{Fingerprint Image Quality}

\subsubsection{Fingerprint Gray Range}

The PIV-IQS specifies that at least $80 \%$ of twenty captured fingerprint images shall have a grayscale dynamic range of at least 150 gray levels. In practice, this translates to a histogram having at least 150 of 256 bins containing at least five pixels. Such a measure could be applied to contactless captures or an alternative such as image entropy applied with some adjustment, as will be explained later.

\subsubsection{Fingerprint Abnormalities}

Abnormalities in contactless fingerprints might be difficult to evaluate unless the final, rendered prints are somehow compared to photographic images of the fingers. An alternative might be to compare the contactless renderings to impressions acquired using a certified livescan contact device.

\subsubsection{Fingerprint Sharpness and Detail Rendition}

The PIV-IQS specifies only that sharpness and detail rendition should be sufficient "to support the intended applications." In the case of the present study we address this with other attributes in the context of interoperability with contact captures. 


\section{Image Quality and Interoperability Metrics}

\subsection{General Approach}

\subsubsection{Subjects and Fingerprints}

Due to the exploratory nature of the study, our aim was to collect contactless fingerprints from relatively few subjects to provide a small suite of test images by which to develop an analysis methodology. Accordingly, initial data were obtained during several pilot studies conducted by NIST investigators. Approximately sixty NIST personnel volunteered to participate in a contactless device usability study, described in [NIST3]. Each of these subjects provided four fingerprints from the right hand for three contactless devices and two encounters of slap-four impressions on an FTIR device for comparison.

A second pilot study was sponsored by the Intelligence Advanced Research Projects Activity (IARPA) to assess throughput for a planned study that will involve acquisition of fingerprints on a large number of devices. This pilot study provided two encounters of left and right-hand slap-four impressions acquired with a smartphone application and corresponding FTIR fingerprint images for each participant [NIST6].

To establish a substantial interoperability comparison baseline, we drew from image data provided to us by West Virginia University that was collected from multiple contact devices used in the 2014 NIJ Phase 2 study [NIJ1] of contactless fingerprint acquisition. Approximately, 300 subjects were involved in this NIJ study. The slap-four images were segmented, and we selected 1200 fingerprint images from this study for each of three devices and of one set of scanned, inked samples from 10-print cards.

\subsection{Fingerprint Acquisition Devices}

\subsubsection{Contactless Devices}

Toward preserving the privacy of device manufactures entering Cooperative Research and Development Agreements (CRADAs) with NIST, we identify the three touchless ${ }^{9}$ devices ${ }^{10}$ used on our investigation only as TA, TB, TBrp, and TM, representing two stationary, or portal-type, devices (TA and TB) and one Android smartphone application (TM). The device designation TBrp refers to raw photographic images of TB reprocessed using an alternate rendering algorithm.

\subsubsection{Contact Devices}

Two FTIR devices were employed to acquire control contact fingerprints from subjects providing contactless samples of the usability study and the MD Test Facility pilot. One

\footnotetext{
9 We use the terms "touchless" and "contactless" interchangeably.

10 The contactless devices used to collect data appearing in this report are under development. The analyses presented herein reflect the state of these devices at the time the data were collected and are not reflective of current or potential future performance. The analysis of the small samples of fingerprint images are intended only to serve as the medium by which to examine and demonstrate the behavior of candidate measurements and analysis procedures being developed by this NIST project.
} 
of these devices, $\mathbf{C 1}$, was used to acquire a second slap-four impression from the approximately 60 NIST volunteers. A second FTIR device, C2, was used to collect slapfour impressions of both right and left hands to correspond to those collected with the Android smartphone application designated TM.

Three contact devices selected from the NIJ Phase 2 study included two FTIR devices, C3 and C4, and a mobile contact device designated C5. C6 refers to scanned, inked 10print cards. Table 2 provides a summary of the devices and the technology employed by each.

All fingerprint impressions were either provided by devices individually or resulted from segmentation of four-finger slap impressions. For the current study, only plain impressions were evaluated. While true that some contactless devices capture greater friction ridge area than the plain impressions segmented from the slap-four captures, this initial examination of contactless devices considered all in the context of ID flats ${ }^{11}$. Only plain impressions of fingers were considered as the contactless devices examined are not ideally suited for capture of thumbs ${ }^{12}$.

Table 2 - Summary of Devices and Application In Study

\begin{tabular}{|l|l|}
\hline \multicolumn{1}{|c|}{ Device } & \multicolumn{1}{c|}{ Technology } \\
\hline TA & Touchless - Optical + Structured Light \\
\hline TB & Touchless - Photographic \\
\hline TBrp & Touchless (B)- Photo Alt. post-processing \\
\hline TM & Touchless - Photo Android smartphone app \\
\hline C1 (encounter 1) & Contact - Optical FTIR \\
\hline C1a (encounter 2 ) & Contact - Optical FTIR \\
\hline C2 & Contact - Optical FTIR \\
\hline C3 & Contact - Optical FTIR \\
\hline C4 & Contact - Optical FTIR \\
\hline C5 & Contact - Mobile - Electroluminescent \\
\hline C6 & Contact - Scanned ink on paper \\
\hline
\end{tabular}

\subsubsection{Fingerprint Images}

As mentioned previously, the analysis to follow applies to fingerprint images collected as part of several NIST studies. One such study examined usability of three stationary contactless capture devices [NIST3]. The usability study was aimed at determining the extent to which 60 naïve users of the devices could each effect capture of a set of four fingerprints of their right hands under varying degrees of instruction or supervision. A "slap-four" impression was acquired from a legacy contact FTIR device (C1) for

11 "ID flats" (or "identification flats" refers to the collection of plain impressions of four fingers of a hand and of the thumb.

12 The contactless devices examined all capture four fingers simultaneously. Several provide for thumb capture as a separate acquisition, but the operation is rather cumbersome. To maintain consistency across devices, we omit thumbs from our analysis. 
comparison. A second impression collected from the device $\mathbf{C 1}$ is designated C1a. The C1 to C1a comparison served as the experimental control for the study.

A second collection exercise provided four-finger images from each of right and left hands using the smartphone application (TM) with comparison prints acquired using $\mathbf{C 2}$. The Mobile device images were acquired with the subjects presenting their hands at two different positions, outstretched horizontally and held upright, thus yielding two encounters. For the present study, the prints from these two collections were combined without differentiation in the analysis.

The usability study [NIST3] provided contact control comparisons using two encounters of each finger acquired on the identical device We wished to develop a more realistic baseline to represent contact-to-contact interoperability. Accordingly, we selected images from the NIJ Phase 2 study [NIJ1] that were acquired using four different legacy contact devices. Impressions acquired from one of these, C3, were designated the exemplars, and corresponding fingerprint impressions from $\mathbf{C 4}, \mathbf{C 5}$, and $\mathbf{C 6}$ served as queries (probes) for baseline contact-to-contact comparisons. Examination of Table 3 shows the number of samples collected from each of these contact devices. The $1000+$ contact-to-contact comparisons for these devices provides a respectable assessment of interoperability among legacy fingerprints capture methods.

Interpretation of the analysis provided by measurements in later sections, must be conditioned with respect to the disparate sample sizes of the fingerprint samples. Only the NIJ Phase 2 samples of contactless included a substantial number of fingerprint images (over 1 000). Fingerprint sample counts from devices is shown in Table 3.

Table 3 - Fingerprint samples from eac
\begin{tabular}{|l|r|}
\hline \multicolumn{1}{|c}{ Device } & Samples \\
\hline TA & 184 \\
\hline TB & 176 \\
\hline TBrp & 86 \\
\hline TM (encounter 1) & 244 \\
\hline TM (encounter 2) & 223 \\
\hline C1 (encounter 1) & 232 \\
\hline C1a (encounter 2) & 232 \\
\hline C2 & 244 \\
\hline C3 & 1199 \\
\hline C4 & 1199 \\
\hline C5 & 1129 \\
\hline C6 & 1078 \\
\hline
\end{tabular}

13 Note that to increase sample size, multiple encounter samples are combined in the analysis except for C1 and C1a, the experimental control. 


\subsection{Image Registration}

To facilitate a variety of comparisons between contactless acquisitions and those using the contact device, corresponding pairs of images are spatially aligned or registered with one another. We anticipate eventually the use of a fully automated method that combines the automated registration with analysis, but for the purposes of the present exploratory work, we employ a method that determines the affine transformation that will bring the two images into registration based on control points, selected using a proprietary feature extractor.

As will be seen in Section 5, we register images for some of the metrics to ensure that comparison is made between similar regions of the ridge structure, i.e. where the pattern details are as closely matched as practical, and that the metrics are applied to approximately equal-sized samples of the fingerprints under comparison.

\subsection{Control Point Selection and Transform Refinement}

We use a proprietary fingerprint matcher that provides, in addition to a fingerprint match score, a list of corresponding minutiae that provide candidate control points for automated image registration. For each image pair, the proprietary tool outputs multiple sets of corresponding minutiae for the input image pair, hence we refer to it as "the correspondence tool". We designate one image the query image and the other the exemplar. The transform estimation routine of MATLAB [MATLAB] requires two pairs of points that correspond between the images to be registered. The correspondence tool supplies at least two pairs of corresponding minutiae, but in most cases additional choices. Then for each pair of points provided for the exemplar image, our registration scheme (written in MATLAB) cycles through each of the available pairs of points for the query image to generate multiple candidate sets by which to compute a registration transform. Each combination of candidate control points is used to compute a candidate affine transformation. Each computed transform is applied to all combinations of control points a displacement distance is computed between the corresponding pairs of now registered control points. In each case, we compute the average displacement between the registration points to select the candidate transform that yields the minimum average displacement. We apply this affine transform to the register the two images. The average control point displacement for the optimum transformation is recorded as one of our comparison metrics, i.e. the mean minutiae displacement.

\subsubsection{Registration, Minutiae Position Disparity, and Scale Measurement}

The affine transform is a $3 \times 3$ matrix that includes a scaling factor. As we desire only rigid rotation and translation, the scaling factor is removed from the transformation before its application to the image, to be designated as the "moving" image. The scaling factor is recorded as another of our metrics. It serves as an indicator of differences in sample rate between the images under comparison.

To ensure that the transformation does not translate the "moving" image beyond the borders of the "fixed" image, we add a padding of fifty pixels to the borders of the 
"fixed" image. The transformation adjusts the size of the rotated/translated ("moving") image to match that of the "fixed" image. Thus, the result of the process is a pair of images that are identical in dimensions and having the fingerprint content in "best-fit" spatial registration. Figure 3 exhibits a plain (flat) fingerprint ${ }^{14}$ impression in registration with its rolled mate. Both prints are acquired from a contact device.

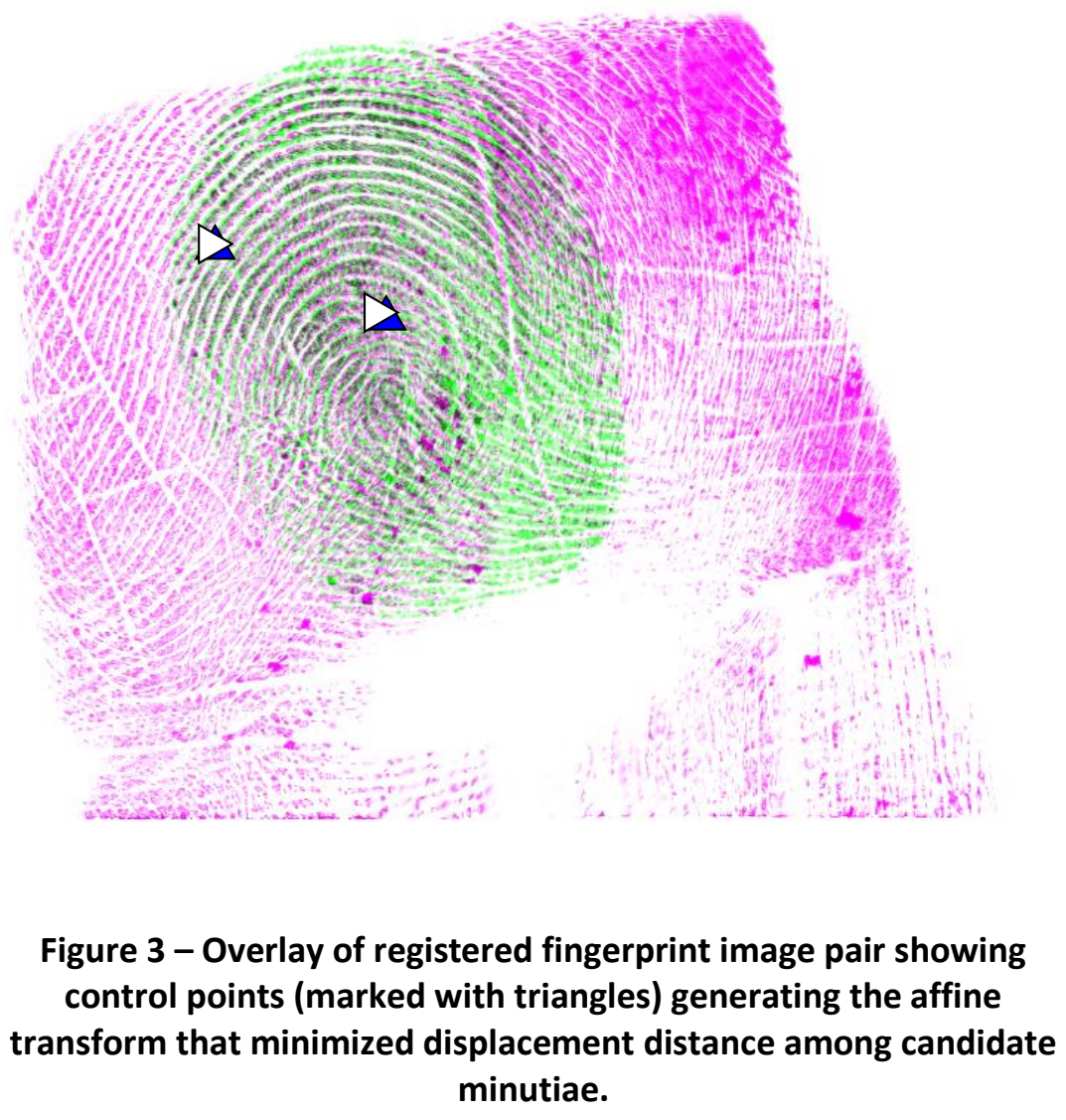

\subsection{Overlap of Fingerprint Areas}

Two very basic observations are made in comparing contactless captures with contact derived fingerprints. First, the contactless capture may extend beyond that of the plain contact impressions used for comparison in the present investigation. Second, the contactless capture provides little constraint on the position of the fingerprint at the time of capture. That is, while a contact slap-four may include fingers that are rotated slightly or tipped toward the finger tips to under-sample the plain impression, the necessity to make contact with (touch) the capture surface limits the possible variability in both area and position on the finger of the acquired sample.

\footnotetext{
${ }^{14}$ Fingerprints depicted in this document were collected under an approved NIST Human Subjects Protection Office (HSPO) protocol, with permission to publish granted by donor.
} 
Contactless devices provide much less constraint on control of the capture region. Thus, we include a measure of the overlap between contactless and contact captures. Moreover, for most comparison metrics considered here, we are interested mainly in the areas sampled in common from the friction ridge surface. Thus, we need to isolate this region in each fingerprint impression under comparison.

We define a procedure by which we can determine the relative areas of each friction ridge surface sampled by the various devices as well as isolating the fingerprint region sampled in common by two devices. We may designate the two images to be compared as IA and IB. Process each image as follows:

Given an input image, such as that in Figure $4^{15}$.

\section{Input Image}

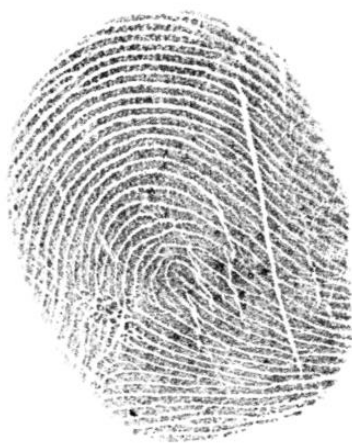

Figure 4- One of two input fingerprint images.

Perform a tophat filter to reduce uneven illumination effects (see Figure 5);

${ }^{15}$ Fingerprints depicted in this document were collected under an approved NIST Human Subjects Protection Office (HSPO) protocol, with permission to publish granted by donor. 


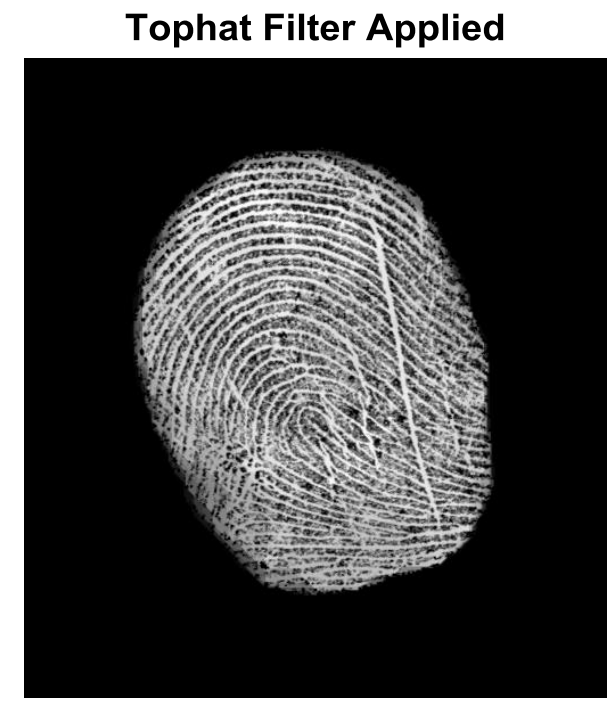

Figure 5 - Tophat filter applied to input image.

Threshold the image to yield binary results, $\mathrm{B}_{\mathrm{A}}$ and $\mathrm{B}_{\mathrm{B}}$, such as that depicted in Figure 6 .

Threshold Applied

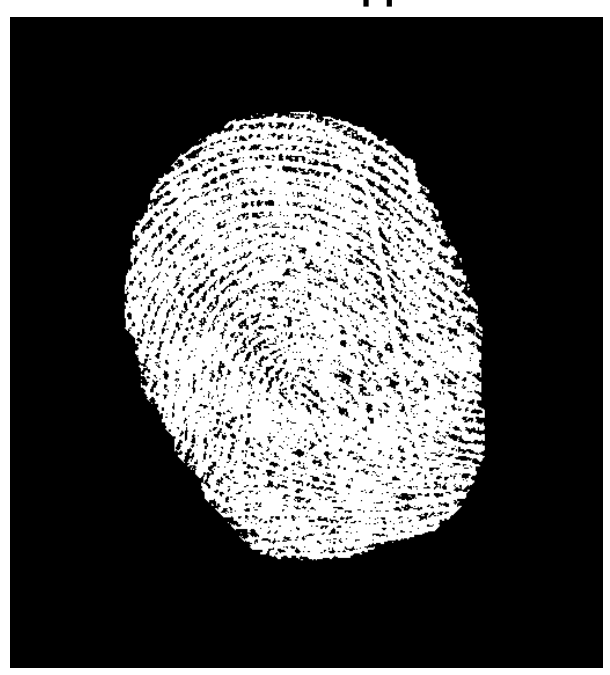

Figure 6 - Threshold applied to binarize the image. 
Apply a morphological "closing" operation to the binary images to remove all textural detail to yield images having the fingerprint background pixels valued zero and the pixels of the fingerprint regions valued one, as shown in Figure 7.

Processed as shown above, the areas of $\mathrm{I}_{\mathrm{A}}$ and $\mathrm{I}_{\mathrm{B}}$. occupied by the fingerprint are then the

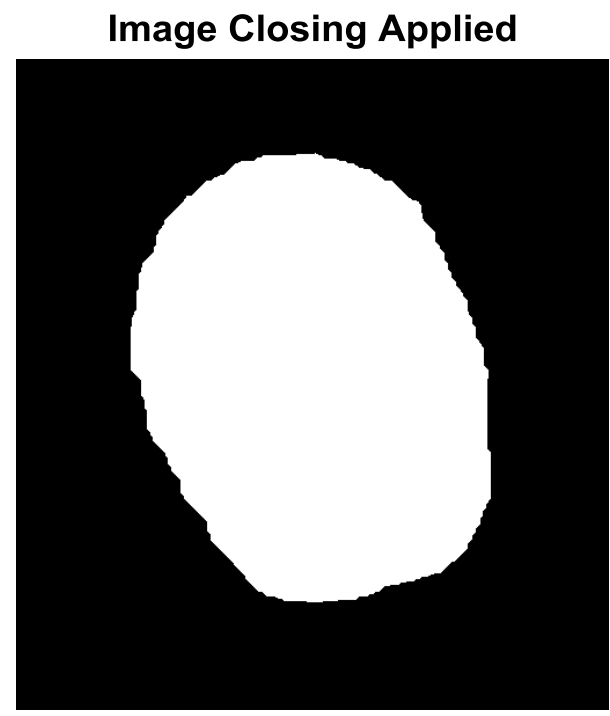

Figure 7 - Morphological "Image closing" operation applied.

sums of the all image pixels in $\mathrm{B}_{\mathrm{A}}$ and $\mathrm{B}_{\mathrm{B}}$.

The sum of $B_{A}$ and $B_{B}$ forms an output image, $I_{C}$, consisting of zero values for the background, ones for pixels of non-overlapping regions, and the value two where the fingerprints overlap as shown in Figure 8. 


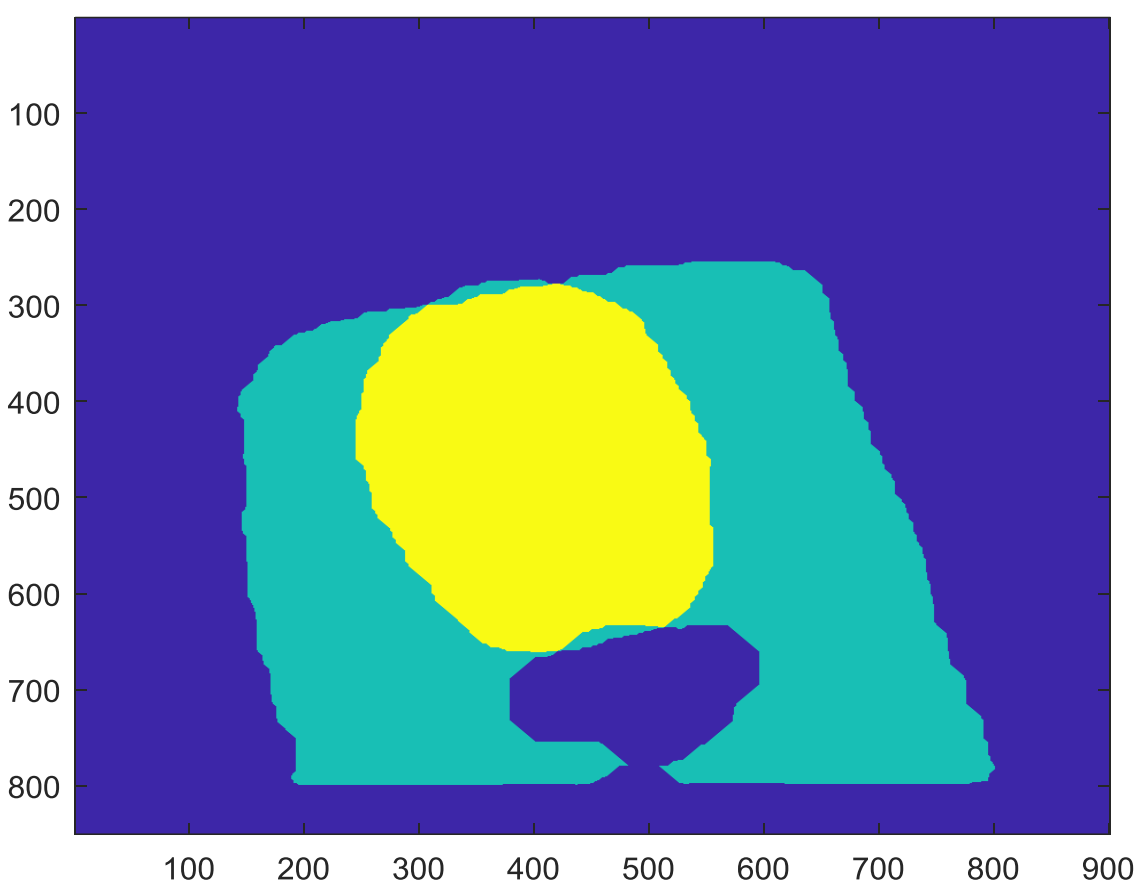

Figure 8 - Having applied the above procedure to the two registered fingerprint images, we sum the binary images yielding an image of values zero where no fingerprint is found (dark blue), one for non-overlapping finger region (turquoise), and the overlapping region summing to two (yellow).

Converting to zeros all pixels in $\mathrm{I}_{\mathrm{C}}$, not equal to two and those equaling two to the value, one, forms another binary image, $\mathrm{B}_{\mathrm{C}}$ representing the region in common or the overlap between the two fingerprints. 
For measures for which we wish to compare only the overlapping regions of the two fingerprints under comparison, delineated as the non-zero pixels in $\mathrm{B}_{\mathrm{C}}$. We use other MATLAB functions to determine the coordinates of the upper left and lower right corners of the smallest rectangle that will enclose the region of non-zero pixels in $\mathrm{B}_{\mathrm{C}}$, such as shown in Figure 9.

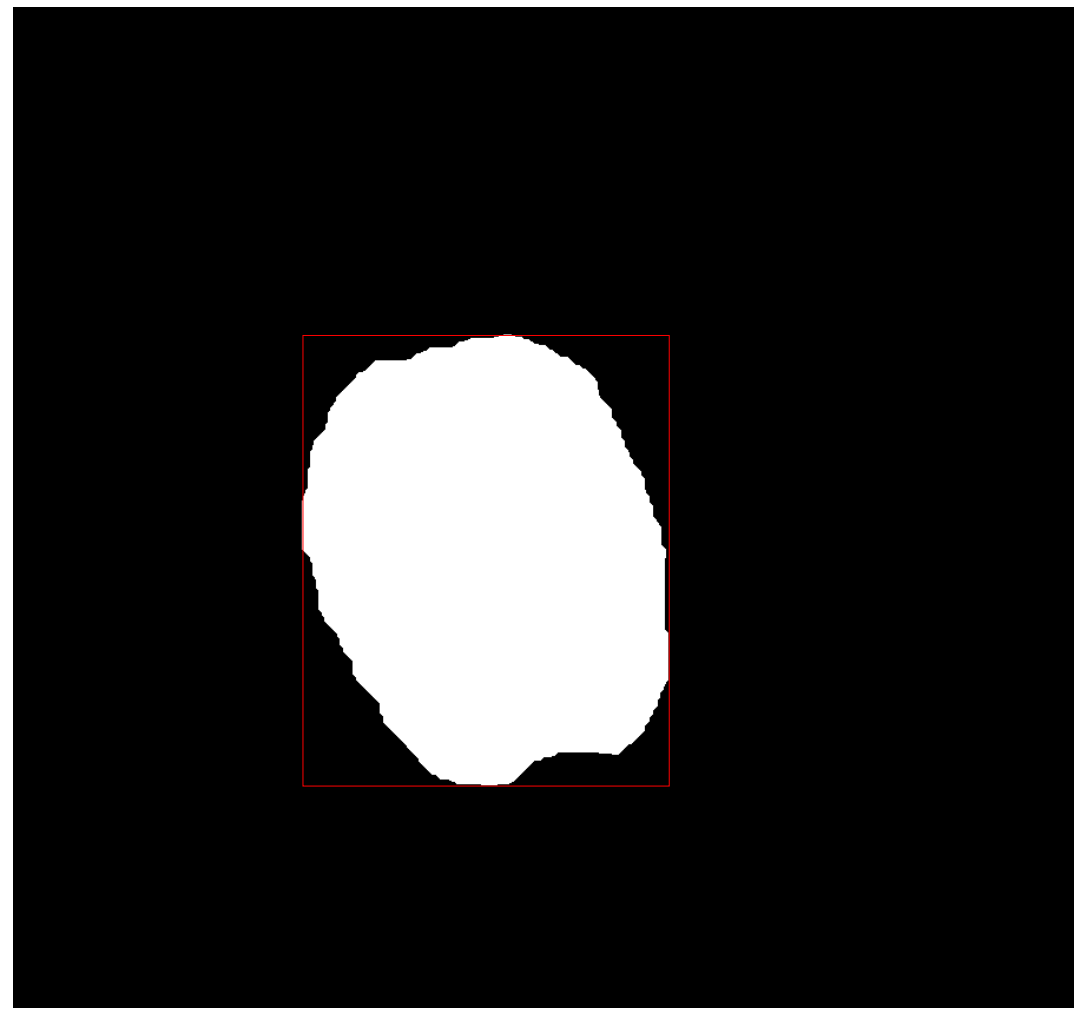

Figure 9 - Locating coordinates designating the smallest rectangle enclosing the non-zero values in $\mathrm{B}_{c}$, gives us cropping coordinates by which to extract the overlapping regions from the original registered image pair. 
Using these coordinates, we can crop the two fingerprint images under comparison to yield $\mathrm{I}_{\mathrm{A}}{ }_{\mathrm{A}}$ and $\mathrm{I}_{\mathrm{B}}$, to which comparison measures are applied. The two cropped fingerprint ${ }^{16}$ samples of the example described above are shown as "img1" and "img2" in Figure 10.
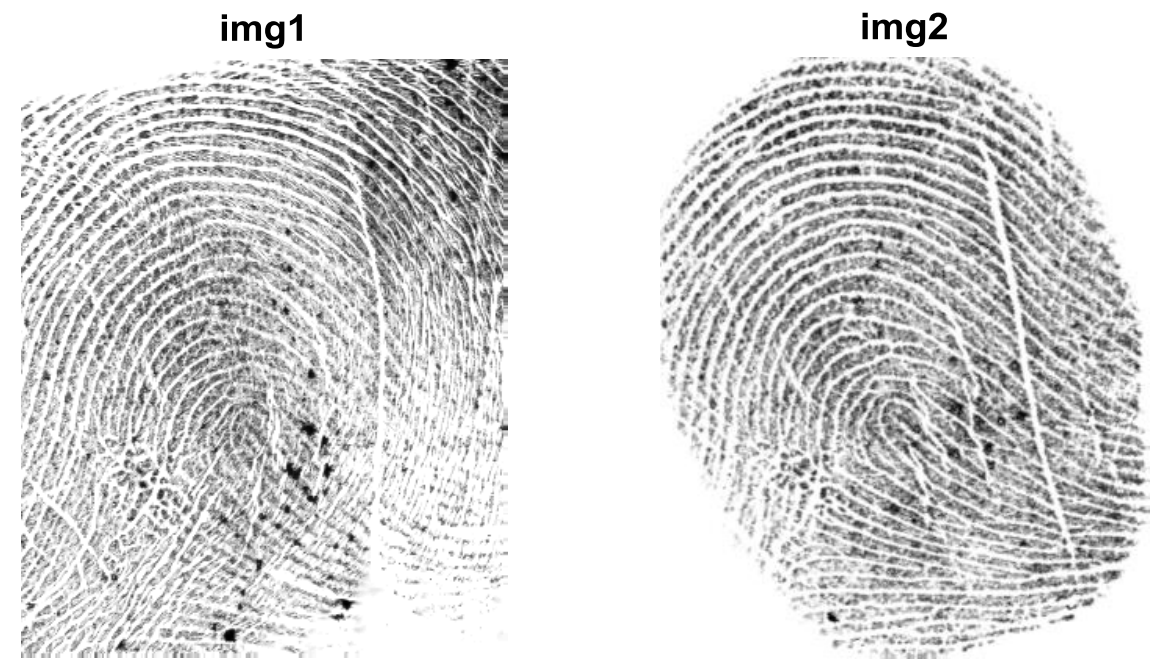

Figure 10 - Overlapping regions of a registered fingerprint pair.

${ }^{16}$ Fingerprints depicted in this document were collected under an approved NIST Human Subjects Protection Office (HSPO) protocol, with permission to publish granted by donor. 


\section{Metrics}

\subsection{The Boxplot (Box and Whisker Plot)}

In the following sections, we describe the various metrics applied to the images and summarize the measurement results in most cases using the data visualization graphic known as the boxplot or box and whisker plot. This method enables simultaneous display of measurement distributions for multiple experimental conditions, or in the present case, devices or comparisons of measurements between devices.
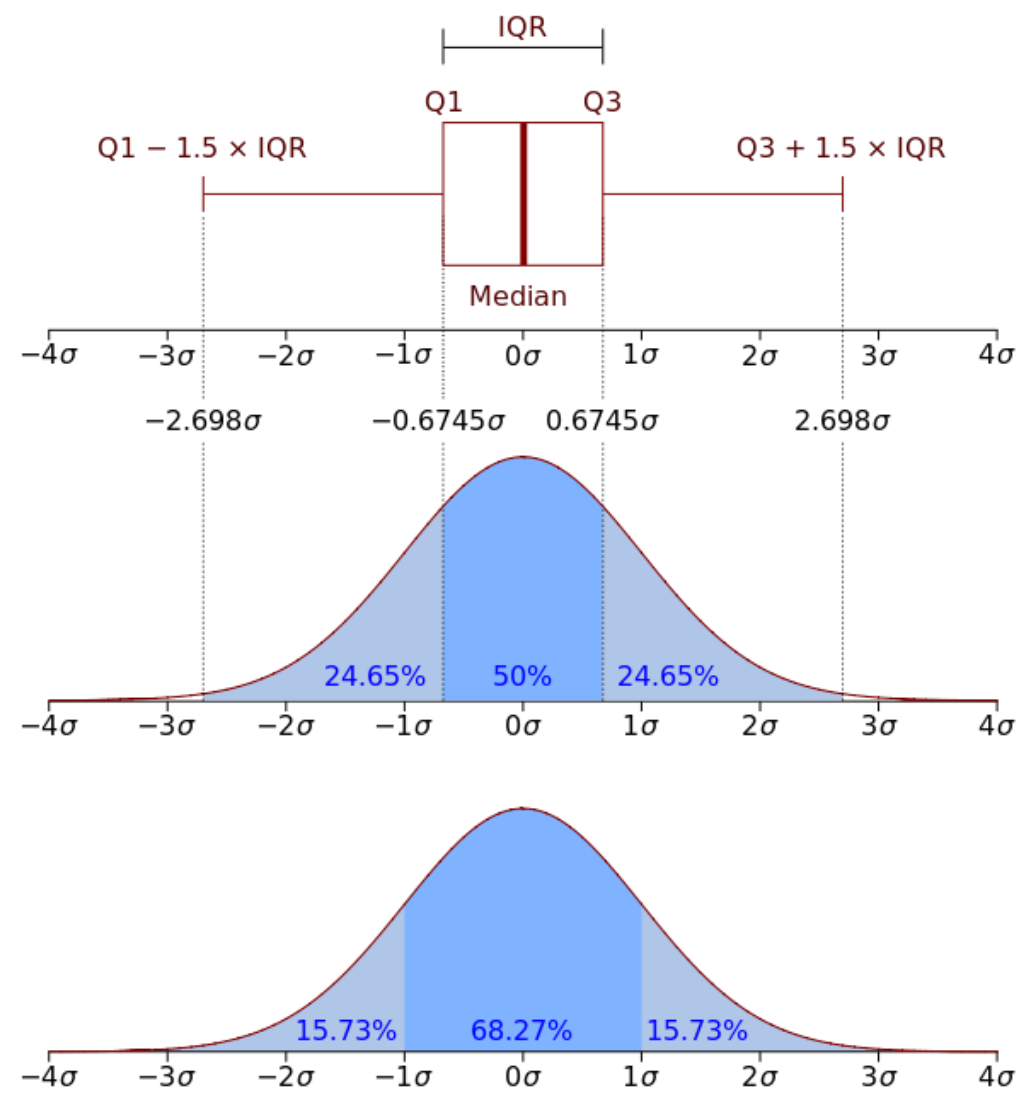

Figure 11 - Relationship between the boxplot of normally distributed data compared to the standard normal distribution for illustrative purposes. (Graphic from [WMC])

Figure 11 illustrates the relation between the boxplot and the more familiar standard normal distribution. We see that the outstanding feature of the boxplot is, of course, the box having the median marked by the central line. In the boxplots used in the present study, the median is surrounded by a notch representing the $95 \%$ confidence interval about the median value. Interpreting the boxplot, it is significant that the box contains $50 \%$ of the distribution and $24.65 \%$ between the limits of the box, Q1 and Q3, and the ends of each whisker. Note that corresponding to the normal distribution, this leaves approximately $0.35 \%$ of the distribution beyond the limits of the whiskers. In the boxplots, these values are outliers and are indicated by red "+" marks. 


\subsection{Single Image Measurements}

We apply a small number of measures to characterize features of both exemplar and query images irrespective of their correspondence ${ }^{17}$.

\subsubsection{Image Entropy}

Entropy $(E)$ provides a measure of the extent to which the image uses the available grayscale. Existing specifications such as [PIV] require pixel values to represent a dynamic range of 150 gray levels. We prefer to describe use of the grayscale using entropy. Intermediate to high entropy tends to be preferred by humans, though it may not be important for machine matching of fingerprint images. We include it here only as a means by which to compare contactless images to those acquired using contact devices.

Entropy is defined as

$$
E_{I}=-\sum_{i=1}^{n} p\left(x_{i}\right) \log _{2} p\left(x_{i}\right)
$$

where $n=$ number of gray levels in the image, i.e. $256 ; x_{i}=$ the value of the $i^{\text {th }}$ gray level; $p\left(x_{i}\right)=$ the probability of occurrence of the $i^{\text {th }}$ gray level in the image. Thus, for a single channel ( 8 bit) image having $p\left(x_{i}\right)=1 / 256$ for every $i$, the maximum entropy is 8 , or 8 bits. Accordingly, an image displaying a relatively flat (uniform) gray level histogram should yield an entropy very near the maximum value of 8 bits. An image having less

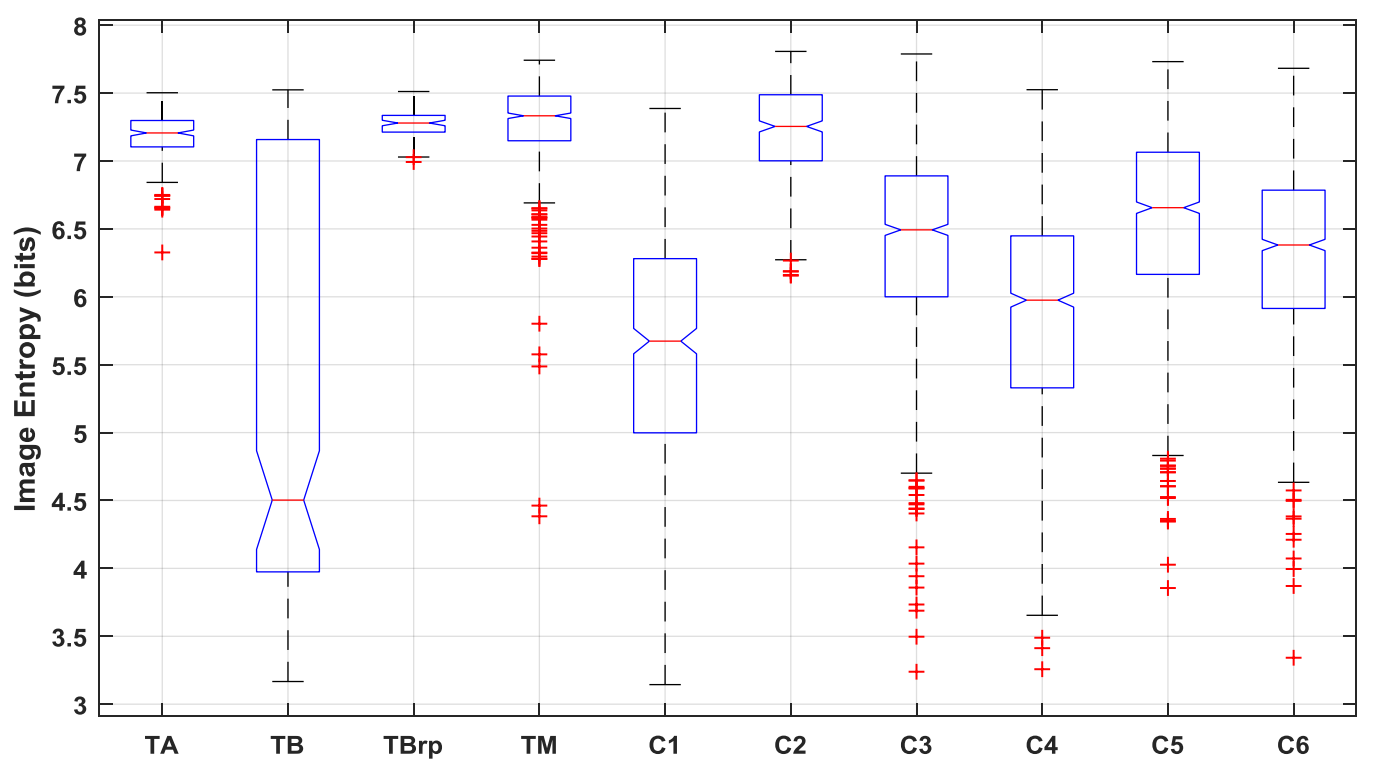

Figure 12 - Image entropy for fingerprint images of each of the devices, both contactless and contact used in the study

\footnotetext{
${ }^{17}$ The contactless devices used to collect data appearing in this report are under development. The analyses presented herein reflect the state of these devices at the time the data were collected and are not reflective of current or potential future performance. The analysis of the small samples of fingerprint images are intended only to serve as the medium by which to examine and demonstrate the behavior of candidate measurements and analysis procedures being developed by this NIST project.
} 
uniform distribution of gray levels, such as an image displaying large areas homogeneous in gray level would be expected to yield a lower entropy measure. This is seen in the very broad distribution of entropy values for Device TB. This device output either binary or grayscale versions of acquired fingerprints. We began collection using the "grayscale" option but found the binary representations better for selection of registration control points.

Other than the anomalous range for Device TB, we see that contactless images display entropy values comparable to or greater than the contact images sampled.

\subsubsection{Blind Signal-to-Noise Ratio (BSNR)}

Zhang and Blum [ZHANG] describe a method for estimating the signal-to-noise ratio of images subjected to some noise or other degrading process in the absence of an original, unprocessed image for comparison. Boult [BOULT] summarizes experiments applying the metric to images corrupted by noise, JPEG compression, and contrast variation. The method involves analysis of the histogram of the edge intensity image, $\|\nabla\|$, i.e. the L2 norm of the gradient of image, I, at each pixel location. Thus, the procedure begins with the computation

$$
\left\|\nabla I_{i j}\right\|=\sqrt{\left(I_{i j}-I_{i(j+1)}\right)^{2}+\left(I_{i j}-I_{(i+1) j}\right)^{2}}, \quad i=1 \ldots n ; j=1 \ldots m
$$

The metric, Q, based on the distribution (histogram) of image gradient values is taken as the proportion of pixels of $\|\nabla I\|>2 \mu$, i.e.

$$
Q=P(\|\nabla I\|>2 \mu)
$$

where

$$
\mu=\frac{\sum_{i=1}^{m} \sum_{j=1}^{n}\left\|\nabla I_{i, j}\right\|}{n \cdot m}
$$

The metric, QR, blind signal to noise ratio, is then given as

$$
Q R=20 \log _{10} \frac{Q}{e^{-\pi}}
$$

The value, $e^{-\pi}$, is the minimum value for a signal consisting of Gaussian distributed noise and is used in the calculation as a base level for the metric - namely as Q approaches the minimum value for a Gaussian signal, QR approaches zero.

In the present instance, the procedure is applied to each of the cropped regions, $\mathrm{I}_{\mathrm{A}}{ }_{\mathrm{A}}$ and $\mathrm{I}_{\mathrm{B}}{ }_{\mathrm{B}}$, common to both fingerprint images under comparison. In interpreting BSNR, one should keep in mind that it favors sharp edges and high contrast.

Distributions of BSNR for fingerprint images of the three contactless devices and of repeat captures from the C1 FTIR sensor are shown in Figure 13. Inasmuch as this measure is responsive to gradients, TB scores highly as most of the images were binary valued and, thus, high contrast. Even in cases where ridge structure is poorly defined, the edges of the segments remained sharp with this sensor. TA, TB and TM displayed varying degrees of high frequency noise in addition to less crisply delineated 
ridges, hence lower BSNR values. The $\mathbf{C} 1$ controls tended toward crisp edges, but some were low contrast captures reducing the BSNR values. Similar responses are observed for the other contact devices, C2, C3, C4, C5, and C6.

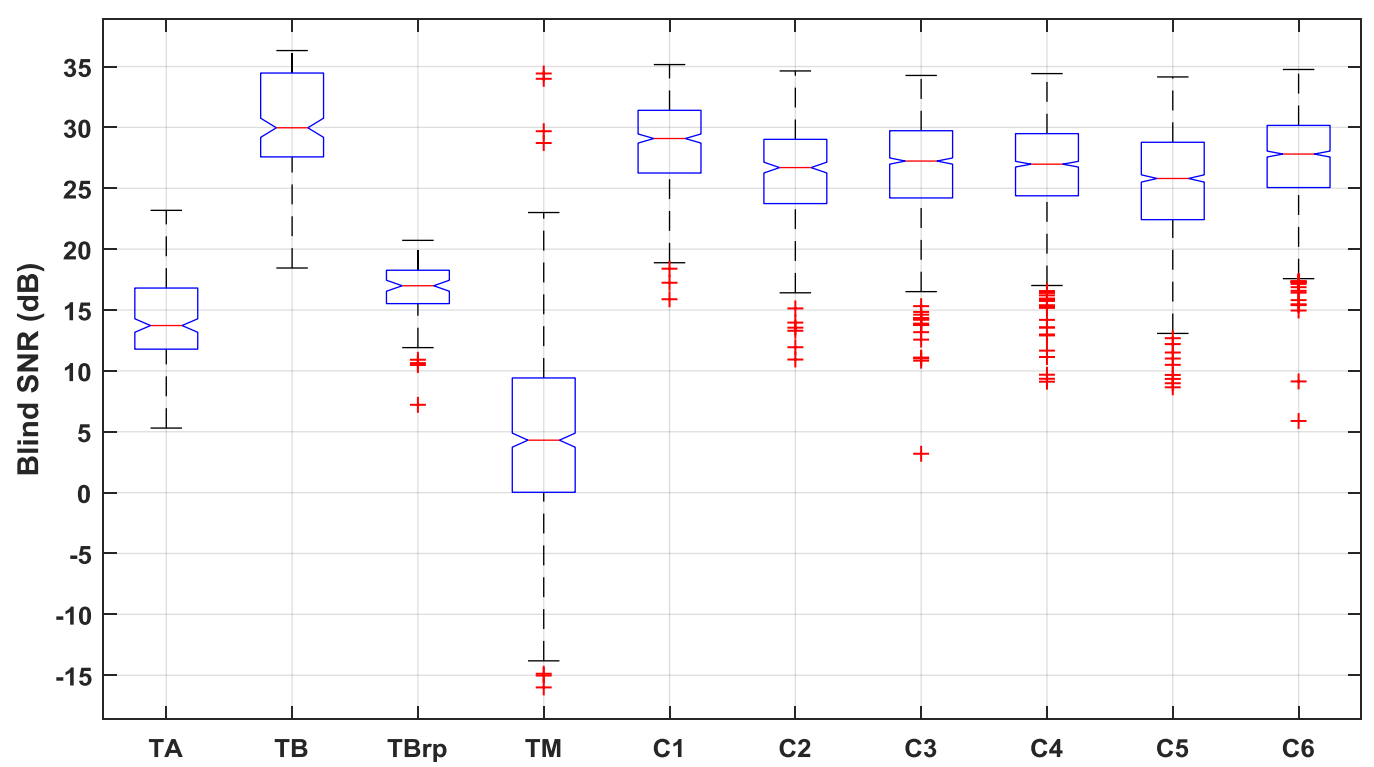

Figure 13 - BSNR values for fingerprint acquisitions from three contactless fingerprint devices and for six contact devices.

\subsubsection{NIST Fingerprint Image Quality (NFIQ) Version 2}

In 2004 NIST developed the first publicly available fingerprint quality assessment tool, NFIQ. Calibrated against fingerprint match performance, the NFIQ metric enabled evaluation of fingerprint samples relative to their relative suitability for recognition. In 2016, NIST, in collaboration with Federal Office for Information Security (BSI) and Federal Criminal Police Office (BKA) in Germany as well as research and development entities, MITRE, Fraunhofer IGD, Hochschule Darmstadt (h_da) and Secunet, issued a revision to the fingerprint image quality standard, NFIQ Version 2.0. NFIQ 2.0 is the basis for a revision of the Technical Report ISO/IEC 29794-4 Biometric sample quality Part 4: NFIQ 2.0 source code serves as the reference implementation of the standard [NFIQ].

NFIQ2 was applied to individual images, contactless captures as well as contact acquisitions. The metric was applied to full-sized, registered images rather than to cropped regions of overlap. Yet for purposes of contactless device evaluation and interoperability, we apply this metric among others described in this document to compare the contactless impressions to legacy contact fingerprints. Figure 14 exhibits distributions of NFIQ2 scores for the three contactless devices and for each set of contact captures. 
As with BSNR we note that device TB appears to perform well with this metric. Once again, this is likely due to sensitivity of the NFIQ 2.0 to edge crispness, pattern clarity,

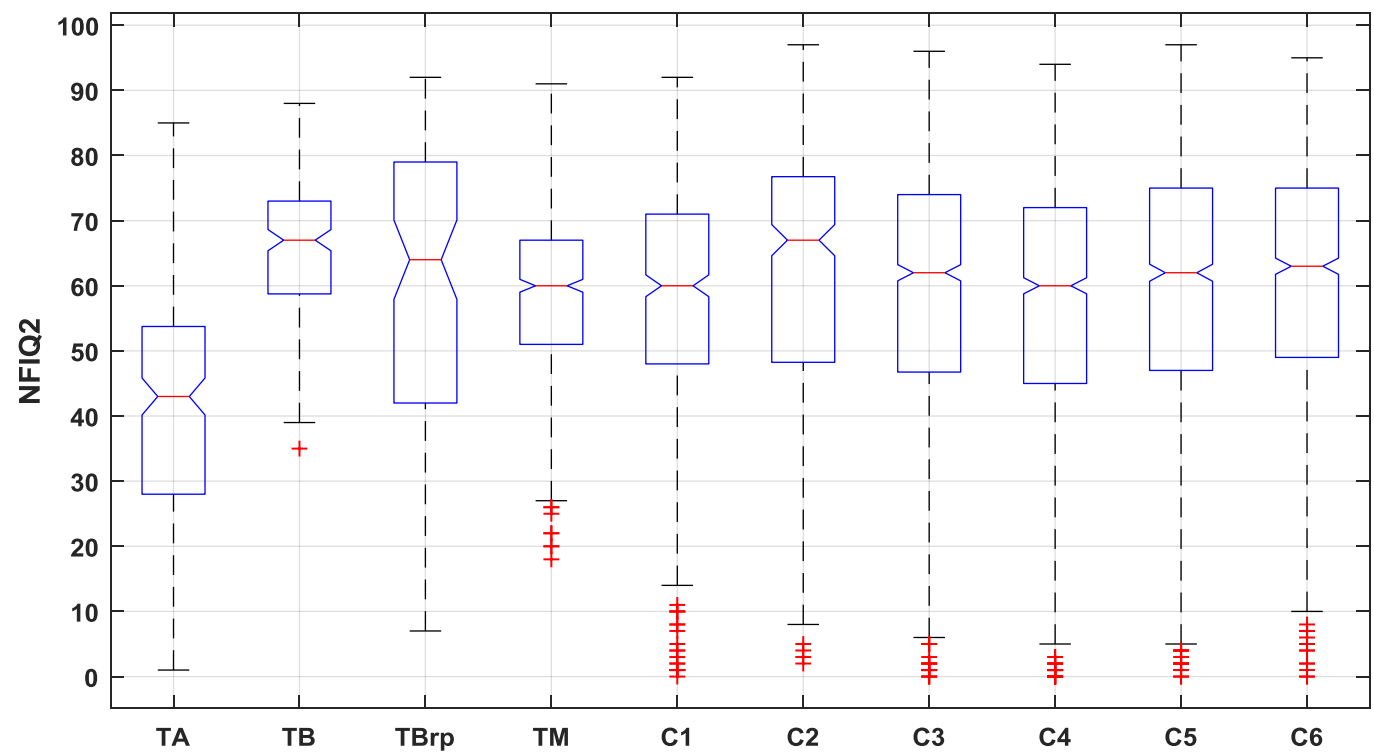

Figure 14 - NFIQ 2.0 scores for acquisitions of each of 3 contactless devices and each of the two acquisitions from the control FTIR contact device.

and feature (minutiae) definition. All other devices follow in median score, followed by device TA showing the lowest median. The contact images show larger variance (larger boxes) but some similarity among them, with extremes above a score of 90 and below 10 . We note that NFIQ2 has not been trained on contactless fingerprint images, nevertheless, it remains a potentially valuable tool for our proposed contactless device assessment.

\subsection{Comparison Metrics}

To help ensure validity of image comparisons with respect to structural metrics, we have registered the images under comparison and cropped out the regions of overlap between the registered mates. All measures below, except match scores, were applied only to the overlapping regions ${ }^{18}$.

\subsubsection{Image Scale/Sample Rate}

The control point registration scheme employed includes scale adjustment as part of its optimization procedure. For our comparisons, we require a rigid transform to include only rotation and translation to bring one image into registration with the other. Of course, we wish to remove the scale adjustment from the transform matrix. The process by which we calculate the scale factor applied during the initial registration yields a useful metric to add to our list.

\footnotetext{
${ }_{18}$ The contactless devices used to collect data appearing in this report are under development. The analyses presented herein reflect the state of these devices at the time the data were collected and are not reflective of current or potential future performance. The analysis of the small samples of fingerprint images are intended only to serve as the medium by which to examine and demonstrate the behavior of candidate measurements and analysis procedures being developed by this NIST project.
} 
We assume our exemplar images as the standard. This may or may not be strictly correct. As will be seen, even contact images can vary in scale relative to those of other devices or even among different encounters from the same device.

As mentioned previously, contactless capture poses some problems with scaling. First, the absence of control over the precise distance of the finger relative to the sensor introduces some variability in scale of the rendered fingerprint. Second, the 3D shape of the finger itself presents the friction ridge pattern at a range of scales. The minutiae selected as control points might be drawn from any part of the rendered fingerprint to be matched to corresponding points of the contact exemplar for which scale is largely constrained by the geometry of the device and its sensor.

In Figure 15 we observe the greatest scale variability with the comparison of the smartphone images to a contact exemplar (TM vs C2). Despite efforts by the application developer to control this, complete success was not achieved. The least scale variability we observe with fingerprint comparisons of two encounters using the identical device, C1a vs C1. Among the contact devices, we see small but increasing variability in scale as we move across technologies. As shown in Figure 15, comparison in order from smallest to largest is FTIR device, C1a vs $\mathbf{C 1}$ and $\mathbf{C 4}$ vs C3, mobile contact electroluminescent device to FTIR, C5 vs C3, and finally inked cardscan to FTIR, C6 vs C3.

Table 4 - Scale variability across contact capture technologies

\begin{tabular}{|l|l|}
\hline \multicolumn{1}{|c|}{ Device type Comparison } & \multicolumn{1}{c|}{ Scale variability } \\
\hline FTIR vs FTIR & low \\
\hline Electroluminescent vs FTIR & Medium \\
\hline Inked cardscan vs FTIR & high \\
\hline
\end{tabular}

We should acknowledge some imprecision in our estimates in that our results reflect relative rather than absolute measurements of image scale and sample rate. While for most image pairs, registration was successful, in other cases some error was noted. We did exclude from the analyses requiring registration cases exhibiting greater than 20 pixels in average minutiae displacement. This 20-pixel threshold was selected liberally to avoid excluding images for which accurate registration might be inhibited by image quality and interoperability issues we hoped to reveal. Thus, we apply the same procedure to both contactless and contactless comparisons and look for relative scale accuracy, noting that registration error may be either cause or effect of scale variation between images under comparison. 


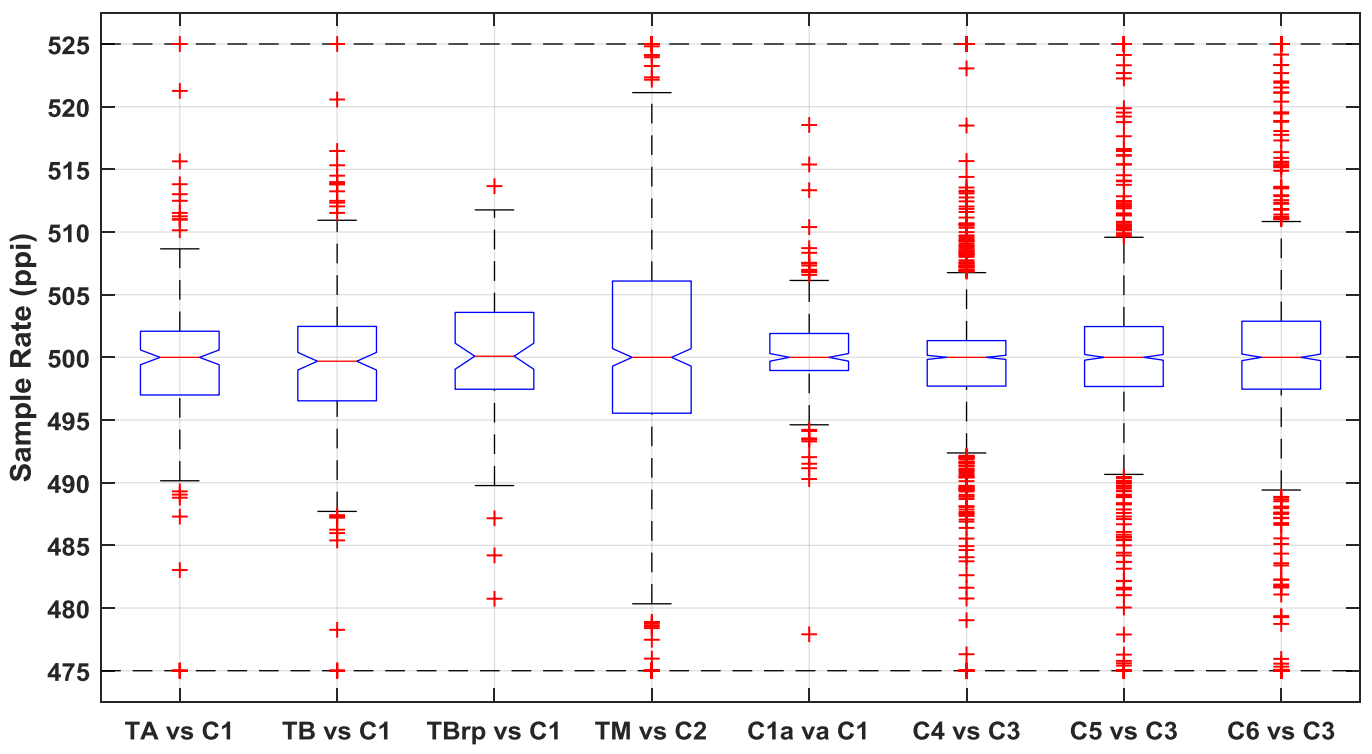

Figure $\mathbf{1 5}$ - We multiply scale factor by 500 ppi to get familiar sample rates for fingerprints. For both PIV and Appendix F, we expect images at 500 ppi. The PIV specification allows values between $510 \mathrm{ppi}$ and $490 \mathrm{ppi}$, and the more stringent Appendix $F$ allows sample rates between 505 ppi and 495 ppi. (Dashed lines indicate exclusion of extreme outliers.)

\subsubsection{Normalized Overlap}

The operations described in Sections 4.3 to 4.5 above provide a measure of the region of overlap between the contactless and contact fingerprint. However, we only here have the area with respect to what? Using the entire image area as a normalizing factor would give us different results for different base image sizes. We would like to see values ranging from zero (for no overlap) to one (for complete overlap of one image with the other.) $\mathrm{We}$ do not expect the fingerprints to be of equal area, so we normalize by dividing the number overlapping pixels by the number of pixels in the smaller of the two fingerprints. This gives us the proportion of overlap between prints having the smallest with that having the largest areas, the value of which will range between 0.0 and 1.0. This measurement we refer to as the Normalized Area of Overlap.

Figure 16 exhibits distributions of normalized overlap between fingerprint image pairs under comparison. Variation in overlap was observed to be largely due to variation in the region of the friction ridge surface sampled by the devices. Such variation for touchless capture results from yaw and pitch of the hand relative to the optic axis of the camera at the instant of capture. Even two contact captures of plain impressions can sample slightly different regions of the friction ridge surface, though for contact devices capturing four fingers together, such variation is limited as all four fingers must contact the recording surface simultaneously. Device TA captures a region larger than that of a typical plain 
impression, hence it covers the region of the $\mathbf{C 1}$ plain impression almost completely in most cases. The lower medians and larger variability for Device TB and TBrp exemplifies one of the vulnerabilities of contactless capture. Namely, the absence of constraint on hand position can yield fingerprints with cores, that would typically be nearly centered in a contact capture, to be displaced sometimes to extremes or to be absent completely from the recorded impression.

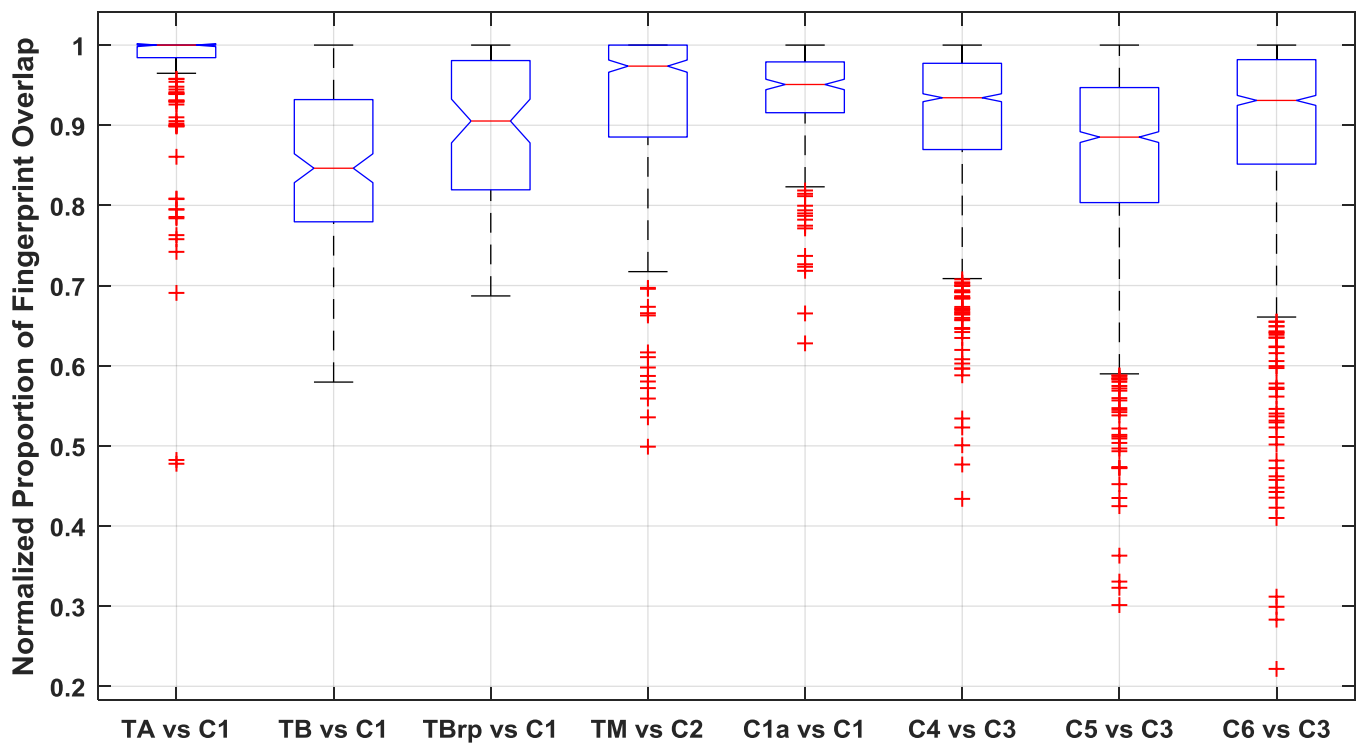

Figure 16 - Overlap between contact fingerprint and corresponding contactless acquisition or between the mated contact acquisitions.

\subsubsection{Frequency Spectrum Comparison}

NIST Spectral Image Validation/Verification (SIVV) was developed initially as a method to screen fingerprint databases for non-fingerprint images, segmentation errors, or mislabeled sample rates. The SIVV metric [NIST1] provides a comparatively straightforward method by which to assess the frequency structure of a fingerprint image. Pairwise display of the SIVV signals of a pair of images enables summary visualization of the effects of differences across the composition frequency spectrum of the image. As a 1-dimensional representation of a 2-dimensional Fourier spectrum, the SIVV metric applied to a fingerprint image exhibits a major peak corresponding to the frequency of the ridge spacing. Also, as shown in Figure 17, comparison of SIVV signals of two synthetic fingerprint impressions shows the difference in spectral power over various frequencies with some low-pass filtering applied to that labeled img1 to yield img2. 
The SIVV signals denoted as s1 and s2 are respectively vectors of SIVV signal values for images under comparison. The frequency samples, $\mathrm{f}$, in units of cycles per pixel correspond to image pixels or Fourier transform frequencies along the length of one half of the minimum dimension of the 2D Fourier transform of the image under examination. Frequency along this dimension is scaled to the interval $[0,0.5]$ cycles/pixel. Note that the power value at $f=0$ is the "direct current" (DC) term, corresponding to the average intensity of the image and is used to normalize the power spectrum.
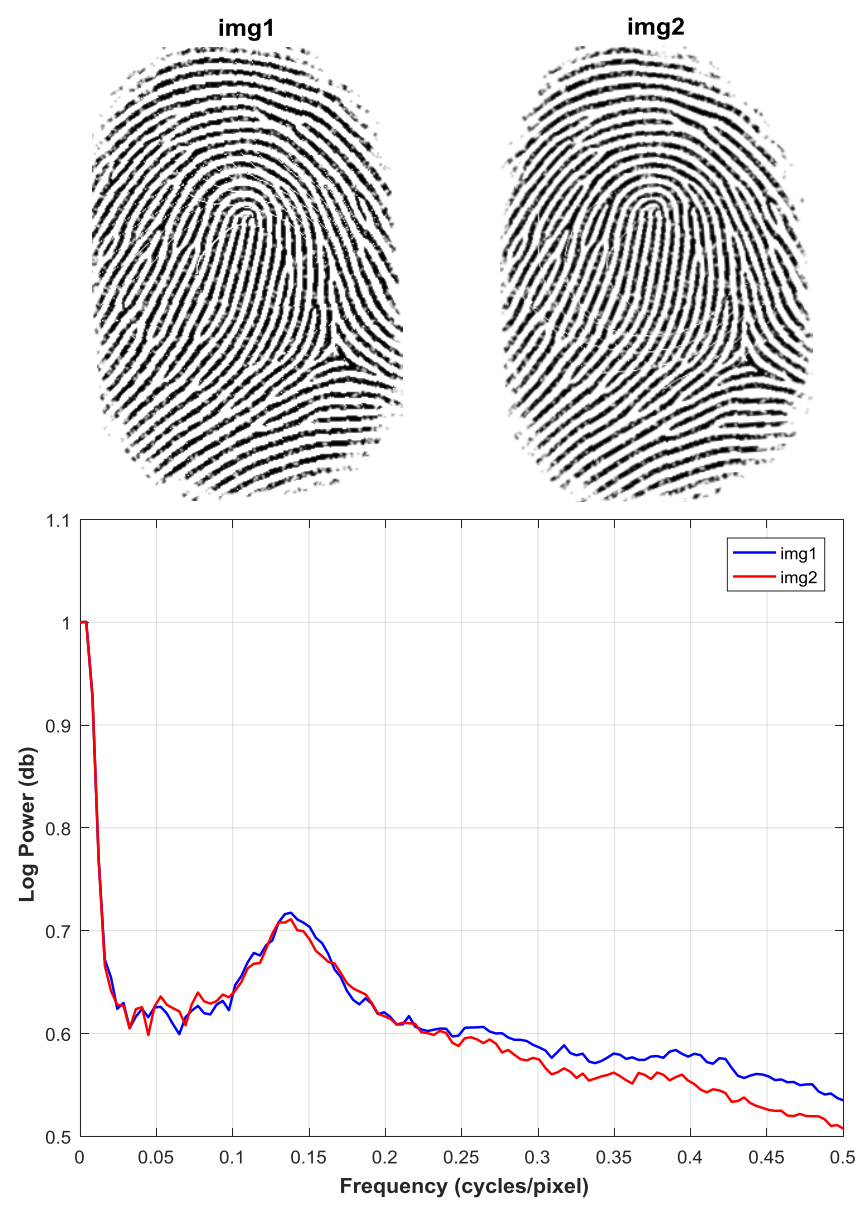

Figure 17 - SIVV spectra of the two (synthetic) fingerprint impressions shown above. Peak location corresponds to spatial frequency of ridge pattern. Applying a small degree of low-pass filtering to img1 reduces power in the high frequencies observed in its processed mate, img2.

\subsubsection{RMS Error of SIVV Signals}

Either differences or ratios of SIVV signals can provide quantitative measures for the comparison of images methods. For the present study, we examine image differences between pairs of images, $\mathrm{I}_{\mathrm{A}}{ }_{\mathrm{A}}$ and $\mathrm{I}_{\mathrm{B}}$, with respect to the Root Mean Squared Difference (RMSD) between their two SIVV signals, s1 and s2, over the entire frequency range 0 0.5 cycles/pixel. 


$$
\operatorname{RMSD}\left(\mathbf{s}_{\mathbf{1}}, \mathbf{s}_{\mathbf{2}}\right)=\sqrt{\frac{\sum_{i=1}^{n}\left(\mathbf{s}_{\mathbf{1}, i}-\mathbf{s}_{\mathbf{2}, i}\right)^{2}}{n}}
$$

where $n=\left|\mathbf{s}_{1}\right|=\left|\mathbf{s}_{2}\right|$ (i.e., the lengths of the signal vectors).

The RMSD metric defined above can provide a measure of the overall difference between the SIVV spectra of images subjected to different processes or, as in the present study, fingerprint impressions acquired using different devices. In addition to global effects, the RMSD may be evaluated over smaller frequency intervals enabling the comparison of effects over frequency bands that may have particular relevance to fingerprint image quality or matching, as well as quantifying and isolating changes confined to bands that specifically impact either the machine matcher or expert examiners.

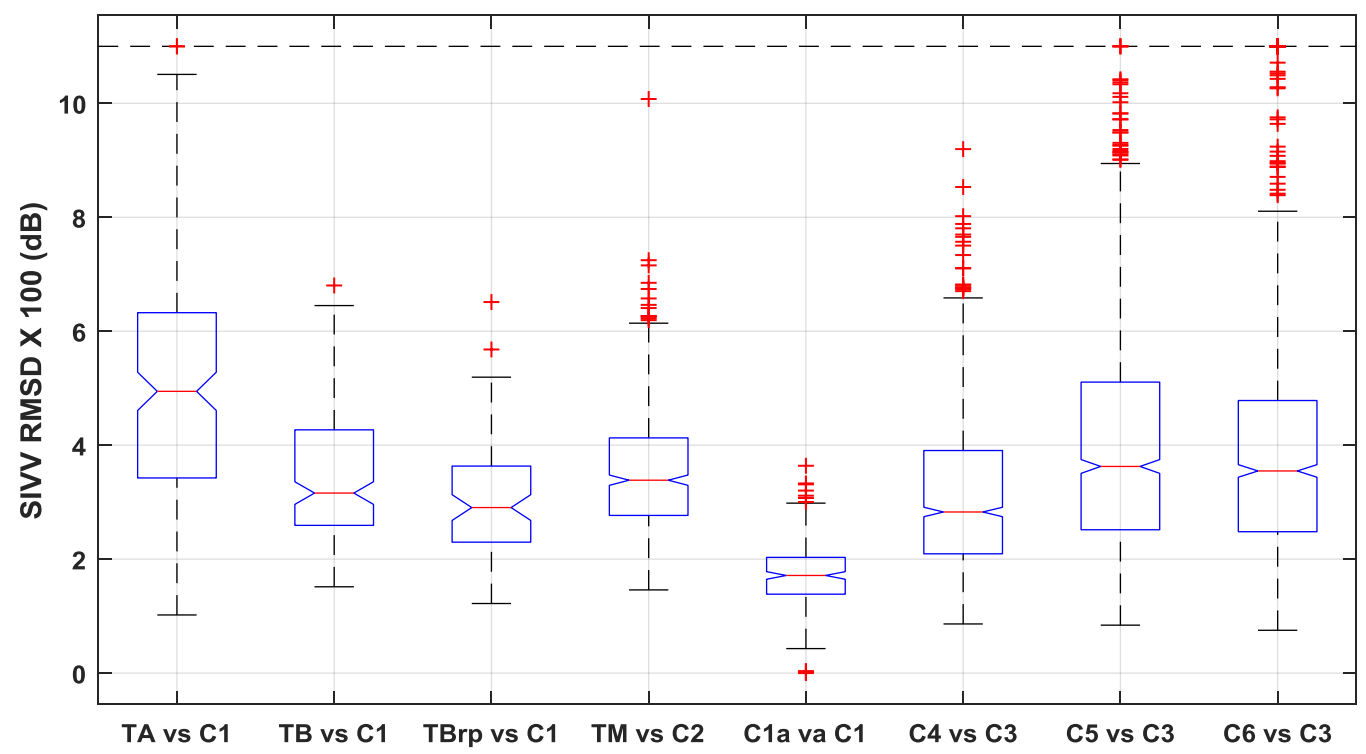

Figure 18 - Root Mean Squared Difference of the spectra of contactless to contact and various contact to contact comparisons. Lower values are better. (Dashed line indicates exclusion of extreme outliers.)

For the present purpose, we compute the RMSD for corresponding cropped images, $\mathrm{I}^{\prime} \mathrm{A}$ and $\mathrm{I}_{\mathrm{B}}$. Figure 18 exhibits distributions of RMSD values for comparisons of contactless probes to contact exemplars as well as between pairs of encounters acquired using the same contact device, $\mathbf{C 1}$. The $\mathbf{C 1 a}$ vs $\mathbf{C 1}$ comparisons show the lowest RMSD as well as the smallest variance. As these image pairs are acquired using the same device, such similarity in frequency content would be expected. Other comparisons are not radically disparate in median position, except for TA that spectrally is least similar to the contact exemplar, C1. As device TA attempts to "unwrap" the 3D finger, the lower sample rate 
of this extended coverage may account for the observed difference in spectral content. This is speculation, however.

\subsubsection{Correlation of SIVV Signals}

The RMSD measures the total deviation of point-wise comparison of the SIVV signals.

The Pearson product moment correlation coefficient [PEARSON] measures the parallelism between the two signals irrespective of the magnitude of the difference between them. Accordingly, we compute the correlation coefficient between s1 and s2 as

$$
r\left(\mathbf{s}_{1}, \mathbf{s}_{2}\right)=\frac{\sum_{i=1}^{n}\left(\mathbf{s}_{1}-\overline{\mathbf{s}}_{1}\right)\left(\mathbf{s}_{2}-\overline{\mathbf{s}}_{2}\right)}{\sqrt{\sum_{i=1}^{n}\left(\mathbf{s}_{1}-\overline{\mathbf{s}_{1}}\right)^{2}} \sqrt{\sum_{i=}^{n}\left(\mathbf{s}_{2}-\overline{\mathbf{s}}_{2}\right)^{2}}}
$$

where $\overline{\mathbf{s}}_{1}$ and $\overline{\mathbf{s}}_{2}$ are the arithmetic means of the two SIVV signal vectors.

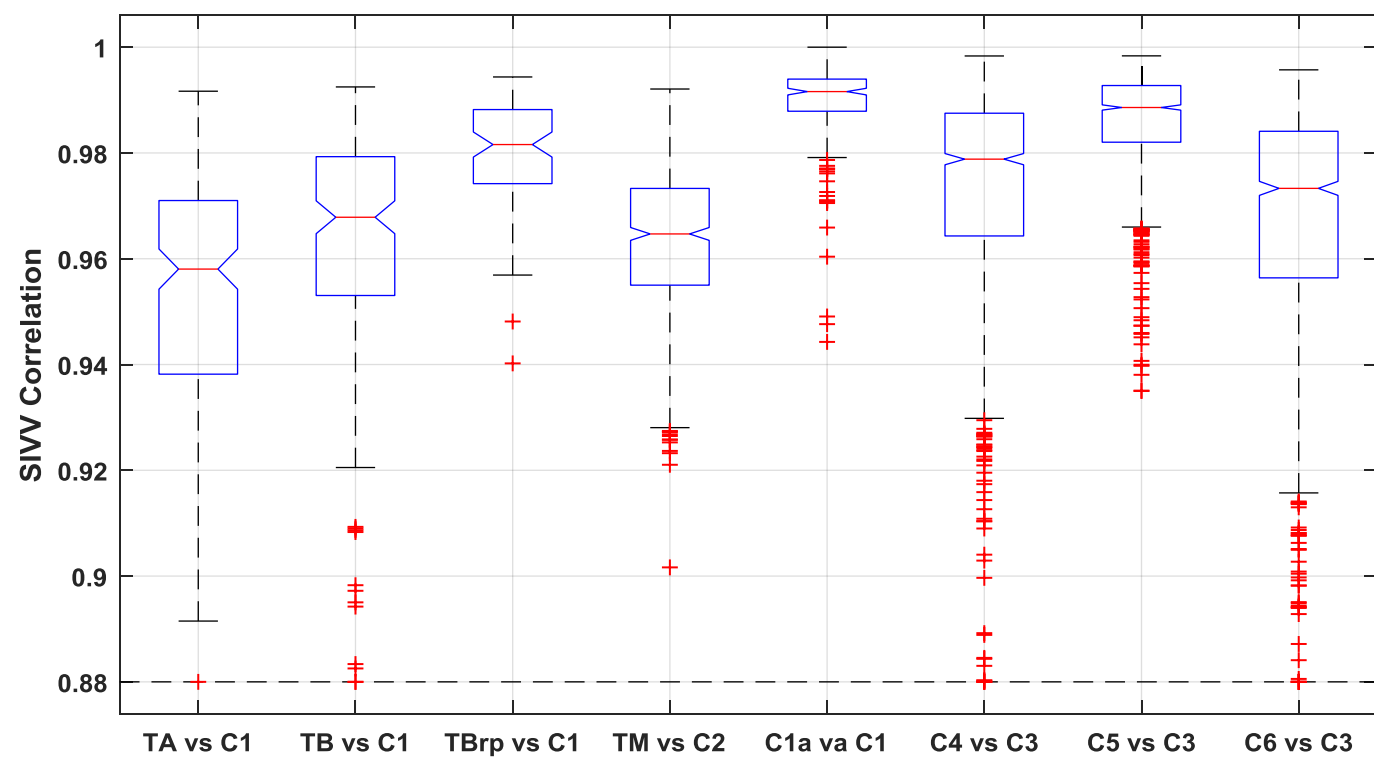

Figure 19 - SIVV correlation between spectra of corresponding contactless and contact exemplars; between two encounters from C1, and between different contact devices.

Figure 19 exhibits distributions of SIVV signal correlation coefficients for comparison of device fingerprint impressions. Again, we see the best agreement between the two C1 images, with a median value around 0.99 and small dispersion. While the contactless device image spectra show lower frequency signal correlation and broader dispersion, the median correlations are moderately high. The notable contactless performer is the reprocessed images captured by the TB device, we designate as TBrp. 


\subsubsection{Correlation of Ridge Orientation Maps}

We compare via correlation the block-wise estimates of ridge orientation. We create an orientation map of ridge orientation for each of the two overlapping regions of the registered images, I'A and I'B. For this we apply the method described in [THAI] as modified by Kovesi for a MATLAB function [KOVESI]. The method estimates the local orientation of ridges within a $7 \times$ 7-pixel block centered on each pixel of the image being processed. The output is a map of angles in radians corresponding to the size of the input image. Thus, applying the procedure to the cropped regions of overlap, I'A and I'B, we get orientation maps, OA and $\mathrm{OB}$. We then simply compute the correlation of the two orientation maps according to equation (7) above.

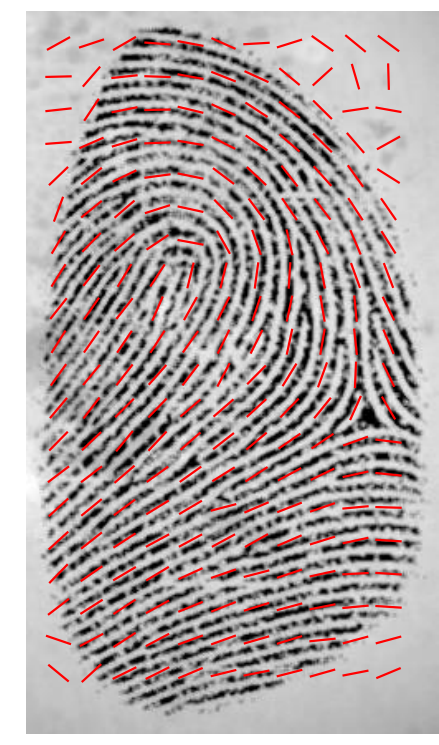

Figure 20 - Synthetic fingerprint showing vector field of estimated ridge orientations.

Figure 20 provides an example of a synthetic fingerprint overlain by a graphic depiction of the vector field of local ridge orientation. This depiction is coarse compared to the actual array of angles which includes a value computed at every pixel location of the source image. Figure 21 exhibits the distributions of 2D correlations of orientation maps for touchless captures to the corresponding control contact images and for comparisons between contact captures. The encounters from the same contact device, $\mathbf{C 1}$, of show the highest agreement in local ridge orientation, followed by comparisons of the other contact devices. Of the contact devices, the comparison of the inked cardscan to the FTIR exemplars ( $\mathbf{C 6}$ vs $\mathbf{C 3}$ ) shows the lowest ridge orientation similarity. Contactless devices, TA and TB perform similarly, followed by TBrp with overlap of confidence intervals about the medians. The mobile contactless device, TM agrees least with the ridge orientation of the contact exemplar. 


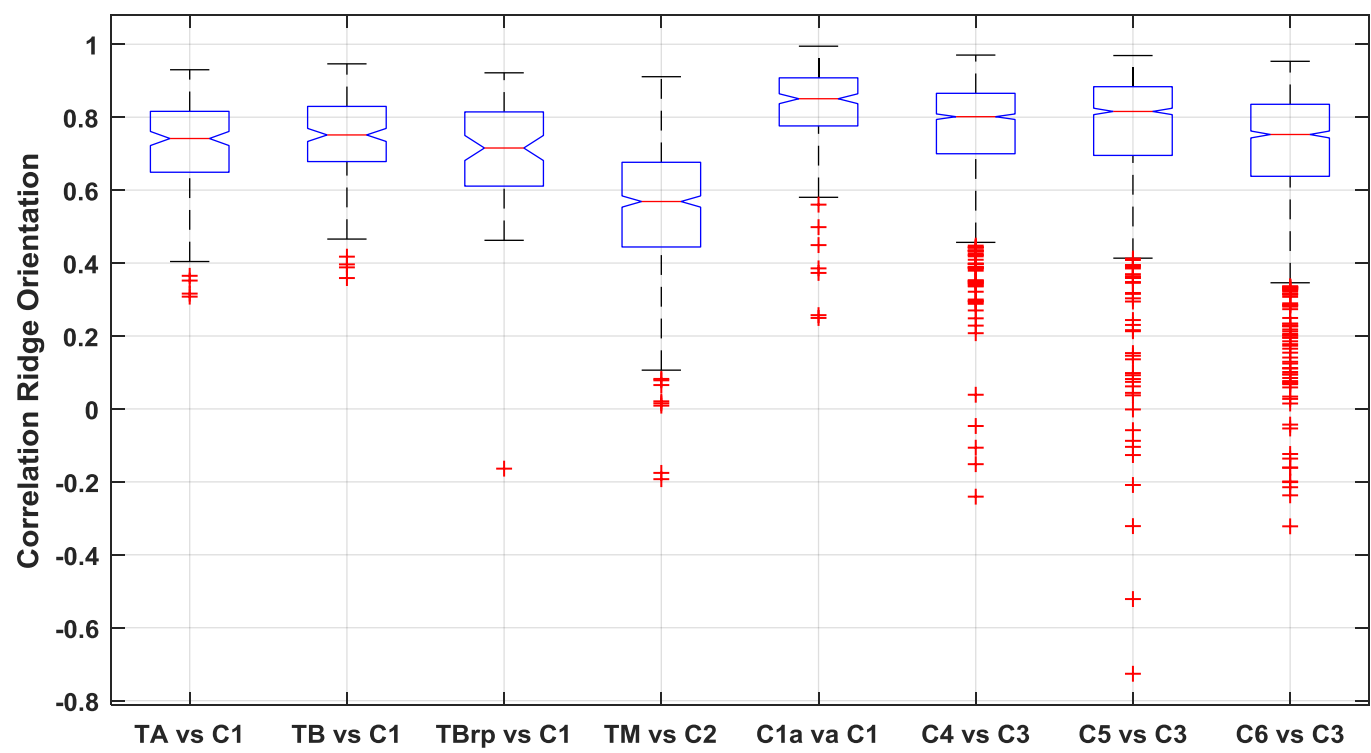

Figure 21 - Distribution of correlation of ridge orientation maps estimated for overlapping regions of fingerprints under comparison, contactless devices TA, TB, TBrp, and TM against contact exemplars and for mated contact impressions.

\subsubsection{Complex Wavelet Structural Similarity Index (CWSSIM)}

The complex wavelet structural similarity index (CWSSIM) is detailed in [SAMPAT]. It provides the means by which to compare images with respect to their locally evaluated phase differences, proposed to avoid the deficiencies of pixel intensity comparisons. Image pairs are compared on a block-by-block basis, e.g. 7 x 7 pixel samples, each block yielding a CWSSIM value and the values pooled to yield the final single value. We use a MATLAB implementation also referenced in [SAMPAT]. We remind the reader that CWSSIM comparisons are made between the overlapping regions of registered images.

Figure 22 displays the results of image comparisons via the CWSSIM method. The CWSSIM is sensitive to local image structure as sampled by local contrast and phase direction and wavelet scale. One would therefore expect the greatest values of the metric for images exhibiting similarity of local pixel organization (texture) and contrast. Thus, we see the highest values for C1a vs C1 comparisons of encounters from the same FTIR device. Other contact-to-contact comparisons exhibit lesser values as the pairs are derived from different devices, though as contact images they retain similar structure. Notably, the CWSSIM distributions among contact-to-contact are lowest for $\mathbf{C 6}$ vs $\mathbf{C 3}$ where we have inked card-scanned images compared with those from an FTIR device.

Compared to contact-to-contact comparisons, Figure 22 shows comparatively low CWSIM scores for contactless-to-contact reflective of the marked difference in character between contact exemplars and the friction ridge renderings produced by contactless devices. As we have observed, CWSSIM degrades with increasing difference in the 
image-forming process. Hence, the marked contrast between contactless and contact comparisons becomes apparent.

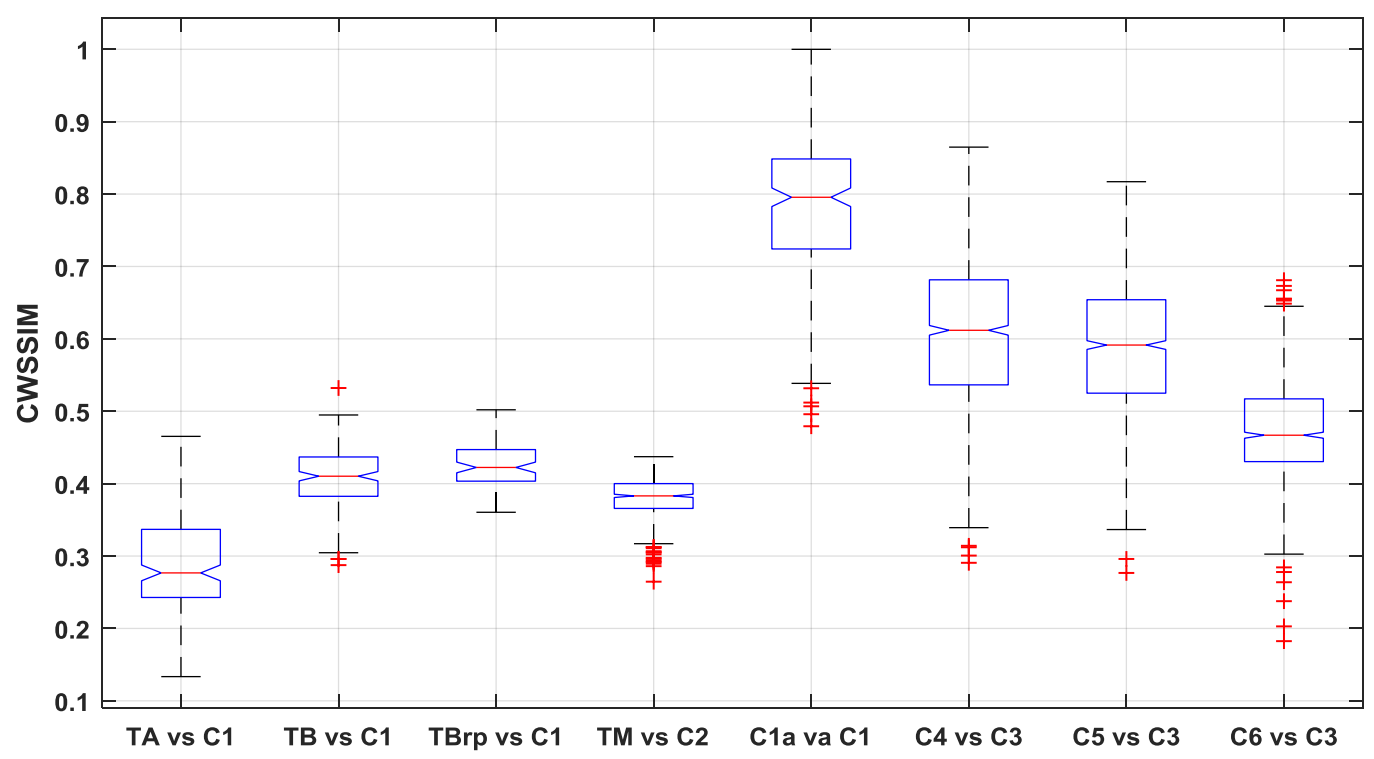

Figure 22 - Distributions of CWSSIM comparisons for contactless to contact and contact to contact devices. The CWSSIM is sensitive to differences in both contrast and local structure of images, hence the highest values for $\mathrm{C} 1$, where both images are acquired using the same FTIR device.

\subsubsection{Minutiae Comparison and Matcher Similarity}

Given that the cropped image regions under comparison are spatially registered to one another, it is possible to measure the distance by which corresponding minutiae are offset from one another. The process of image registration enables us to compare the image positions of corresponding minutiae in the registered images.

The inputs to this analysis were the registered fingerprint impressions in their entirety rather than simply the overlapping, cropped regions. Hence, this was comparable to a typical mated match scenario with the exception that the images were identical in dimension and the fingerprint impressions contained were spatially registered as described previously.

\subsubsection{Number of Corresponding Minutiae}

We first examine the number of corresponding minutiae detected with comparison between the registered device output to the mated contact control image. Distributions for each of the device comparisons are displayed in Figure 23. Contactless devices TA, TB, and TBrp display similar performance with respect to both medians and variation of distributions. Mobile contactless device, TM, shows lower numbers of minutiae in common with the $\mathbf{C 1}$ exemplars. The control contact comparison, C1a vs $\mathbf{C 1}$, shows the largest numbers of detected corresponding minutiae, consistent with other measures 
demonstrating that in fingerprint comparison, "likes prefer likes." Again, comparisons among the other contact devices reflect performance governed by the degree of similarity of image provenance. Similar devices and processing yield the most similar images.

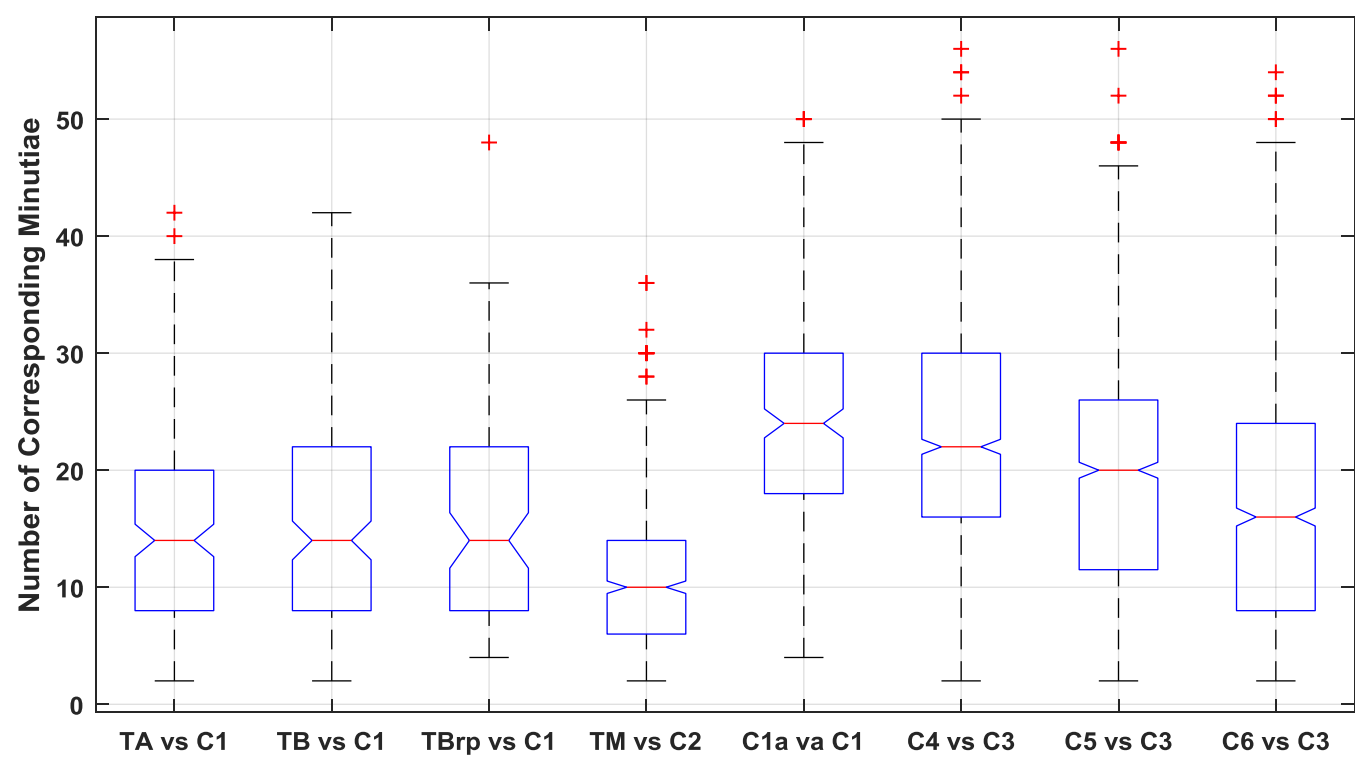

Figure 23 - Numbers of corresponding minutiae for comparison of each sensor capture with contact control impressions as determined by the state-of-the-art fingerprint feature detector.

\subsubsection{Mean Offset of Minutiae Placement}

Given minutiae correspondence between spatially registered images, we can compute the average Euclidean distance between their placement on the common coordinate system. Distributions of these measurements are displayed in Figure 24. By far the smallest mean offset is found among corresponding minutiae detected in the mated $\mathbf{C 1}$ impressions. The median value for the C1a vs $\mathbf{C 1}$ comparison is significantly lower than that of the touchless devices and its distribution shows very small variation. As with numbers of minutiae, devices TA,TB and TBrp show very similar median values and variation. The median values here are not significantly different. Device TM exhibits both greater mean minutiae offset and larger variation.

Outliers, denoted by the "+" marks, are probably the result of errors in selection of control points and consequent misregistration. Some of the images of all touchless devices and even some of the contact images challenged reliable minutiae location. Note that sample sizes for the comparisons are radically different for contactless-to-contact and contact-to-contact comparisons. 


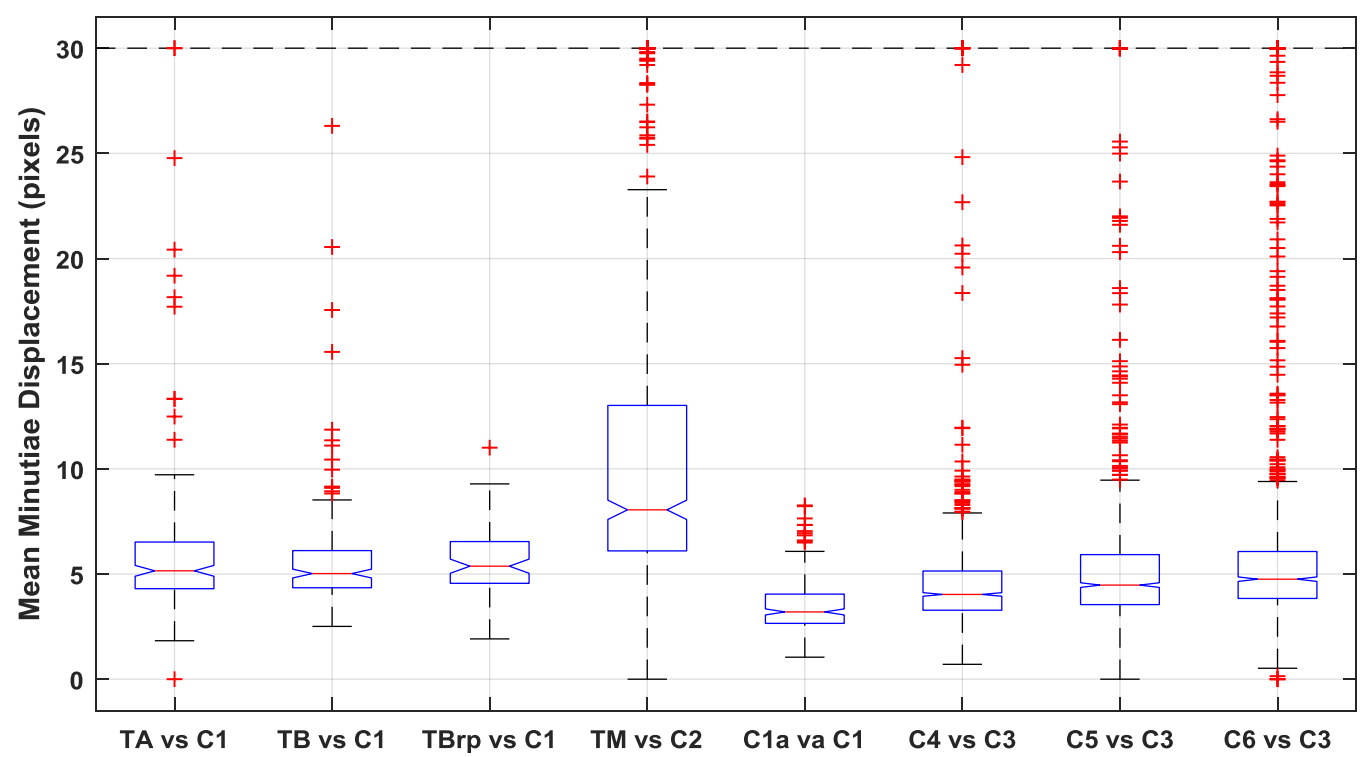

Figure 24 - Distributions of mean Euclidean distance between positions of corresponding minutiae on spatially registered fingerprint impressions captured by devices compared to control contact capture. Distance is computed for all corresponding minutiae after application of the optimized transform.

\subsubsection{Match Score}

Finally, we examine distributions of match scores computed between device captures and corresponding exemplars. This match test can be treated only as a correlation measure as a true match test examines impression relationships against a background of known nonmatch images. (A small matcher test is described later.) Regardless, we can still interpret the relative similarities of touchless to contact captured mates and that between the two contact device captures ${ }^{19}$.

Distributions of matcher similarity scores are exhibited in Figure 25. The contact-tocontact mated comparisons shows median and most of the distribution significantly higher than any of the touchless devices in mated comparisons with contact control impressions. In spite of this, however, both TA, TB, and TBrp distributions score significantly higher than the maximum similarity scores computed for known non-mated impressions. The non-mate (impostor) test consisted of 5096 pairs of known nonmates. Outliers for impostor comparison extend as high as 1000 , but the bulk of the score distribution lies below the score of 250. Smartphone application, TM, has a median

\footnotetext{
${ }^{19}$ The contactless devices used to collect data appearing in this report are under development. The analyses presented in this report reflect the state of these devices at the time the data were collected and are not reflective of current or potential future performance. The analysis of the small samples of fingerprint images are intended only to serve as the medium by which to examine and demonstrate the behavior of candidate measurements and analysis procedures being developed by this NIST project.
} 
match score well over the imposter distribution, but its distribution extends dangerously into the imposter realm, indicating that it would suffer significant match errors in a matcher test. C1 matches among paired encounters are virtually all separated from the impostor score distribution, as are most of the scores for contact-to-contact matches. We should note in the interest of fairness that registration was visually verified for contactless-to-contact comparisons as the numbers were relatively small. Contact-tocontact comparisons involving the NIJ images from C3, C4, C5, and C6 were too great in number for practical screening of cases of failed registration. Such cases remain few compared to the $1100-1200$ cases included, but noise is present in these data, reflected in extension of the boxplot whiskers toward lower score values.

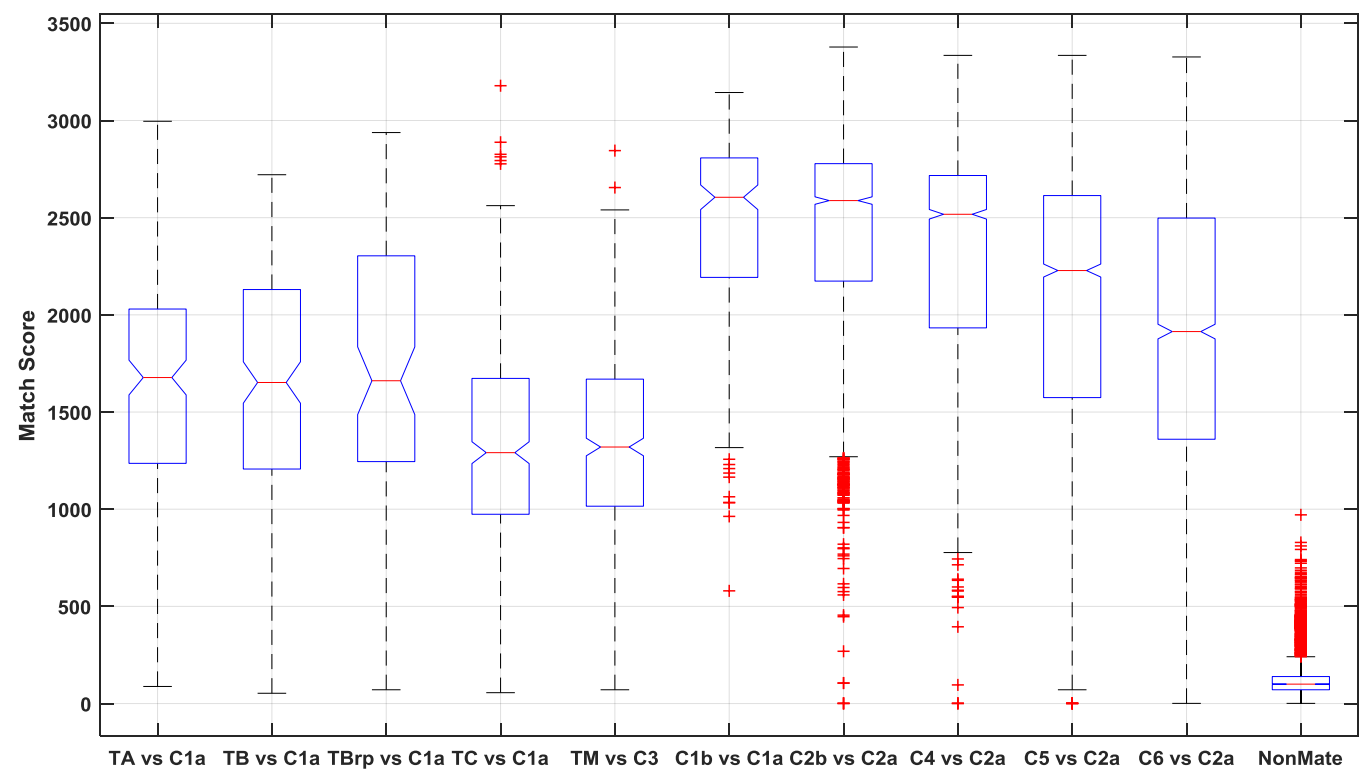

Figure 25 - Distributions of matcher similarity scores of device captures and contact control impressions. A distribution of $\mathbf{5 0 9 6}$ non-mate scores is included for reference (see far right).

\subsubsection{Matcher Test}

We examine distributions of match scores from a state-of-the-art operational biometric matcher. The matcher was applied to the images of contactless devices, TA, TB, TBrp, and $\mathbf{C 1}$, each compared to the control exemplars, C1a.

Although most operational submissions are multi-finger submissions (e.g., ten-print, slap4), single-finger submissions are not unknown. Subject identification is typically the goal of this mode, to which end fusion algorithms are applied to scores from multiple fingers. These algorithms combine, or fuse, the match scores of individual fingers to develop a match score reflecting the similarity of the fingerprint images of the subject (probe) to corresponding images of known individuals in the fingerprint image repository (gallery). 
Due to the small size of our contactless subject pool, we have avoided for the present attempting to perform "subject matching", choosing instead to examine single finger match performance. Subject matching is reserved for follow-on work for which we are able to collect images from a larger pool of individuals. In this planned work, we will examine various multiple-finger performance as well as that of single finger comparisons.

From these raw scores, we compute two primary error rates - the False Match Rate (FMR), and the False Non-Match Rate (FNMR) [ISO3]. Neither of these rates are absolute; rather, both are parametric functions of the reported match score. A mathematician might prefer the clarity of using the notation $\mathrm{FMR}_{(t m s)}$ and $\mathrm{FNMR}_{(t m s)}$, where tms is the independent parameter threshold match score. In plain English, $\operatorname{FMR}_{(t m s)}$ is the probability that for a random member (in the present case, each 'member' is a fingerprint image) of the probe set and a random non-mated (i.e., coming from a different subject) image of the gallery, the reported similarity score will be equal to or greater than the parameter tms. Likewise, $\mathrm{FNMR}_{(t m s)}$ is the probability that for a random image of the probe set and a mated (i.e., coming from the same subject) image of the gallery, the reported similarity score will be less than the parameter tms.

The matcher testing procedure and scoring is detailed in Appendix B. The match performance is summarized by a Receiver Operating Characteristic (ROC) curve or plot in which we plot TMR against FMR, where The True Match Rate (TMR) is simply (1FNMR).

The ROC plot shows the effect, for a given matcher and a given set of data, of choosing a particular operating threshold: if we choose a threshold $x$, then we have simultaneously chosen $\operatorname{FMR}_{(x)}$ and $\operatorname{TMR}_{(x)}$. What's really convenient is that we needn't specify $x$, but rather can choose a value for FMR and immediately see the corresponding value of TMR, or conversely can choose a value for TMR and immediately see the corresponding value of FMR. 


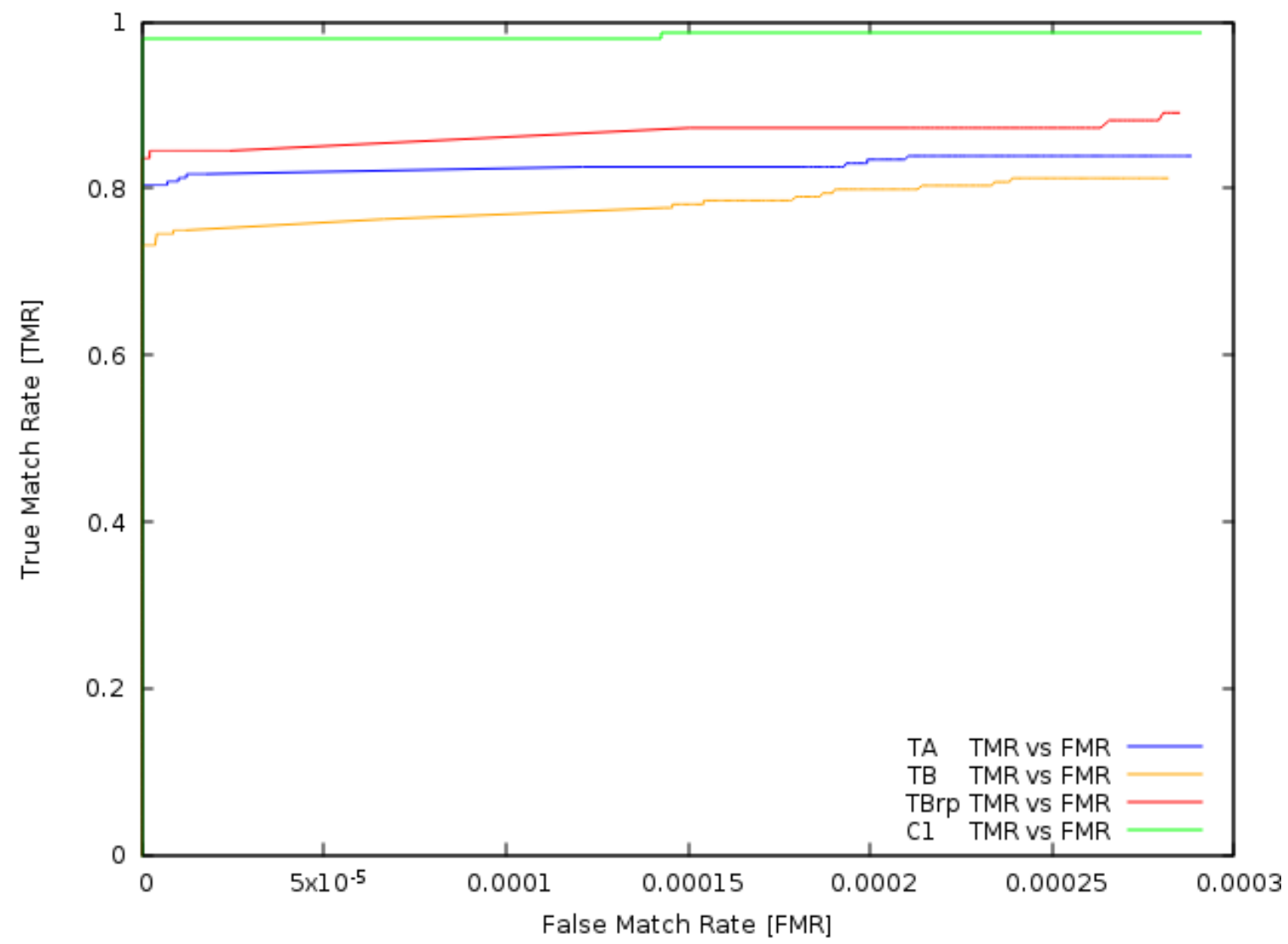

Figure 26 - Receiver Operating Characteristic (ROC) graph for the small matcher test showing the True Match Rate as a function of False Match Rate.

Note that because of the small sample sizes, and the rarity of false matches with the chosen matcher, that we cannot state with certainty how low FMR can be. However, we have enough data to be reasonably confident in our findings about TMR, and thus about FNMR. (FNMR $=1-$ TMR.) We have shown this in Figure 26.

\subsection{Discussion of Quality and Interoperability}

Insofar as the ultimate test of interoperability is the ability to match the touchless fingerprint impression to a legacy contact impression, we find the match score itself to be most significant. At least two of the touchless devices exhibit scores well above an impostor baseline for mated matches to legacy device control images. Examination of minutiae number, displacement, and angle differences show their respective contribution to the pattern observed in match scores among the four devices ${ }^{20}$. The correlation of ridge

\footnotetext{
20 The contactless devices used to collect data appearing in this report are under development. The analyses presented in this report reflect the state of these devices at the time the data were collected and are not reflective of current or potential future performance. The analysis of the small samples of fingerprint images are intended only to serve as the medium by which to examine and demonstrate the behavior of candidate measurements and analysis procedures being developed by this NIST project.
} 
orientation maps shows a pattern similar to that for match scores and minutiae measurements.

As a single image metric, NFIQ2 combines a large number of measurements into a composite score. Having visually examined all the impressions of the present study, we can assert that the elevation of $\mathbf{T B}$ above even the $\mathbf{C 1}$ is likely spurious. While most of the TB images showed sharp edges and low noise, the result of most in the sample being binary. Even in cases where the ridge structure was severely broken, the segments appeared as well-defined line segments. What is surprising in this regard is that the minutiae comparison and match scores remained relatively good for this device. Also, somewhat surprising is the minutiae and match performance of TA given that its impressions suffered from low contrast and often difficult to discern ridge structure amidst highly textured, noisy overall appearance. That we find reasonably good performance for these devices may testify to the robustness of the matcher used or perhaps any of a number of modern matchers.

An observation worthy of note is that all of the contactless devices exhibited varying degrees of polarity reversal in the rendering of the ridge structure. Because of the vagaries of lighting angle across the surface, a ridge crest could either be locally brighter or darker than adjacent furrows or even in between. Often the brightest and darkest regions of a ridge are not actually coincident with ridge crest or valley floor but with opposing flanks of the ridge where one side of the ridge receives light and the opposite side is in shadow.

This polarity reversal effect is visible in the photographic representation where such is available, but is lost completely in the grayscale or binary rendering of the photograph. Accordingly, it was not uncommon in comparing touchless prints with mates captured using the FTIR technology to find it difficult to find corresponding minutiae when looking specifically for either ridge endings or ridge bifurcations. Instead, a ridge ending in the contact exemplar sometimes did not appear similarly positioned in the touchless print. Yet the touchless print displayed a bifurcation in the corresponding position. We found that the minutiae correlator and match software used for measurements reported in Section 5.3.6 did not report minutiae type - only $x, y$ coordinate and angle. However, we do not know if the matcher of the correspondence tool considered minutia type, but simply did not report it, or if it used only the feature position in its analysis, i.e. treating bifurcations and ridge endings as interchangeable.

More study of how matchers handle minutiae type might relieve some of the concern over polarity reversal. Whereas the polarity reversal problem of touchless sensors would significantly inhibit human fingerprint comparison and evidentiary value of contactless fingerprints, the problem may be overestimated in importance where machine matching is concerned. The two matchers used in the present study were not adjusted in any way for contactless-to-contact comparison, but reducing the weighting on minutiae type might enhance match performance of contactless fingerprints. 
Nevertheless, we recommend that the photographic original be retained if possible. In our experience, the photograph often provides the least ambiguous representation of the touchless fingerprint impression. Moreover, advances in fingerprint rendering techniques may enable reprocessing of the retained raw images to improve the quality of the rendered patterns, and hence their interoperability with legacy images. 


\section{Image Anomalies}

\subsection{Polarity Inversion}

Any algorithm(s) used to convert a photograph of the finger into a grayscale representation must make assumptions as to how to interpret the image features as either ridges or furrows. In the contact fingerprint ${ }^{21}$, there is no such decision because local topographic highs will always be dark, roughly in proportion to pressure or the degree of contact, and the furrows where no or little contact is made will always be lighter in tone. For contactless capture, reflection from the light source is frequently greatest from ridge crests, though not always and not uniformly. Depending on the local angle of illumination, flanks of ridges may be bright with shadows on the lee flank of the ridge. Regardless, the developer of the rendering software must decide on how to process the images relative to interpretation of grayscale as grayscale applies to fingerprint structure.

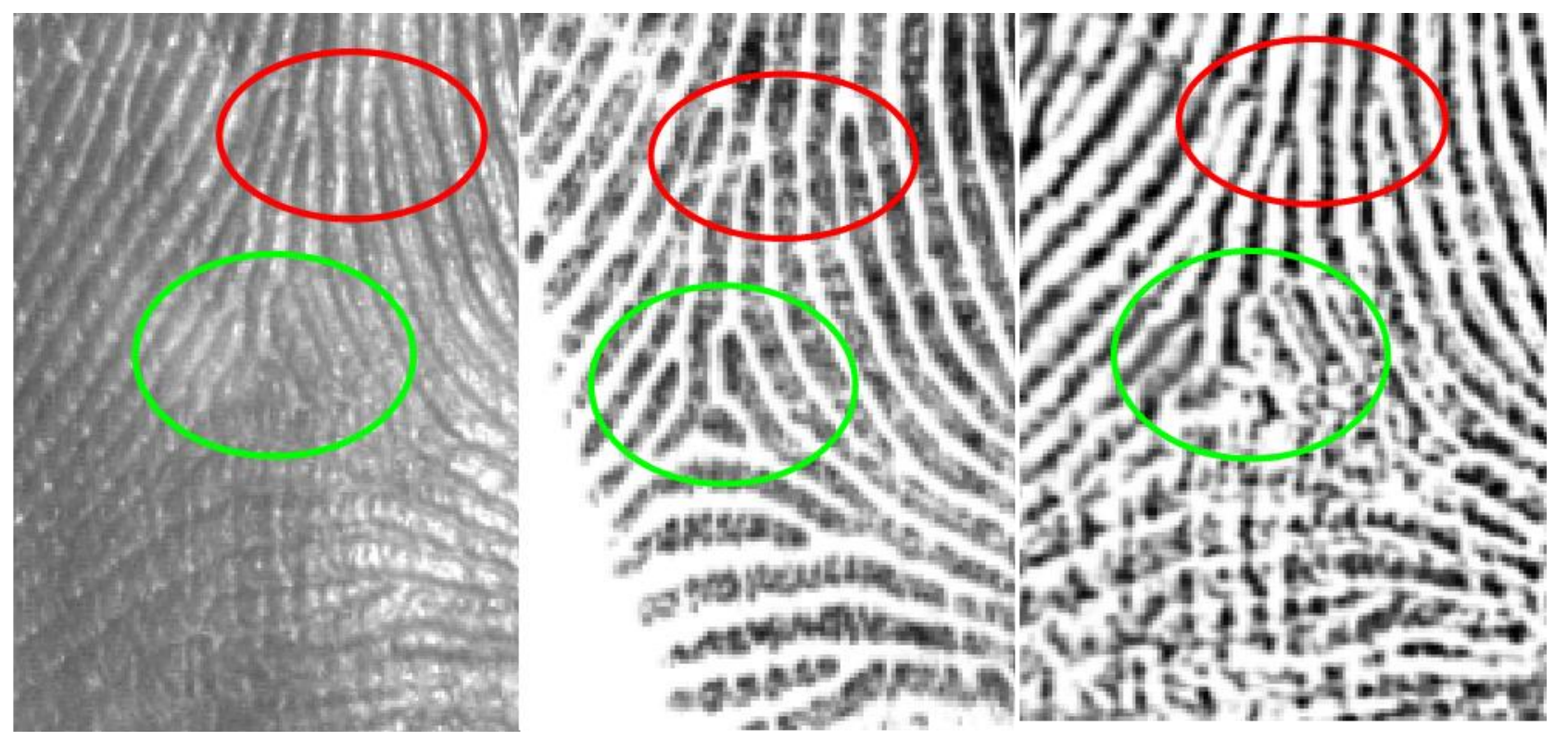

Figure 27 - As designated by the red ellipses, note the three ridge endings in the photograph (left) captured by a contactless device and the corresponding features in the livescan capture (center). In the grayscale rendering of the contactless capture (right), the ridge endings become bifurcations (furrow endings). The delta feature designated by green ellipses is formed by ridges in the photo and livescan images, but by a furrow, incorrectly toned dark in the contactless print.

In many instances we observed, the processing calls for inverting the grayscale, i.e. swapping bright and dark pixel values. If ridge crests were bright in the raw photograph, they become dark in the inverted version and may be further processed and enhanced to render them as the ridges in the final processed output. Failures of this assumption on the global or local level can create problems.

${ }^{21}$ Fingerprints depicted in this document were collected under an approved NIST Human Subjects Protection Office (HSPO) protocol, with permission to publish granted by donor. 
Figure 27 demonstrates the problem known as contrast reversal or polarity inversion in which ridges are mispresented as furrows and vice versa in rendering a grayscale fingerprint from the original captured photograph. We see the original photograph of a small sample of a contactless capture displayed on the left. Immediately to the right of this is roughly the same area of the friction ridge pattern sampled from a contact (FTIR) capture of the same finger. The red ellipses mark three ridge endings seen in the rendered contactless fingerprint on the right to appear as bifurcations. The likely source of the error becomes apparent as we examine Figure 28. We have converted the color photograph to grayscale ${ }^{22}$ inverted the grayscale of the photograph and see that this operation $^{23}$ alone transforms the ridge endings in the photograph into bifurcations, the original lighter furrows now rendered as ridges.

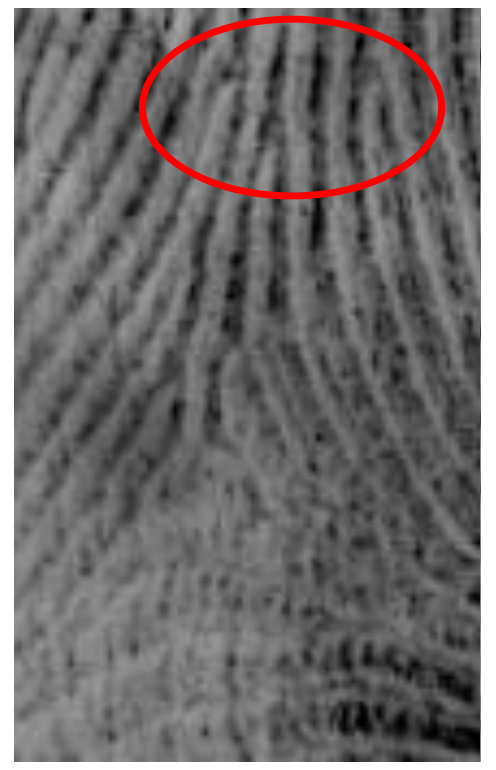

Figure 28-Grayscale inversion of the original photograph depicted in Figure 27 illustrating that the furrows have been misrepresented as ridges giving us bifurcations rather than ridge endings.

\subsection{Poor Focus}

Contactless acquisition does not constrain the position of the fingers during capture, nor does it involve pressure to flatten the 3D presentation of the friction ridge pattern as we find in contact capture. As the capture is photographic, any portion of the finger outside the depth of field of the lens due to out-of-plane presentation will be blurred. Issues with non-uniform illumination might also be involved. Rendering algorithms are unable to recover ridge structure in such poor focus regions.

\footnotetext{
22 The RGB image may be converted to 8-bit grayscale forming a weighted sum of the $R, G$, and $B$ components: Grayscale $=0.2989 * \mathrm{R}+0.5870 * \mathrm{G}+0.1140 * \mathrm{~B}$

${ }^{23}$ For 8-bit images having pixel values 0 for black and 255 for white, multiply each pixel value by -1 and add 255 .
} 
Figure 29 (left) exhibits a portion of the photographic capture of a fingerprint ${ }^{24}$. The contact capture of the region depicted appears in the center position, displaying the ridge pattern quite clearly. The rendered output extracted from the photographic original is shown on the right, illustrating the breakup of the ridge pattern due to the poor focus.

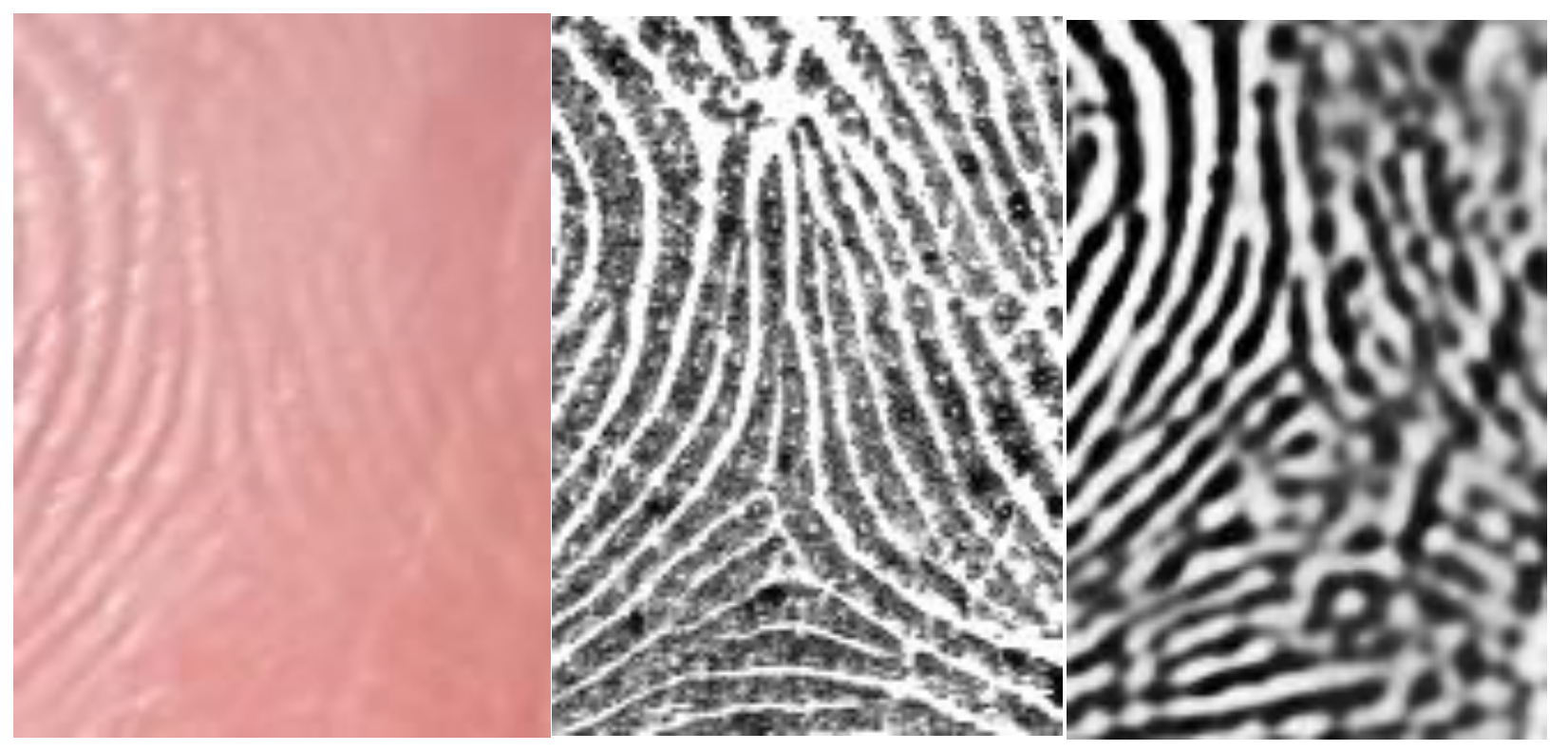

Figure 29 - Contactless photograph (left) shows a portion of a photographic capture of a finger. The contact capture of the friction ridge region (center) is crisply rendered to the point of showing level 3 features such as pores. The poor focus has inhibited rendering of the output grayscale fingerprint as seen in the rightmost image.

\subsection{Skin Irregularities}

Skin irregularities such as wrinkles, scars, and cracks can degrade even contact captures of fingerprints. With contact capture, he impact of these features is much reduced. The pressure onto the contact flattens mush of the 3D relief of such features. While such features cross-cut the ridge pattern, sometimes to an extreme degree, the features appear as breaks in the pattern, without adding additional structure.

Figure 30 illustrates how skin irregularities, somewhat disruptive for contact capture become virtual chasms in a contactless capture as modeling of the $3 \mathrm{D}$ features by illumination and the rendering processes greatly increase the prominence of such features and their impact on the friction ridge pattern.

\footnotetext{
${ }^{24}$ Fingerprints depicted in this document were collected under an approved NIST Human Subjects Protection Office (HSPO) protocol, with permission to publish granted by donor.
} 

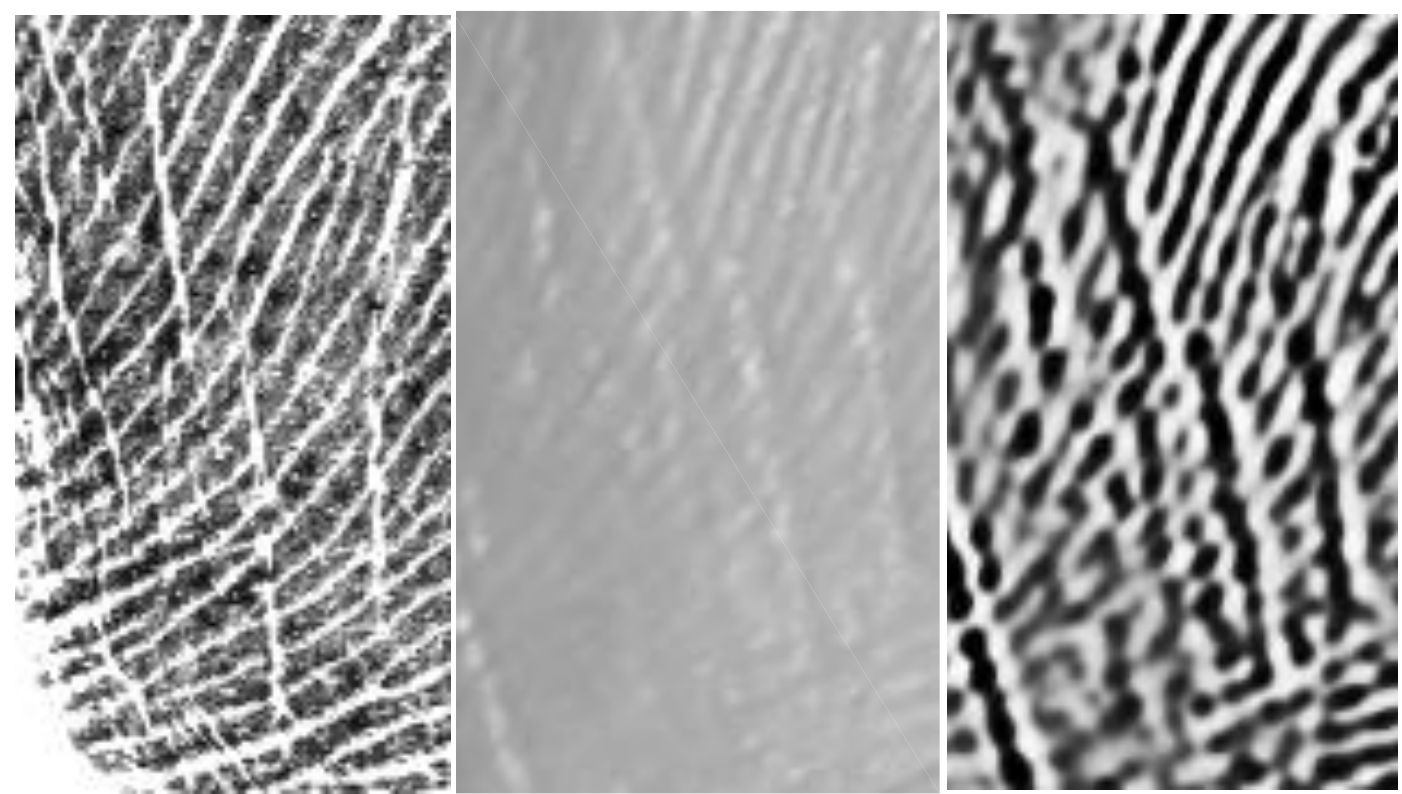

Figure 30 - The contact capture on the left exhibits skin irregularities that interrupt the ridge structure. These features are visible in the photographic capture (center) as grooves, with the 3D relief emphasized by the showing proximal to the illumination source and highlights on the distal sides of the grooves receiving direct lighting. The grayscale rendering on the right shows the grooves becoming major structural features that compete in significant way with the friction ridge structure. Polarity reversal is also evident as the illuminated flanks of the grooves are rendered dark and the shadows rendered bright. 


\section{Test Artifacts}

The analysis presented above involved collecting fingerprints from human volunteers. For device testing, relying on human volunteers poses challenges in protecting their privacy as well in ensuring the consistency of tests. Thus, we have developed a suite of artificial test artifacts that, when suitably calibrated, can be replicated and used for device testing in a repeatable fashion.

Appendix $\mathrm{C}$ describes efforts to develop artificial test artifacts, including images from the current suite of laser-engraved fingerprints. These fingerprints are sampled from a new NIST database of rolled fingerprint impressions from subjects known to be deceased, hence removing the hazard of compromising the biometric identify of living human subjects.

\subsection{Fingerprint Targets}

In addition to avoiding the privacy concerns related to using fingerprints from living human subjects, static fingerprint patterns engraved onto the surface of a relatively hard material, such as acrylic, also provide an opportunity to examine how accurately a particular fingerprint acquisition device reproduces the physical pattern in the imagery resulting from acquisition and any processing applied.

Many of the tests described in the PIV and Appendix F specifications utilize various static patterns to measure an acquisition system's ability to accurately reproduce these patterns in their respective resulting imagery [EBTS, NILL]. We have replicated and extended this approach in the design of a suite of 3D acrylic fingerprint targets with static fingerprint patterns for use with contactless fingerprint acquisition systems. Appendix C describes the design of these targets, a set of which are pictured below in Figure 31.

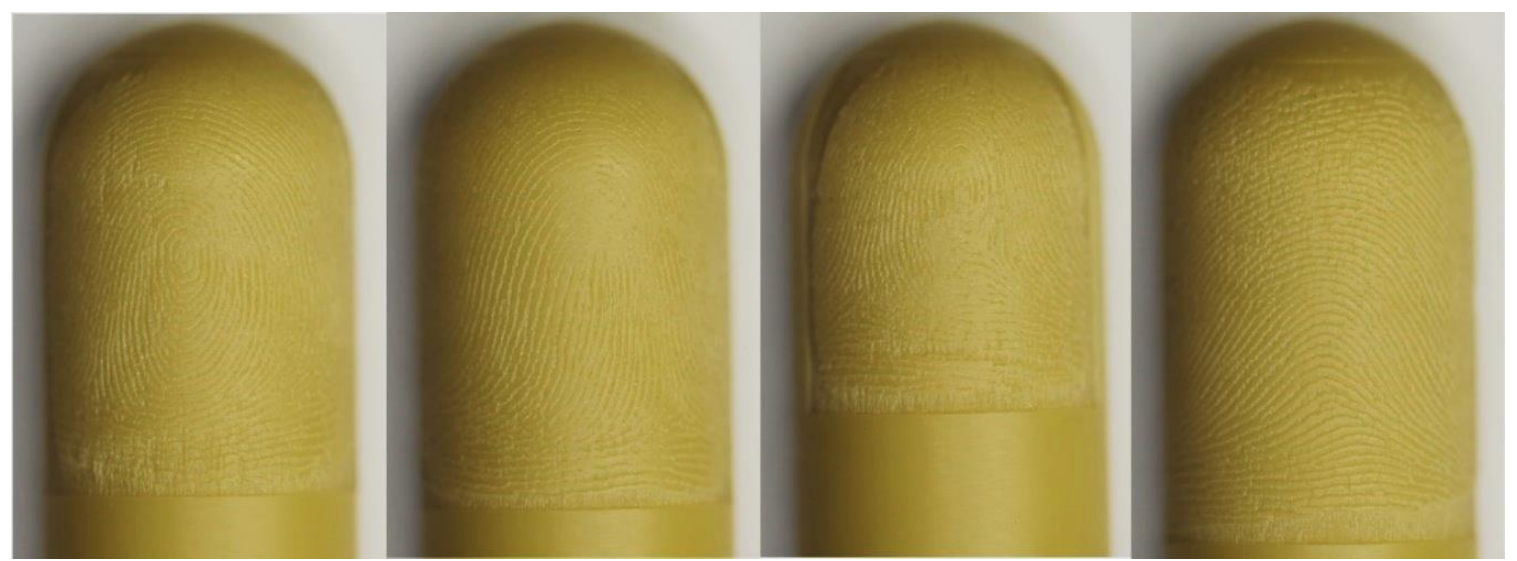

Figure 31 - Fingerprint Target Set

These targets can be captured on both legacy contact and contactless acquisition systems and the results can be evaluated using some of the metrics defined in Section 5. A selection of sample captures is shown in Section 7.4. 


\subsection{Ronchi Targets}

As discussed in Section 3.2.1 above, a 2D Ronchi ruling pattern has been employed in the Appendix F and PIV IQS in order to measure several aspects of images acquired on contact devices. However, a "flat" Ronchi pattern, bearing no relief, presents a departure from the capture modality of a contactless fingerprint acquisition system in that the presence of fingerprint ridges in a photograph of a finger is a function of light and shadow resulting from the relief of ridges and furrows on the surface of a finger (or other object) as opposed to the contrast of black and white bars on a flat surface.

In another effort to replicate and extend the concept of using Ronchi patterns as defined in Appendix F and PIV IQS, we developed a set of 3D Ronchi targets as described in Appendix C and pictured below in Figure 32.

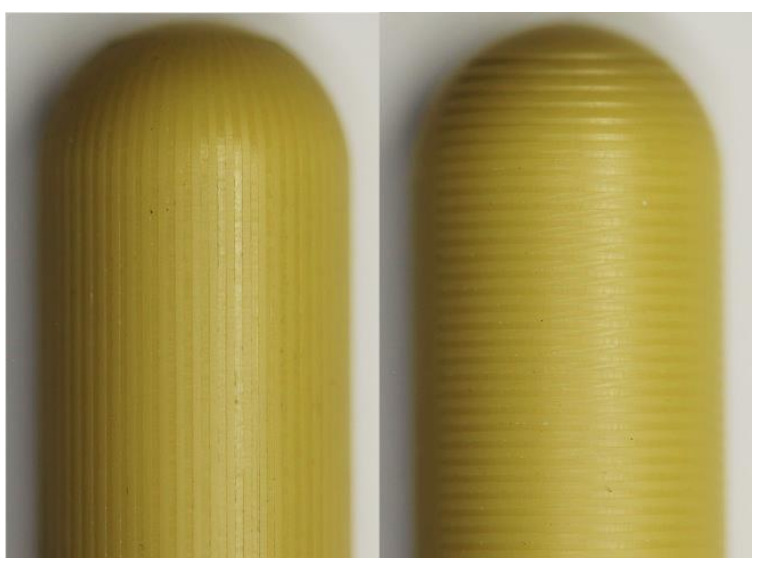

Figure 32 - Ronchi Target Set

As with the fingerprint targets, the Ronchi targets can be captured on both contact and contactless systems, though with some difficulty in operational mode on some devices most likely due to their dissimilarity to fingerprint patterns. A selection of sample captures is shown below in Section 7.4.

\subsection{Concentric Circle Target}

After observing some of the image anomalies unique to contactless fingerprint acquisition, as described in Section 6, a concentric circle target (shown in Figure 33) was designed to enable the observation, detection, and measurement of reveal polarity reversals, ridge continuity, and geometric distortion with a set of 25 concentric circles. The thickness and spacing of these circles approximates the thickness and spacing of fingerprint ridges (see Appendix C). Captures exhibiting polarity reversals or other geometric and continuity anomalies are readily visible in the apparent departure from smooth circular shapes in the resulting images. 


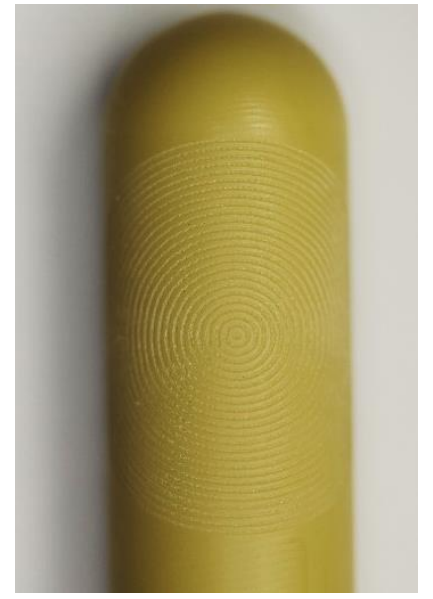

Figure 33 - Concentric Circle Target

Capturing the current concentric circle target using contactless fingerprint acquisition systems is more difficult and prone to (capture) failure than the fingerprint or Ronchi targets, but with more refinement and development this design may prove to be crucial in measuring and detecting certain anomalies in contactless fingerprint imagery. A limited selection of sample captures of this target in shown in Section 7.4.

\subsection{Sample Captures}

The figures below show sample captures taken across a set of contactless acquisition devices using the various target designs described above.
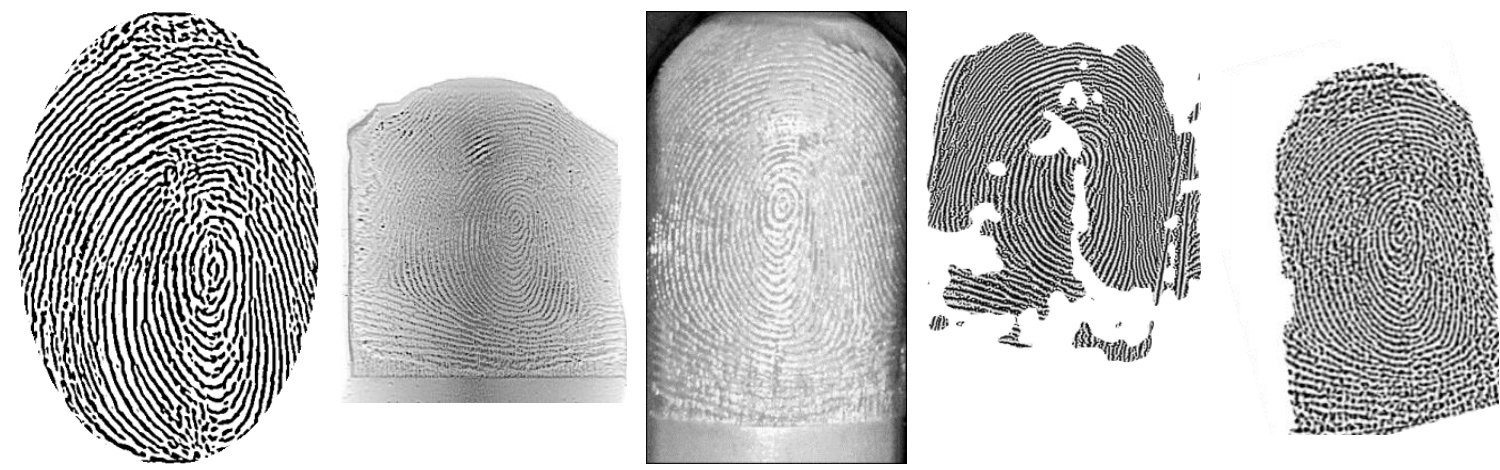

Figure 34 - Contactless Captures of Fingerprint Target

Figure 34 shows sample captures of one fingerprint ${ }^{25}$ target out of the set of four. This provides an overview of the differences in the representation of an otherwise static fingerprint pattern across a set of five contactless devices of various designs. As shown, some images appear to be more extensively processed, while others appear to be more similar to an unprocessed photo of the target.

${ }^{25}$ Fingerprints depicted in this document were collected under an approved NIST Human Subjects Protection Office (HSPO) protocol, with permission to publish granted by donor. 
Overall, the pattern is rendered relatively well using each of the devices however a few potential problem areas exist as well. For example, in one of the images above a nontrivial portion of the fingerprint pattern is missing.

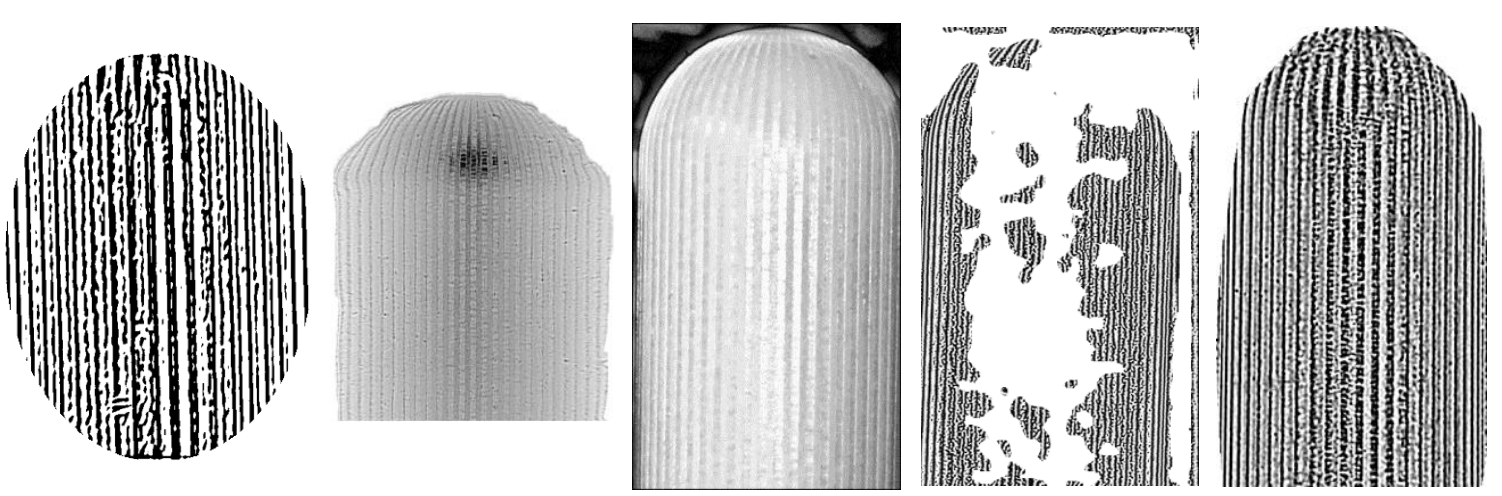

Figure 35 - Contactless Captures of Vertical Ronchi Target

Figure 35 shows an example of the images produced by a set of five contactless acquisition devices using the vertical Ronchi target. These samples show the same relative levels of processing observed in the fingerprint target images shown in Figure 34, but also reveal some potential discrepancies in the reproduction of the Ronchi pattern.

For example, some images show varying levels of sharpness and clarity in the transition from light to dark bars. Some images also appear to depict bars of varying width or spacing, as shown in Figure 35.
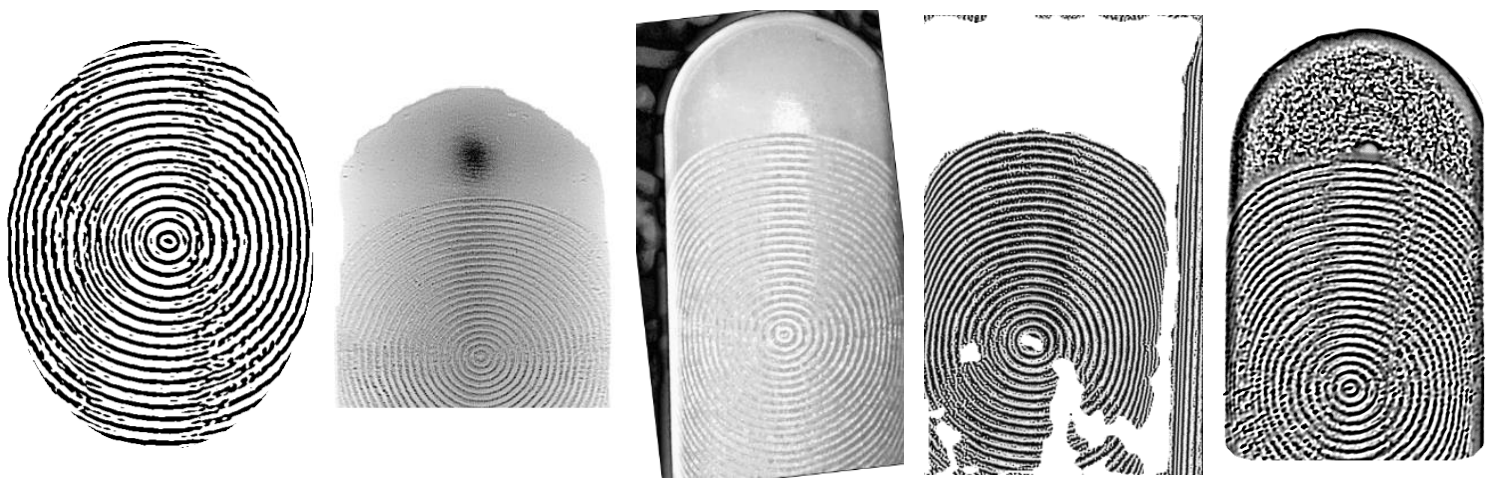

Figure 36 - Contactless Captures of Concentric Circle Target

Figure 36 shows a sampling of images resulting from the acquisition of the concentric circle target on a set of five contactless devices. These samples show an overall high fidelity to the original pattern; however, some images also reveal what may be evidence of a contrast or polarity reversal (as described above in Section 6.1). 


\subsection{Future Work}

Further investigation and testing is required to refine the measurement strategies and methods applied to these targets in order to enable semi-automated measurement and define a range of acceptable fidelity for each target. Refinements to the design of some targets may also allow for an improvement in the ease with which we are able to capture the targets on some devices.

The physical design of the various targets appears to allow for capture on all of the contactless acquisition devices tested, however there are some challenges in capturing these targets properly using legacy contact acquisition devices. Attempts to capture the targets using an FTIR device show that the design of these targets does not preclude interacting with a contact device. However, the software required for rendering an interaction with the device into an image may need to be modified in order to allow for the proper the imaging of a static, inflexible object. The ability to accurately image these targets on a legacy contact device would allow direct comparison of contact to contactless performance using these targets and also apply some of the metrics described in Section 5.

In addition to direct comparisons with contact imagery, the ability to automate the measurement of certain features in contactless images of the targets is also being explored. Currently, measurements such as counting the number of pixels comprising the width of a bar in an image of one of the Ronchi targets are made manually. The goal is to automate this process so that such measurements can be made more quickly, accurately, and reliably.

We will continue to pursue the refinement of the design of these targets as well as methods for their capture and measurement using both contact and contactless acquisition devices. 


\section{References}

\begin{tabular}{|c|c|}
\hline BOULT & $\begin{array}{l}\text { T. Boult. Beyond Image Quality: Failure Analysis from Similarity Surface Techniques. } \\
\text { presentation to NIST Biometric Quality Workshop, Gaithersburg, MD, March } 2006 .\end{array}$ \\
\hline EBTS & $\begin{array}{l}\text { Electronic Biometric Transmission Specification (EBTS), Department of Justice Federal } \\
\text { Bureau of Investigation (FBI), Criminal Justice Information Services (CJIS), July 2, 2013, } \\
\text { (Appendix F), } \\
\text { https://www.fbibiospecs.cjis.gov/Document/Get?fileName=Master\%20EBTS\%20v10\%20- } \\
\text { \%20FINAL\%2020130702.pdf }\end{array}$ \\
\hline ISO1 & $\begin{array}{l}\text { ISO/IEC. TR 29794-4:2010. Information technology biometric sample quality part } 4 \text { : } \\
\text { Finger image data. Technical report, JTC } 1 / \mathrm{SC} 37,2010 .\end{array}$ \\
\hline ISO2 & $\begin{array}{l}\text { ISO/IEC. IS 29794-1:2016. Information technology biometric sample quality part 1: } \\
\text { Framework. ISO Standard, January } 2016 .\end{array}$ \\
\hline ISO3 & $\begin{array}{l}\text { ISO/IEC 19795-1:2006(E). Information technology — Biometric performance testing and } \\
\text { reporting - Part 1: Principles and framework, April } 2006 .\end{array}$ \\
\hline KOVESI & $\begin{array}{l}\text { Peter Kovesi. MATLAB and Octave Functions for Computer Vision and Image } \\
\text { Processing. } \\
\text { Available from: <http://www.peterkovesi.com/matlabfns/> }\end{array}$ \\
\hline MATLAB & $\begin{array}{l}\text { MATLAB and Image Processing Toolbox Release R2017a, The MathWorks, Inc., Natick, } \\
\text { Massachusetts, United States. }\end{array}$ \\
\hline NIJ1 & $\begin{array}{l}\text { Ericson, L. and Shine, S. Evaluation of Contactless versus Contact Fingerprint Data. Phase } \\
2 \text { (version 1.1), DOJ Office of Justice Programs, National Institute of Justice, Sensor, } \\
\text { Surveillance, and Biometric Technologies (SSBT) Center of Excellence (CoE), (Prepared } \\
\text { by ManTech International Corporation, Fairmont, WV, May 4, } 2015 .\end{array}$ \\
\hline NIJ2 & $\begin{array}{l}\text { National Institute of Justice, Faster and Better Fingerprinting: The Fast Capture Initiative, } \\
\text { 2007, https://www.nij.gov/topics/technology/biometrics/pages/fast-capture-initiative.aspx, } \\
\text { (Retrieved 06/14/2018) }\end{array}$ \\
\hline NFIQ & $\begin{array}{l}\text { Tabassi, E. Development of NFIQ } 2.0 \text { https://www.nist.gov/services- } \\
\text { resources/software/development-nfiq- } 20\end{array}$ \\
\hline NILL & $\begin{array}{l}\text { Nill, N. Test Procedures for Verifying Image Quality Requirements for Personal Identity } \\
\text { Verification (PIV) Single Finger Capture Devices, MITRE TECHNICAL REPORT, } \\
\text { MTR060170R5, The Mitre Corporation, Bedford, MA, December 2006. } \\
\text { https://www.mitre.org/publications/technical-papers/test-procedures-for-verifying-image- } \\
\text { quality-requirements-for-personal-identity-verification-piv-single-finger-capture-devices }\end{array}$ \\
\hline NIST1 & $\begin{array}{l}\text { Libert, J., Grantham, J., Orandi, S. A 1D Spectral Image Validation/Verification Metric } \\
\text { for Fingerprints, NIST Interagency Report 7599, August, } 2009 \\
\text { http://ws680.nist.gov/publication/get_pdf.cfm?pub_id=903078 }\end{array}$ \\
\hline NIST2 & $\begin{array}{l}\text { "NIST Biometric Image Software". http://Fingerprint.nist.gov/NFIS/. (Retrieved 2011-01- } \\
\text { 12.) }\end{array}$ \\
\hline NIST3 & $\begin{array}{l}\text { Furman, S., Stanton, B., Theofanos, M., Libert, J.M., and Grantham, J. Contactless } \\
\text { Fingerprint Devices Usability Test, NIST Interagency Report 8171, March, 2017, } \\
\text { http://nvlpubs.nist.gov/nistpubs/ir/2017/NIST.IR.8171.pdf }\end{array}$ \\
\hline
\end{tabular}




\begin{tabular}{|c|c|}
\hline NIST4 & $\begin{array}{l}\text { Watson, C., Fiumara, G., Tabassi, E., Cheng, SL, Flanagan, P, Solomon, W. Fingerprint } \\
\text { Vendor Technology Evaluation, NIST Interagency Report 8034, December } 2014 .\end{array}$ \\
\hline NIST5 & $\begin{array}{l}\text { G. Fiumara, P. Flanagan, J. Grantham, B. Bandini, K. Ko, and J. Libert, NIST Special } \\
\text { Database 300: Uncompressed Plain and Rolled Images from Fingerprint Cards, NIST } \\
\text { Technical Note 1993, June 2018, https://dx.doi.org/10.6028/NIST.TN.1993 }\end{array}$ \\
\hline NIST6 & $\begin{array}{l}\text { Fiumara G, Flanagan P, Schwarz M, Tabassi E, Boehnen C, NIST Special Database 301: } \\
\text { Nail to Nail Fingerprint Challenge Dry Run. NIST Technical Note 2002, July 2018, } \\
\text { https://doi.org/10.6028/NIST.TN.2002 }\end{array}$ \\
\hline PEARSON & $\begin{array}{l}\text { Karl Pearson (20 June 1895) "Notes on regression and inheritance in the case of two } \\
\text { parents," Proceedings of the Royal Society of London, 58: } 240-242\end{array}$ \\
\hline \multirow[t]{3}{*}{ SAMPAT } & $\begin{array}{l}\text { M. P. Sampat, Z. Wang, S. Gupta, A. C. Bovik and M. K. Markey, "Complex Wavelet } \\
\text { Structural Similarity: A New Image Similarity Index," in IEEE Transactions on Image } \\
\text { Processing, vol. 18, no. 11, pp. 2385-2401, Nov. 2009. doi: 10.1109/TIP.2009.2025923 }\end{array}$ \\
\hline & $\begin{array}{l}\text { Code at http://freesourcecode.net/matlabprojects/62565/complex-wavelet-structural- } \\
\text { similarity-index-\%28cw-ssim\%29-in-matlab\#.WWzXKYTytGE }\end{array}$ \\
\hline & Requires matlabpyrtools from http://www.cns.nyu.edu/ lcv/software.php \\
\hline THAI & $\begin{array}{l}\text { Thai, Raymond, Fingerprint Image Enhancement and Minutiae Extraction, Student Report } \\
\text { submitted in partial fulfillment of requirements for the Honours Programme of the School } \\
\text { of Computer Science and Software Engineering, The University of Western Australia, } \\
\text { 2003, https://www.peterkovesi.com/studentprojects/raymondthai/RaymondThai.pdf, } \\
\text { (Retrieved 06/18/2018 }\end{array}$ \\
\hline WMC & $\begin{array}{l}\text { WikiMedia Commons, Boxplot vs. PDF, } \\
\text { https://commons.wikimedia.org/wiki/File:Boxplot_vs_PDF.svg; (Retrieved 06/18/2018). }\end{array}$ \\
\hline ZHANG & of noisy images." IEEE 1998. \\
\hline
\end{tabular}




\section{Appendix A Minutiae Extraction and Correspondence}

\subsection{Background}

A third-party, state of the art, fingerprint matcher software application was used to support the automated selection of registration candidates.

The minutiae extraction piece generates a text file containing minutiae $\mathrm{x}$-y coordinates, angle and "quality." The matcher piece generates a text file containing the list of corresponding minutiae.

\section{$9.2 \quad$ Image Data}

Rendered fingerprint images used for this guidance originate from two, general, device types: Contact and Contactless.

For the match-scenario process, a contact device was used as the exemplar (the gallery) while contactless or other contact devices were used as the query (the probe).

\subsubsection{Contact Device Image Data}

Four-finger slaps from right and left hands were captured and segmented into individual images corresponding to FGPs 02, 03, 04, 05, 07, 08, 09, and 10. All images are maintained as 500ppi, 8 bits per pixel depth.

Slap image segmentation coordinates were generated using proprietary software. Using the segmentation coordinates, fingerprints (images) were extracted/cropped into individual images.

\subsubsection{Contactless Device Image Data}

All contactless devices used in this study operate by taking a photo of a four-finger slap, performing the segmentation, and rendering the individual fingerprint segments into individual image files in BMP or PNG format. Therefore, there was no requirement to segment slaps as was the case for contact devices.

\subsection{Registration Candidates}

The goal of this process is to generate a list of "registration candidates" from corresponding minutiae for "known mate" match-pairs. A "match-pair" is a set of two fingerprint images that are known to correspond, i.e. same subject, same finger position.

We made use of a proprietary fingerprint matcher that had been modified to output lists of minutiae determined by the matcher to correspond to one another across the pair of fingerprint images input to the matcher. Thus, in addition to a match (or similarity) score, the tool yielded pairs of feature points from the image-pair that are used as "control points" by which to transform one image of the pair to overlay the other in a "best-fit" sense. The automated registration algorithm employed requires at least two sets (pairs) of corresponding feature points to compute the affine transformation to bring one image into registration with the other. Hence, image pairs that for which the tool failed to yield at least two sets corresponding minutiae were excluded from the registration process and from measurements requiring region correspondence. 


\subsubsection{Fingerprint Minutiae Extraction}

For each individual fingerprint image for the contact and contactless devices, minutiae features were extracted into an individual template file. The template was "decoded" into a text-file representation of the extracted minutiae to include $x$ - and $y$-coordinates data, feature angle, and quality. For this registration candidates process, only the coordinate data were utilized (angle and quality were ignored).

\subsubsection{Matcher}

For the matching process, the contact device was used as the exemplar (the gallery) while contactless and other contact devices were used as the query (the probe).

Match scenarios for known mates for various device comparisons were configured. In addition to a match score, the modified matcher, we refer to as the Minutiae Correspondence Tool yielded lists of minutiae pairs to serve as registration candidates. 


\section{Appendix B Matcher Testing}

This appendix describes the scoring methodology employed when determining the False Match Rate (FMR), False Non-Match Rate (FNMR), and consequent True Match Rate (TMR) from the match scores produced by the matcher; it also describes the specific circumstances of producing these match scores.

The matcher was used with a gallery of operational ten-print rolled records of nominally 3000051 subjects, chosen randomly from the Class C dataset used in the 2014 Fingerprint Vendor Test and Evaluation [NIST4]; We here refer to this gallery as the '3Million' gallery.

The matcher is most often used with multi-finger searches, as are employed when searching for a subject whose images are submitted in the format of an FD-249 Criminal or FD-258 Applicant Card. However, it is also capable of performing single-finger searches, as done in this experiment.

Because the 3-Million gallery is deliberately composed of operational data, the actual identity of subjects in this gallery is not known. In the past, we have detected some consolidation issues where a test probe fingerprint is matched to what is assumed to be a known non-matching print in the background data, appearing as a False Match. For this reason, we continued the practice which we have begun to employ when using operational data to determine FMR: we mirror the image of each finger in the probe set via a left-to-right transform, and assign it the equivalent finger position of the opposite [i.e., mirrored] hand. For example, if the original image was of the left thumb $(\mathrm{FGP}=6)$, we mirror it, and submit it to the matcher as an image of the right thumb (FGP=1).

The test included 60 subjects, all but two subjects yielding prints from four fingers of the right hand, with some failures to enroll (FTE) some prints. Hence, there were potentially $232[4 *(60-2)]$ fingers available to generate similarity scores from both TA and device TB, and 112 [4* (30 - 2)] fingers available from device TBrp. The probes from device TA produced 230 sets of similarity scores, a deficiency of two FTEs; the probes from device TB produced 225 sets, a deficiency of seven FTEs; and the probes from device TBrp produces 110 sets, a deficiency of two FTEs. There were 240 sets of similarity scores from C1, and thus no FTEs.

When computing scores, the gallery was treated as if its actual contents were the 3Million gallery augmented by the four-finger subject records from C1a. Similarly, when computing the scores for a device, only the results for the probe images from that device were considered. We did not consider the FTE's in the scoring for this preliminary examination.

The scoring rubric is described as follows: 
First, compute the total number of comparisons ${ }^{26}$ actually under consideration:

Total Comparisons $=$ size of probe set $*$ number of entries in repository

Total Mated Comparisons = summation across probe set of mates in

gallery for that probe

Total Unmated Comparisons $=$ Total Comparisons - Total Mated Comparisons

Second, at each possible similarity score, or threshold, determine:

True Mates: Number of mated comparisons whose score equals or exceeds threshold

False Mates: Number of non-mate comparisons whose score equals or exceeds threshold

Finally, at each possible threshold, compute:

TMR $=$ True Mates $/$ Total Mate Comparisons

FMR $=$ False Mates $/$ Total Non-Mate Comparisons

Note that the results of almost all comparisons, unless the gallery is quite small, are not reported; such comparisons can be said to have returned an implicit score of (or close to) zero. However, it is not necessary to generate all these scores. They are fully accounted for in the rubric described.

${ }^{26}$ Comparison $==$ one record of probe set evaluated by the matcher for similarity to one record in the repository. 


\section{Appendix C - Fabrication of Test Artifacts}

This section describes the design and fabrication of the Generation 8, 9, and $10^{27}$ Contactless Acquisition Test Artifact Suite, including material, dimensions and test pattern specifications. The Test Suite consists of multiple Generation 8 type fingerprint pattern artifacts together with the 3D Ronchi targets included as part of the Generation 8 artifact suite. Generation 10 consists of laser engravings from fingerprints of four subjects verified to be deceased.

\subsection{Material Specification and Artifact Dimensions}

The Generation 8, 9, and 10 Artifacts are constructed from a 1" $(0.394 \mathrm{~cm})$ diameter cast acrylic opaque tan rod. The rod is machined into a $60 \mathrm{~mm}$ circumference $(19.1 \mathrm{~mm}$ $(0.7519$ ") diameter, or $9.55 \mathrm{~mm}(0.376$ ") radius), with a finished length of $117.7 \mathrm{~mm}$ (4.63"). The finished length includes a $9.55 \mathrm{~mm}$ radius hemispherical dome at one end, a $15 \mathrm{~mm}$ threaded stud at the opposite end, and a $93.15 \mathrm{~mm}$ length between the former and latter, totaling the finished length of $117.7 \mathrm{~mm}$. This constitutes the base (or "blank") for engraving a fingerprint or geometric test pattern. See Section 11.5 for a full design schematic.

The color selection was intentional as experimentation revealed color to be important for some contactless devices. Very dark or very light materials led to poor sensor response for most of the devices tested. The tan color was one of several that yielded a reasonable distribution of gray levels. At least one device failed to acquire the target, or even experienced a catastrophic software error for colors towards the extremes of light and dark.

\subsection{Test Pattern Specification}

For Generation 8 two Ronchi ruling patterns were selected, one in an axial orientation and the other in a radial orientation. Both Ronchi test patterns are 1 cycle-per-millimeter frequency parallel bar patterns, similar to those specified in the PIV but with a 3D ridgevalley relief structure in place of the alternating black and white/clear bars in the PIV Geometric Accuracy target specification. The ridge of the 3D Ronchi pattern is the outer surface of the artifact and the valley of the Ronchi pattern is the portion of the pattern engraved into the artifact. In addition, instead of covering the entire capture area of a device with a flat material as specified in the PIV, the 3D Ronchi pattern wraps around the circumference of the rod. One hundred-eighty (180) degrees around the rod approximates "nail-to-nail" coverage and results in approximately 30 cycles of the pattern within a $30 \mathrm{~mm}$ span.

Specification for the engraved bars is $0.5 \mathrm{~mm}$ wide at a depth of $70 \mu \mathrm{m}$. Specification for the spaces between the $0.5 \mathrm{~mm}$ engraved bars is also $0.5 \mathrm{~mm}$ in width. The axial Ronchi pattern has the bars directed parallel to the long axis of the rod and extended to the tip of the hemispherical end. The radial Ronchi pattern, on a separate target, has the bars directed perpendicular to the long axis of the rod and extending partially (roughly halfway) onto the hemispherical end. In the case of variation in the ridge or valley width from the $0.5 \mathrm{~mm}$

${ }^{27}$ Early work in this area led to the fabrication of generations 1 through 7, which are now considered obsolete. Only generations 8 and above are presented in this document. 
specification, preservation of the $1 \mathrm{~mm}$ cycle dimension has been prioritized. The engraving has vertical side walls and a flat bottom. The Ronchi pattern covers slightly more than 180 degrees around the target to allow for reasonable marginal variance in presentation positioning with respect to a device under test. A single straight-line parallel to the long axis and 180 degrees from the center of the overall engraved pattern is included to provide assistance in centering the pattern for presentation to the device sensor, where the reference line will be centered atop for viewing and opposite the center of the pattern directed toward the device sensor.

The Generation 8 Ronchi target, pictured in Figure 32 has been built on established Ronchi test pattern standards specifications for testing fingerprint acquisition devices [EBTS] but extends to 3D measurement of output distortion, uniformity, across bar and resolution accuracy in both vertical and horizontal directions.

The Generation 9 Fingerprint target is intended to closely replicate the features of a real fingerprint pattern, using the actual fingerprint of a deceased subject from a newly compiled dataset [NIST5] (see Figure 37) The fingerprint pattern is processed for laser engraving by thresholding and converting the image to a binary (black and white only) colorspace, mirroring the image horizontally in order to provide a negative of the impression that replicates the orientation of the pattern on the finger which left the impression, and then inverting the colors in order to provide the laser engraver with an image where black pixels indicate where the laser should be fired to engrave the material (see Figure 38).

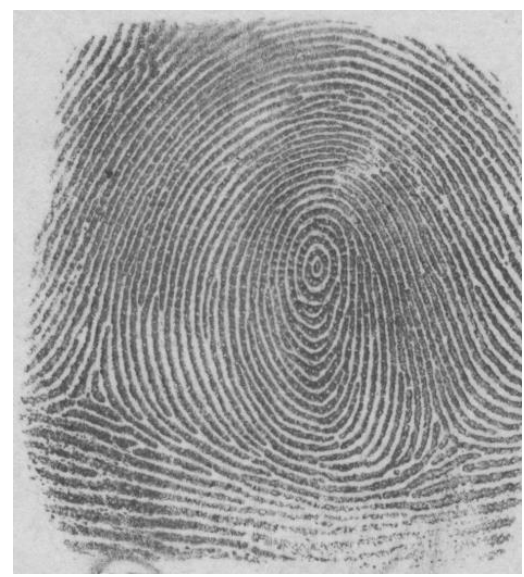

Figure 37 - Original Fingerprint Impression Image

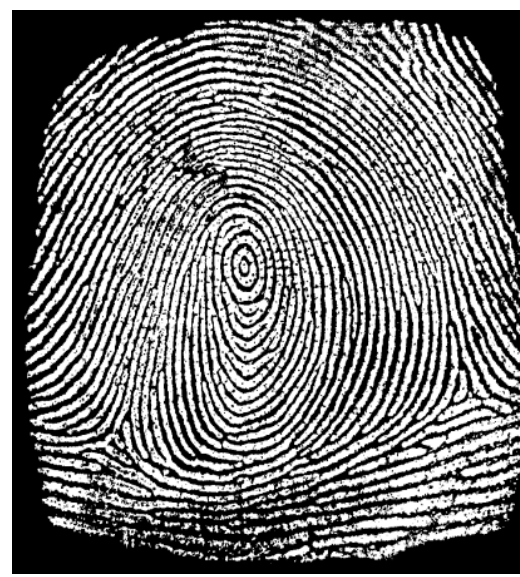

Figure 38 - Fingerprint Image Processed for Laser Engraving

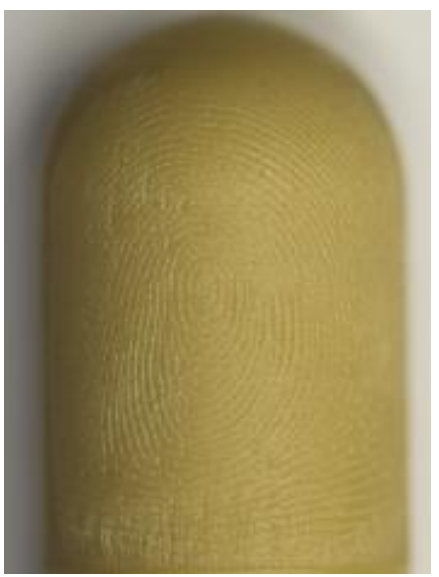

Figure 39 - LaserEngraved Fingerprint Target

The Generation 10 concentric circle target was specifically designed to allow for the detection of potential anomalies in the reproduction of topographic features when captured with contactless devices. This pattern consists of a set of concentric circles (shown in 
Figure 40), and was designed to reveal inconsistencies in the polarity or contrast reversal of the resulting image as well as other potential geometric distortions.

As with the Generation 9 Fingerprint target patterns, the Generation 10 concentric circle target pattern has been prepared for laser-engraving with black areas indicating where the laser should be fired to engrave the pattern into the surface of the blank target. As such, this results in finished targets with engraved areas of $\sim 70 \mu \mathrm{m}$ in place of the black areas of the patterns depicted in the figures above. Photos of the finished target is shown below in Figure 41.

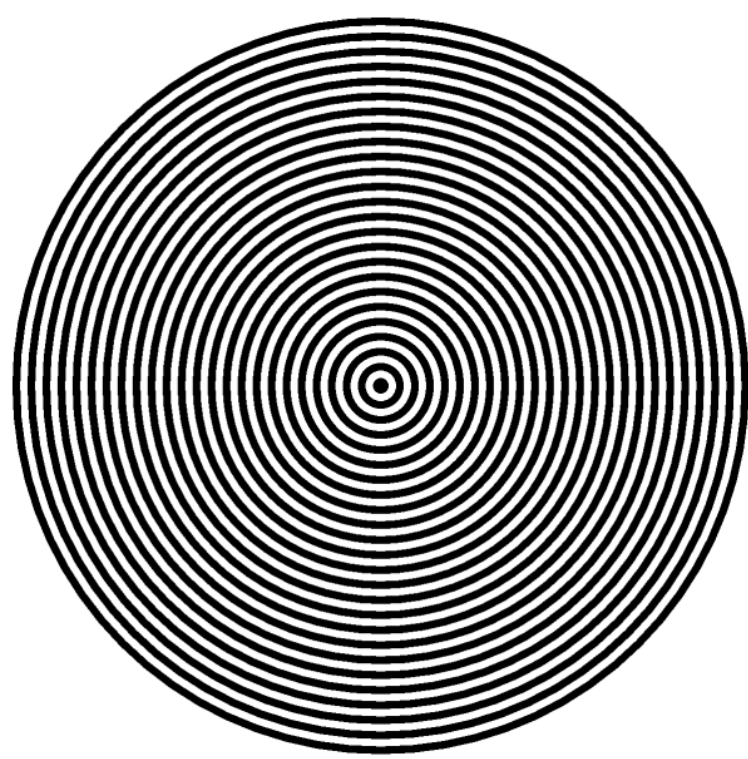

Figure 40 - Concentric Circle Target Pattern

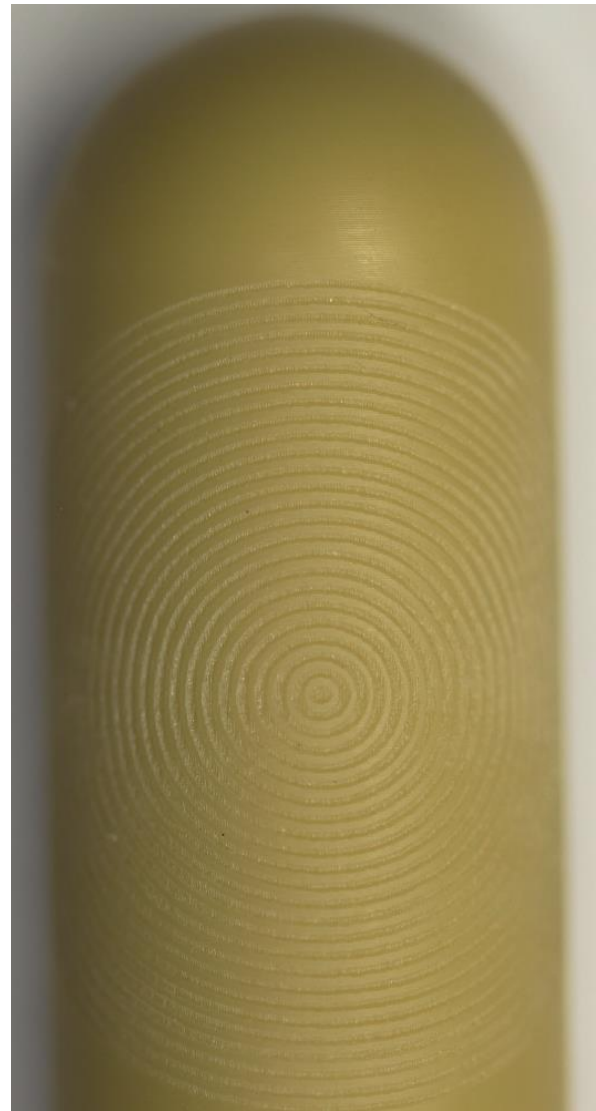

Figure 41 - Laser-Engraved Concentric Circle Target

The concentric circle target, as depicted in Figure 40 consists of 25 concentric circles with lines that are approximately $0.03(0.012 ") \mathrm{cm}$ thick, and spaced $0.03 \mathrm{~cm}(0.012 ")$ apart. The thickness and spacing of these lines is based on measurements observed in contactbased 1000 ppi fingerprint images from an existing fingerprint database. With laser engraving performed at $1000 \mathrm{ppi}$, this results in circles which approximate the thickness and spacing of fingerprint ridges but with the more predictable geometry of a perfect circle. 


\subsection{Target Identification}

Due to their independent respective fabrication processes, the Generation 8 Ronchi and Generation 9 Fingerprint series of targets feature slightly different styles of the serial numbers used to uniquely identify them. Two such examples are shown below in Figure 42 and Figure 43.

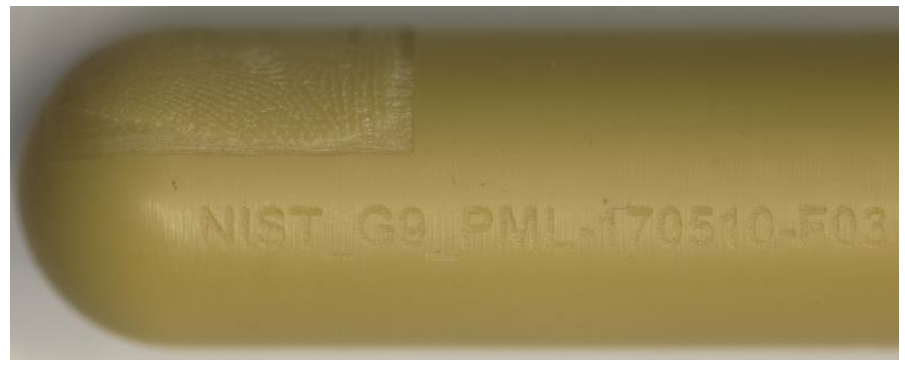

Figure 42 - Laser-Engraved Target Serial Number

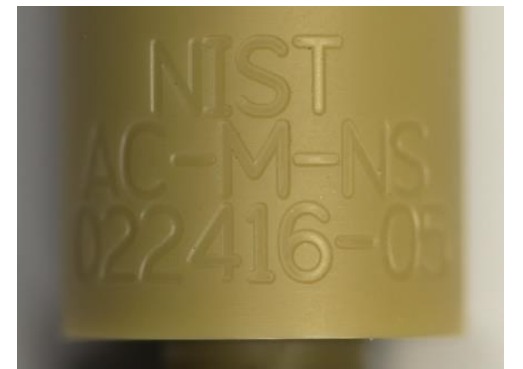

Figure 43 - Machined Target Serial Number

The laser-engraved fingerprint target is identified with the serial number NIST G9_PML170510-F03, located adjacent to the fingerprint pattern in small print (Figure 42). In this format, $G 9$ refers to the target pattern design generation (9), while $P M L$ refers to NIST's Physical Measurement Lab where the target was engraved, and 170510 refers to the date on which the target was engraved (May 10 ${ }^{\text {th }}, 2017$ ). The final token, F03 identifies the individual fingerprint pattern amongst the set of four fingerprint patterns that comprise a set of Generation 9 Fingerprint Targets.

The machined Ronchi target is identified with the serial number NIST AC-M-NS-02241605 , located towards the threaded end of the target. In this format, $A C$ refers to the material (acrylic) from which the target was fabricated, while NS refers to the NIST (machine) shop, and 022416 refers to the date on which the target was machined (February $24^{\text {th }}, 2016$ ). The final token, 05 refers to the sequence number of this particular target in a series of similar targets machined on the same date.

These slightly different styles of serial numbers are featured on the most current iterations of the various target designs due to their independent development processes, plans for future generations include a common serial number format and location for all target designs. 


\subsection{Measurements}

Physical measurements of both the axial and radial Ronchi targets were made using a Zygo NewView 7300 3D optical surface profilometer, which is capable of measurements in all three physical dimensions, as well as a Wild M7 zoom stereomicroscope (lab microscope) fitted with Martin Microscope SLR adapter connected to a Canon EOS 5D DSLR camera, which is only capable of measuring in two dimensions (width and height).

Measurements made with the optical profilometer began by masking and sputter-coating the targets with gold using a Denton Vacuum Desk V HP deposition system for $120 \mathrm{~s}$ at 75 $\mathrm{mA}$. This results in a thin $(\sim 10 \mathrm{~nm})$ layer of gold deposition for enhanced light reflection. The coated samples were then imaged with optical profilometer at magnifications of $5 \times$ and 10x. Images were analyzed for groove pitch and depth using the MetroPro film analysis software provided with the profiler.

Photos of the targets taken using the lab microscope were captured in the same frame as a metric ruler for proper scaling. The GNU Image Manipulation Program (GIMP) was used to view the images and measure the number of pixels occupied by $1 \mathrm{~mm}$ as shown on the metric ruler and this value was then used to calculate the physical size of features on the target, based on further measurements of features in pixels using GIMP. Averaged values calculated based on several samples using both methods are shown in Table 5 below.

Table 5 - Ronchi Target Physical Measurements

\begin{tabular}{|c|c|c|c|c|c|}
\hline $\begin{array}{l}\text { Ronchi } \\
\text { Pattern/Target }\end{array}$ & Measurement & $\begin{array}{l}\text { Average } \\
\text { Width } \\
\text { (Ridge) }\end{array}$ & $\begin{array}{l}\text { Average } \\
\text { (Valley) }\end{array}$ & Width & $\begin{array}{l}\text { Average } \\
\text { Depth } \\
\text { (Valley) }\end{array}$ \\
\hline Radial & $\begin{array}{l}\text { Optical } \\
\text { Profilometer }\end{array}$ & $500 \mu \mathrm{m}$ & $500 \mu \mathrm{m}$ & & $50 \mu \mathrm{m}$ \\
\hline Radial & Lab Microscope & $480 \mu \mathrm{m}$ & $510 \mu \mathrm{m}$ & & $N / A^{28}$ \\
\hline Axial & $\begin{array}{l}\text { Optical } \\
\text { Profilometer }\end{array}$ & $520 \mu \mathrm{m}$ & $480 \mu \mathrm{m}$ & & $67 \mu \mathrm{m}$ \\
\hline Axial & Lab Microscope & $490 \mu \mathrm{m}$ & $500 \mu \mathrm{m}$ & & $N / A^{28}$ \\
\hline
\end{tabular}

During initial trials of the laser engraving process, the laser engraver was calibrated to a depth of $70 \mu \mathrm{m}$ within a tolerance of $3 \%(+/-\sim 2 \mu \mathrm{m})$. Measurements of the ridge and valley widths taken using the lab microscope system described above showed near-perfect correlation with the known geometry of the fingerprint impression used to create the pattern.

\footnotetext{
${ }^{28}$ Note that the lab microscope system described above is not capable of measuring depth.
} 


\subsection{Target Design Schematic}

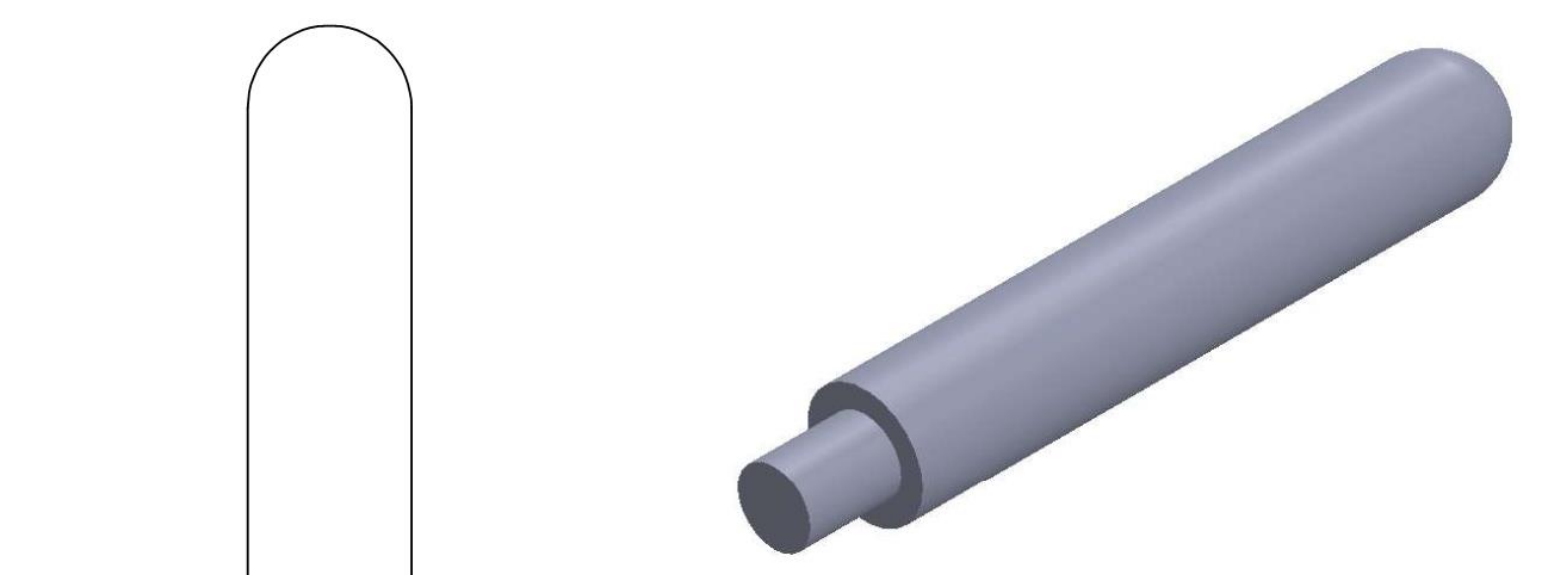

Threads are $\mathrm{M} 12.00 \times 1.75$

12.000 major diameter

10.274 minor diameter

Pitch $=1.75$

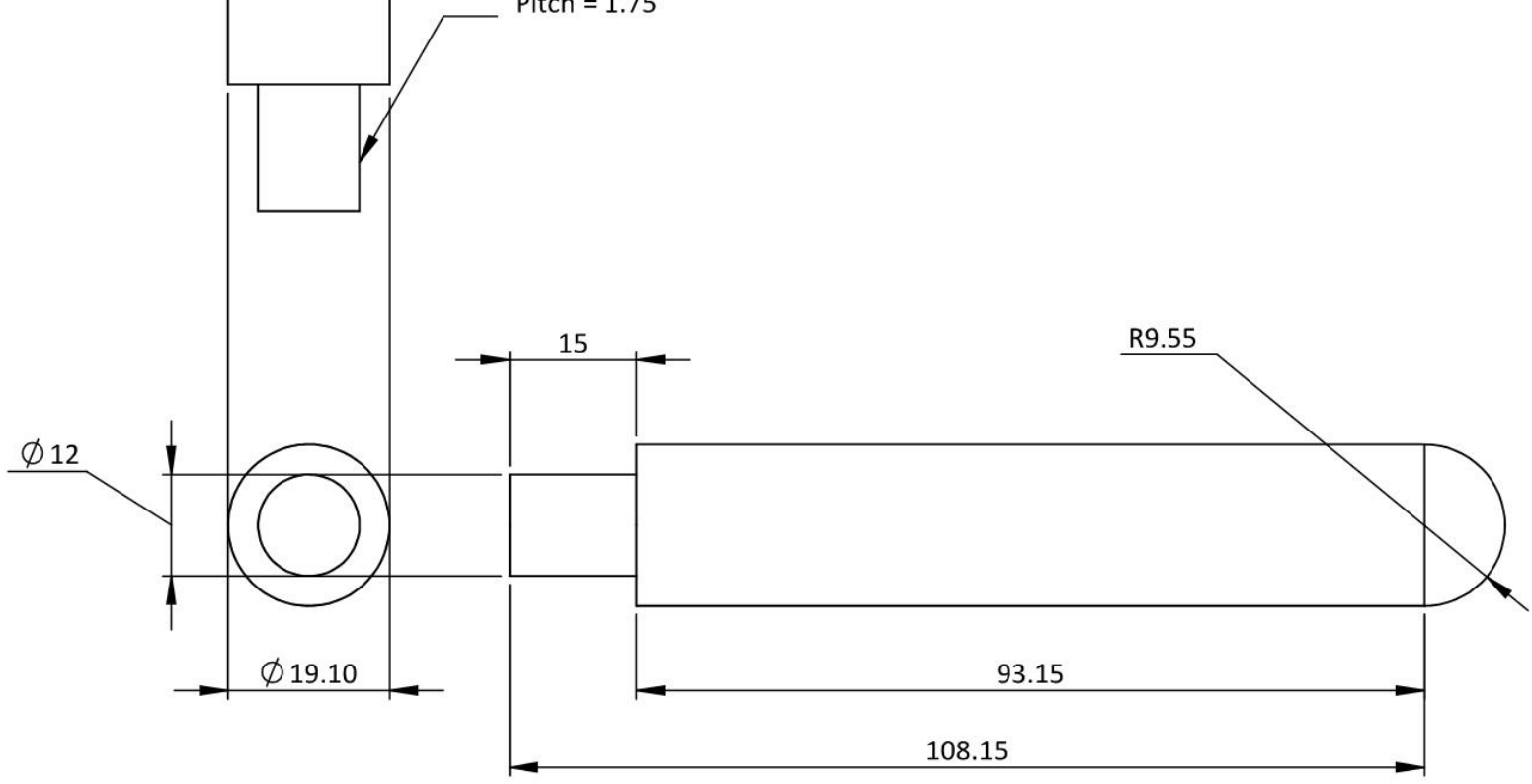

Figure 44 - Design schematic for fabrication of targets from 1" $(25.4 \mathrm{~cm})$ acrylic rods. Units shown in schematic are in centimeters $(\mathrm{cm})$. 